Adv. Studies Theor. Phys., Vol. 8, 2014, no. 7, 293 - 343

HIKARI Ltd, www.m-hikari.com

http://dx.doi.org/10.12988/astp.2014.4112

\title{
On the Unification of Higgs-Nambu Goldstone Boson in the Formation of Near-brane Exotic Scalar \\ via Scherk-Shwarz Breaking in 5-Dimensional \\ SU(6) Symmetry
}

\author{
F.P. Zen \\ Indonesian Center for Theoretical and Mathematical Physics \\ Theoretical Physics Laboratory, THEPI Research Division \\ Institut Teknologi Bandung,Jl. Ganesha 10, Bandung 40132, Indonesia
}

\section{A. Hartanto}

Indonesian Center for Theoretical and Mathematical Physics

\section{J.S. Kosasih}

Indonesian Center for Theoretical and Mathematical Physics

Theoretical Physics Laboratory, THEPI Research Division,

Institut Teknologi Bandung,Jl. Ganesha 10, Bandung 40132, Indonesia

Copyright (c) 2014 F.P. Zen, A. Hartanto and J.S. Kosasih. This is an open access article distributed under the Creative Commons Attribution License, which permits unrestricted use, distribution, and reproduction in any medium, provided the original work is properly cited.

\begin{abstract}
The 5D Scherk-Schwarz breaking with orbifold $S^{1} / Z_{2}$ in $\mathrm{SU}(6)$ symmetry under special condition leads to a dimensional deconstruction without gauge symmetry breaking where $\mathrm{SU}(6)$ Little Higgs-like scalars show up either as weakly-coupled or strongly-coupled. The latest, together with the broken fifth component of 5D gauge boson, composes Coleman-Weinberg potential which can be generalized by substituting PNB (Pseudo Nambu-Goldstone boson) Higgs as the broken fifth component, with Nambu-Goldstone boson being the third component of a massive 4D gauge boson in electroweak theory. A doublevacua symmetry emerges, based on Baker-Campbell-Hausdorff approach, with its shift and twist breaking terms which facilitate the unification of Higgs-NGB to become exotic scalars named as Scherk-Schwarz scalars. Under unitary transformation the double-vacua shift symmetry breaks via twistedly-broken manner
\end{abstract}


i.e. the NGB swallowing and digesting mode where the first consists of transferring 2 (two) degrees of freedom from 2 (two) NGBs without additional mass while the second with additional mass to PNB Higgs in the Higgs-NGB unification. The unification results in a free and bonded gauge-like single scalar which becomes the main components of exotic scalars such as 3-scalar Higgs, Higgs-like and gauge-like 3-component scalars. Ranges of masses for the exotic Higgs suit the latest LHC data with excluded zone $145-466 \mathrm{GeV}$ while for the exotic 3-component scalars run continuously up to $1.5 \mathrm{TeV}$.

Keywords: Orbifold, Scherk-Schwarz breaking, Little Higgs, Standard Model, Double vacua, Nambu-Goldstone boson, Scherk-Schwarz scalar

\section{Introduction}

GUT model based on $\mathrm{SU}(6)$ symmetry [1] suggests that the electroweak scale physics is realized via symmetry breaking $\mathrm{SU}(6) \rightarrow \mathrm{SU}(3)_{\mathrm{C}} \otimes \mathrm{SU}(3)_{\mathrm{H}} \otimes \mathrm{U}(1)_{\mathrm{C}}$ and subsequently to $\mathrm{SU}(3)_{\mathrm{H}} \rightarrow \mathrm{SU}(2)_{\mathrm{L}} \otimes \mathrm{U}(1)_{\mathrm{B}}$ but has a severe shortcoming, that is, no appropriate Higgs multiplet $[2,6,15]$.

Following recent development on extra dimension physics $[22,27,32,36,43]$, the Scherk-Schwarz breaking [3,19,20,22,27] and Orbifold breaking [12,22,23,25, 26,27] have opened the way for producing Higgs through the extra-dimensional compactification effect which induces the Higgs itself, and known as the gauge-Higgs boson unification $[11,12,21,22,27]$. Recently a grand gauge-Higgs unification based on 5D $\mathrm{SU}(6)$ compactified on an orbifold $S^{1} / Z_{2}$ with fermions in two 6-plets and one 20plet shows successful breaking with no proton decay at tree level but a little low compactification scale and heavy Higgs [8]. Other work such as Hosotani mechanism with non-zero VEV being developed by extra-dimensional parameters results in instant gauge symmetry breaking after dimensional deconstruction $[25,27,47]$ and some other works in extra dimension and non-Higgs mechanism have been developed $[3,4,13,14,15,21,22,24,26,27,35,40,41,48]$ including the pioneer one [32,34].

On the other hand Little Higgs mechanism, with pseudo Nambu-Goldstone boson (PNB) as Little Higgs, has been developed simultaneously. It provides massive PNB after shift symmetry breaking and becomes an alternative to Higgs breaking [9,2830,33,34,37-39,46].

Corresponding to 5D SU(6) local gauge symmetry, based on AdS/CFT correspondence, $\mathrm{SU}(6)$ global symmetry also exists [3,8], and breaks after shift symmetry breaking takes place, and produces massive PNB. It means that the scalars come from the fifth components of $5 \mathrm{D}$ gauge bosons $[8,11,12,25,27]$ and/or directly from the bulk $[13,26,27]$.

In the paper [3], special conditions of Scherk-Schwarz mechanism are utilized to resolve the problem of breaking the SU(6) GUT. The trivial and the non-trivial breaking pattern are simultaneously realized by compactification of orbifold $S^{1} / Z_{2}$ in 5-dimensional (5D) SU(6), not like the trivial the (pseudo) non-trivial condition generates the scalar bosons. The condition facilitates for the periodic 5D scalar $[3,11,12,13,26]$ with extra-dimensional local and global symmetry for small extra dimension in the so-called near-brane area $[3,8,35]$. Here, in the near-brane area, 
the first (trivial) symmetry breaking of $5 \mathrm{D} \mathrm{SU}(6) \rightarrow 4 \mathrm{D} \mathrm{SU}(6)$ is triggered by ScherkSchwarz mechanism and followed by trivial and pseudo non-trivial Orbifold breaking $[3,23,26]$ to produce $\mathrm{SU}(6)$-origin would-be Little (Baby) Higgs scalar as the origin of SU(6) will-be-SimplestLittleHiggs and SU(6) Baby Higgs scalars successively [3]. Trivial Orbifold Breaking (TOB) and Pseudo non-trivial Orbifold Breaking (POB) which facilitate dimensional deconstruction but still keep the symmetry intact, in principle, do produce 'exact' scalar boson, and the perturbative, incomplete series of exponential form, of strongly-coupled SU(6)-origin would-be Little (Baby) Higgses. It means that they decouple from and cannot exist in $\mathrm{SU}(6)$ and be transformed into $\mathrm{SU}(6)$ Little Higgs-like scalars which transform itselves again under triplet-triplet splitting $[3,48]$ and higher modes decoupling $[3,22,26]$.

In this paper, the presented material is organized as follows: the 5-dimensi onal near-brane model with SU(6) Little Higg-like scalars is discussed in the next section. Subsequently we discuss on the SU(6) 5D-twist Nambu-Goldstone boson (NGB) emerging in the generalized Coleman-Weinberg potential replacing the broken fifth component of $5 \mathrm{D}$ gauge boson or as a substitute for Higgs boson under gauge-Higgs unification principle. In view of NGB as the third component of a massive 4D gauge boson in electroweak theory and Higgs as Pseudo Nambu-Goldstone boson (PNB) in Little Higgs theory, NGB can emerge as the broken fifth component, instead of PNB (Higgs), with equal probability producing generalized Coleman-Weinberg potential where unification of Higgs-NGB becomes possible. This unification can only take place in a double-vacua system where twistedly-broken shift (symmetry) breaking happens. This is discussed in the section following the above where, based on BakerCampbell-Hausdorff formula, double-vacua shift and twist factors are introduced. The SU(3) and SU(6) unification of Higgs-NGB are discussed within the subsection of Scherk-Schwarz twist breaking where a double-vacua property plays an important role in producing 2 (two) basic types of the so-called Scherk-Schwarz scalars which are basically exotic scalars.

Next, we discuss the double-vacua shift symmetry breaking under unitary transformation from which one obtains shiftedly-and twistedly-broken Scherk-Schwarz scalars. Further sections discuss the basic properties of Higgs-NGB unification covering the transferring of exoticity property, after triplet-triplet splitting and swallowing-digesting mode, and its conservation under symmetry breaking. Finally, a brief discussion is given for the exotic 3-scalar Higgs, Higgs-like and gauge-like 3component scalars, its relevant mass-squared and related phenomenological aspects.

\section{The 5D Near-brane Model with SU(6) Scalar}

\subsection{The Scherk-Schwarz and Orbifold breaking}

First of all let us consider the orbifold breaking in 5D SU(6) compactified in $\mathcal{M}^{4} \times$ $S^{1} / Z_{2}$. Before discussing the details, a brief review on Scherk-Schwarz mechanism on orbifold $S^{1} / Z_{2}$ is given below.

The invariance of a theory compactified on 5-dimensional space, $\mathcal{M}^{4} \times S^{1} / Z_{2}$, demands $\mathcal{L}_{5}[\phi(x, y)]=\mathcal{L}_{5}\left[\phi\left(x, \tau_{g}(y)\right)\right] . \quad$ The ordinary compactification satisfies 
$\phi\left(x, \tau_{g}(y)\right)=\phi(x, y)$ which is a special case of general Scherk-Schwarz compactification condition $\phi\left(x, \tau_{g}(y)\right)=T_{g} \phi(x, y)[19,20,23,27]$. Here, $\tau_{g}(y)$ is the mapping operator for $y$, and $T_{g}$ is the twist transformation operator.

In general, orbifold compactifications have similar principles. The condition is written as $\phi\left(x, \zeta_{2}(y)\right)=Z_{2} \phi(x, y)$. The operator $T_{g}$ should satisfy the so-called consistency condition as below, anyway,

$$
T_{g} Z_{2} T_{g}=Z_{2}, \quad T_{g}=e^{2 i \pi \vec{\beta} \cdot \vec{\lambda}}=e^{2 i \pi \omega Q},
$$

where $\lambda^{a^{\prime}}$ are the hermitian generators and $Q$ is the generator with a predefined direction in generator space, while $\omega$ and $\beta^{a^{\prime}}$ are the corresponding parameters. Combining with the above consistency condition and expanding infinitesimally one immediately finds the condition $[23,27]$,

$$
\left\{\vec{\beta} \cdot \vec{\lambda}, Z_{2}\right\}=0 \quad \text { and } \quad\left[T_{g}, Z_{2}\right]=0 .
$$

These relations determine the broken and unbroken parts of the generators under consideration. The latter also gives the singular solution $T_{g}= \pm 1$.

For 5D theory compactified on the $S^{1} / Z_{2}$ orbifold with the Scherk-Schwarz twist as in Eq. (1), the twisted field obeys,

$$
\phi(x, y+2 \pi R)=e^{2 i \pi \omega Q} \phi(x, y),
$$

where $R$ is the compactification radius. Symmetry breaking is achieved if the symmetry generated by $Q$ is broken and $Q^{\prime}(Q)$ satisfies the (anti)commutative relation in Eq. (2), that is

$$
\left\{\omega Q, Z_{2}\right\}=\omega\left\{Q, Z_{2}\right\}=0, \quad\left[Q^{\prime}, Z_{2}\right]=0 .
$$

Now we are ready to apply the preceding discussion on the $S^{1} / Z_{2}$ orbifold to $\mathrm{SU}(6) . \quad Z_{2}$ for $\mathrm{SU}(6)$ can be constructed based on 3 arrays of $\mathrm{SU}(2)$-type matrix along its diagonal elements consisting of $Z_{2}^{(0)}$ and $Z_{2}^{(1)}$ following orbifold singular points as follows,

$$
Z_{2}^{(0)}=Z_{2}^{(1)}=\left(\begin{array}{cccccc}
1 & 0 & & & & \\
0 & 1 & & & & \\
& & 1 & 0 & & \\
& & 0 & -1 & & \\
& & & & -1 & 0 \\
& & & & 0 & -1
\end{array}\right), Z_{2}^{(0)} Z_{2}^{(1)}=I_{6}=U,
$$

this form satisfies the boundary conditions of $S^{1} / Z_{2}$ orbifold suitable to realize the symmetry breaking $\mathrm{SU}(6) \rightarrow \mathrm{SU}(3) \otimes \mathrm{SU}(3) \otimes \mathrm{U}(1)$ in the non-trivial pattern [3,23,27].

Let us adopt the special condition in Eq. (4) through setting $Z_{2}=U$ for commutator and $Z_{2}=Z_{2}^{(0)}=Z_{2}^{(1)}$ for anti-commutator due to $Q^{\prime}=$ all $\mathrm{SU}(6)$ generators $=\lambda_{a^{\prime}}, a^{\prime}=1,2, \ldots, 35$ and $Q=0[11,12,23,27]$, for the current $5 \mathrm{D} \rightarrow 4 \mathrm{D}$ case of $\mathrm{SU}(6)$, namely trivial and pseudo non-trivial patterns and write as $5 \mathrm{D}$ $\mathrm{SU}(6) \rightarrow 4 \mathrm{D} \mathrm{SU}(6)$ or $5 \mathrm{D}(y \sim 0) \mathrm{SU}(6)$ from which a near-brane is to be defined later. In the near-brane one has the further breaking as $4 \mathrm{D} \mathrm{SU}(6) \rightarrow 4 \mathrm{DSU}(3) \times$ $\mathrm{SU}(3) \times \mathrm{U}(1)$ where $4 \mathrm{D}$ here is equivalent to $5 \mathrm{D}(y \sim 0)$ and $\mathrm{SU}(3) \times \mathrm{SU}(3) \times \mathrm{U}(1)$ with $y=0$ is brane itself. 


\subsection{Near-brane Coleman-Weinberg potential and global-local gauge correspondence}

We adopt the 5D-model in near-brane where extra dimension $y \sim 0$ and $\mathrm{SU}(6)$ global (local) gauge symmetry $[7,8]$ exist (to be explained) with the $4 \mathrm{D}$ particles living in the 2 branes and 5D gauge bosons as well as scalar bosons in the bulk. One brane corresponds to fixed point $y=0$ and the other brane corresponds to another fixed point $y=\pi R$ of the $S^{1} / Z_{2}$ orbifold.

The Lagrangian can be written accordingly as

$$
\mathcal{L}_{5}^{\mathrm{SU}(6)}=D^{M} \Phi^{\dagger} D_{M} \Phi, \quad M=(\mu, y),
$$

where $\Phi=\left(\Phi_{1}, \Phi_{2}, \Phi_{3}, \Phi_{4}, \Phi_{5}, \Phi_{6}\right)^{T} \equiv\left[\Phi_{k}\right], k=1,2, \cdots, 6$ is scalar boson in the fundamental representation of $\mathrm{SU}(6)$, and scalar field $\Phi$ is expressed as periodic scalar field $\widetilde{\Phi}$ via the following relationship [6]

$$
\Phi(x, y)=e^{i \omega Q_{v} y / R} \widetilde{\Phi}(x, y)=e^{i Q_{v} \alpha} \widetilde{\Phi}(x, y),
$$

where global (local) gauge parameter $\alpha$ is obtained as

$$
\alpha=\frac{\omega y}{R}
$$

wEq. (7) can be obtained as solution of Eq. (3), $\alpha$ turns out to be a global gauge phase factor for $\alpha \ll 1$ and local gauge in the limited space for $\alpha<1$, hence, to be discussed further [3,7] later, and $Q_{v}$ represents $\mathrm{SU}(6)$ broken generators in the direction of $\mathrm{VEV}$ s $[3,11,12,27]$. Defining $D^{\mu}\left(D_{\mu}\right)$ as $4 \mathrm{D}$-covariant derivative and $D^{y}\left(D_{y}\right)$ as fifth-dimensional covariant derivative with $T^{a}=\lambda^{a} / 2\left(=T_{a}\right)$, and $g_{5}$ is the $5 \mathrm{D}$ coupling constant

$$
\begin{array}{ll}
D_{\mu}=\partial_{\mu}-i g_{5} A_{\mu}^{a} T_{a}, & D^{\mu}=\partial^{\mu}+i g_{5} A_{a}^{\mu} T^{a} \quad \text { and } \\
D_{y}=\partial_{y}+i g_{5} A_{y}^{a} T_{a}, & D^{y}=\partial^{y}-i g_{5} A_{a}^{y} T^{a},
\end{array}
$$

one can separate the $4 \mathrm{D}$-brane from the Lagrangian $\mathcal{L}_{5}^{\mathrm{SU}(6)}=\mathcal{L}_{\mu}^{\text {brane }}+\mathcal{L}_{(\theta y), y}^{\text {near-brane }}+$ $\mathcal{L}_{y}^{\mathrm{SU}(6)}$, where $4 \mathrm{D}$ near-brane is just in-between the brane and the bulk.

In the brane $y=0$ and near-brane $y \sim 0$ the even scalar bosons in Eq. (7) becomes as $\widetilde{\Phi}^{(i)}(x)=\left.\widetilde{\Phi}^{(i)}(x, y)\right|_{y=0 \text { or } ~ 0}, i=1,2$.

For the near-brane space Neumann boundary condition dictates $D^{y} \widetilde{\Phi}^{\dagger}=D_{y} \widetilde{\Phi}=$ 0 and $D^{\mu} \widetilde{\Phi}^{\dagger}=D_{\mu} \widetilde{\Phi}=0$ while Lorentz invariance requires the elimination of Lorentzviolating terms which make the only near-brane equation with $\delta_{c}(y) \sim 1$ for $y \sim 0$ and $Q_{v}=0$, as follows [3],

$$
\mathcal{L}_{y}^{\text {near-brane }}=\frac{1}{2} g_{5}^{2}\left(\widetilde{\Phi}^{(i) \dagger} A_{a}^{y}\right)\left(A_{y}^{a} \widetilde{\Phi}^{(i)}\right)
$$

where $\delta_{c}(y)$ is defined as Gaussian-like distribution function with the crest width $y \sim 0$ so that $\delta_{c}(y)=1$. Now, one has $\widetilde{\Phi}^{(i)}=\widetilde{\Phi}_{+}^{(i)}(x), \widetilde{\Phi}^{(i) \dagger}=\widetilde{\Phi}_{+}^{(i) \dagger}(x)$, with $i=1,2$, 
and $(+)$ denoting even parity. In this near-brane bulk ( $y$-area), one has the subsets (sextet out of $2 \times 9$ broken $A_{\hat{a}}^{y}$ and $A_{y}^{\hat{a}}$ ) as

$$
A_{\hat{a}}^{y} T^{\hat{a}} \supset \widetilde{\Phi}^{(j)}, \quad A_{y}^{\hat{a}} T_{\hat{a}} \supset \widetilde{\Phi}^{(j) \dagger},
$$

which is based on gauge-Higgs unification principle, where $\widetilde{\Phi}^{(j)}\left(\right.$ or $\left.\widetilde{\Phi}^{(j) \dagger}\right)$ is diagonal $3 \times 3$ sub-matrix component of $6 \times 6$ matrix of $A_{\hat{a}}^{y} T^{\hat{a}}$ (or $A_{y}^{\hat{a}} T_{\hat{a}}$ ) and $j=1,2$ as in Eq. (11). Consequently Eq. (10) can be rewritten, using $\widetilde{\Phi}^{(j)}$ as diagonal component of $A_{\hat{a}}^{y} T^{\hat{a}}$, and $\lambda_{y}^{(6)}=g_{5}^{2}$, as [3],

$$
V_{y}^{(6)}=\lambda_{y}^{(6)}\left(\widetilde{\Phi}^{(i) \dagger} \widetilde{\Phi}^{(j)}\right)\left(\widetilde{\Phi}^{(j) \dagger} \widetilde{\Phi}^{(i)}\right) .
$$

In order to determine $\widetilde{\Phi}^{(i)}$ and $\widetilde{\Phi}^{(j)}$ which are not necessarily the same but it must be corresponding one to another due to existing correspondence between 5D local gauge and 4D global gauge symmetry as shown later on, then Eq. (7) can be written, applying $y \sim 0$ for periodic scalar under global (local) gauge transformation $e^{i Q_{v} \alpha}$, as $\Phi(x, 0) \sim e^{i Q_{v} \alpha} \widetilde{\Phi}(x)$ [3], where global gauge is defined as, Eq. (8), with $\omega$ is Scherk-Schwarz parameter. To start our analysis some parameters must be defined accordingly.

At the orbifold singular points which are $4 \mathrm{D}$ and have the twist factor $T_{g}=+1$ for $y=0$ and $T_{g}=-1$ for $y=\pi R$, one can assign without loss of generality, two non-zero $\mathrm{VEV}$ s at one fixed point such as [3],

$$
v=\frac{1}{\sqrt{\pi R}}\left(\begin{array}{c}
0 \\
0 \\
f_{1} \\
0 \\
0 \\
0
\end{array}\right), v^{\prime}=\frac{1}{\sqrt{\pi R}}\left(\begin{array}{c}
0 \\
0 \\
0 \\
0 \\
0 \\
f_{2}
\end{array}\right) \text {, for } y=0 \text { and } y \sim 0 .
$$

The parametrization of SU(6) would-be Baby (Little) Higgs is governed by the number of scalar doublets which are allowed to be put in $6 \times 6$ matrix. Thus it depends on the number of the generated NGBs through the condition [3,43],

$$
\lambda_{a^{\prime}}\left\langle\widetilde{\Phi}_{k}\right\rangle \neq 0, \quad\langle\widetilde{\Phi}\rangle_{01}=v, \quad\langle\widetilde{\Phi}\rangle_{02}=v^{\prime}
$$

with $\lambda_{a^{\prime}}=\lambda_{1}, \lambda_{2}, \ldots, \lambda_{35}$.

Eq. (14) gives 22 free NGBs in total. Finally one has 8 scalar bosons including PNBs which create 4 scalar doublets to be assigned as the SU(6) would-be Baby (Little) Higgs as follows [3],

$$
\theta=\frac{1}{f}\left(\begin{array}{cc}
(0)_{3 \times 3} & \left(\begin{array}{cc}
(0)_{2 \times 2} & (h)_{2 \times 1} \\
\left(h^{\prime \dagger}\right)_{1 \times 2} & 0
\end{array}\right) \\
\left(\begin{array}{cc}
(0)_{2 \times 2} & \left(h^{\prime}\right)_{2 \times 1} \\
\left(h^{\dagger}\right)_{1 \times 2} & 0
\end{array}\right) & (0)_{3 \times 3}
\end{array}\right),
$$

where $f^{2}=f_{1}^{2}+f_{2}^{2}$. The scalar doublets $h$ and $h^{\prime}$ are the would-be SM Higgs as will be explained later. 


\subsection{SU(6) LittleHiggs-like scalar}

\subsubsection{SU(6) would-be Baby (Little) Higgs}

Let's start with an extra-dimensional scalar from the near-brane corresponding to $5 \mathrm{D}(y \sim 0)$ gauge boson of which one takes Kaluza-Klein $(\mathrm{KK})$ expansion with even and odd parities, one has the even fields [15],

$$
\widetilde{\Phi}_{+}^{(i)}(x)=\frac{1}{\sqrt{\pi R}}\left[\widetilde{\Phi}_{+}^{0}(x)\right]_{i}+\sqrt{\frac{1}{\pi R}} \sum_{n=2}^{\infty}\left[\widetilde{\Phi}_{+}^{n}(x)\right]_{i} \cos \left(\frac{n y}{R}\right),
$$

and the odd fields,

$$
\widetilde{\Phi}_{-}^{(i)}(x)=\sqrt{\frac{1}{\pi R}} \sum_{n=1}^{\infty}\left[\widetilde{\Phi}_{-}^{n}(x)\right]_{i} \sin \left(\frac{n y}{R}\right),
$$

where $\widetilde{\Phi}_{ \pm}^{(i)}, i=1,2$ are the original bulk scalars. Defining further $i=1$ and 2 for positive and negative exponential power one can establish $\widetilde{\Phi}^{(j)}, j=1,2$ with $(+)$ and $(-)$ subscripts respectively and obtain the wavefunction by setting $y \sim 0$ for $\cos \left(\frac{n y}{R}\right)$ as follows,

$$
\begin{aligned}
& \widetilde{\Phi}_{+}^{(1)}(x)=\left.\widetilde{\Phi}_{+}^{(1)}(x, y)\right|_{y \sim 0} \\
& =\left.\frac{1}{\sqrt{\pi R}}\left\{\widetilde{\Phi}_{+,+}^{(0)}(x)+\widetilde{\Phi}_{+,+}^{(n)}(x)\right\} \cos \left(\frac{n y}{R}\right)\right|_{y \sim 0} \\
& =\frac{1}{\sqrt{\pi R}}\left[1+\frac{i f_{2}}{f f_{1}}\left(\begin{array}{ccc}
(0)_{3 \times 3} & \left(\begin{array}{ccc}
0 & 0 & h \\
0 & 0 & h \\
h^{\prime \dagger} & 0
\end{array}\right) \\
\left(\begin{array}{ccc}
0 & 0 & h^{\prime} \\
0 & 0 & 0 \\
h^{\dagger} & 0
\end{array}\right) & \left(\begin{array}{ll}
0 & 0 \\
3 \times 3
\end{array}\right.
\end{array}\right)\right]\left(\begin{array}{l}
0 \\
0 \\
f_{1} \\
0 \\
0 \\
0
\end{array}\right)+
\end{aligned}
$$

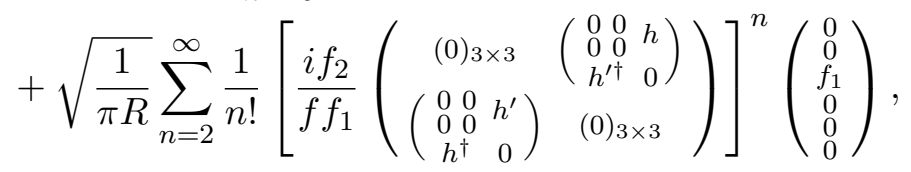

which has $e^{+i \alpha}$-form.

On the other hand, one finds that $\widetilde{\Phi}_{+}^{(2)}$ basically has $e^{-i \alpha}$-form, with the following expansion form as follows,

$$
\begin{aligned}
& \widetilde{\Phi}_{+}^{(2)}(x)=\left.\widetilde{\Phi}_{+}^{(2)}(x, y)\right|_{y \sim 0} \\
& =\left.\frac{1}{\sqrt{\pi R}}\left\{\widetilde{\Phi}_{+,-}^{(0)}(x)+\widetilde{\Phi}_{+,-}^{(n)}(x)\right\} \cos \left(\frac{n y}{R}\right)\right|_{y \sim 0}
\end{aligned}
$$

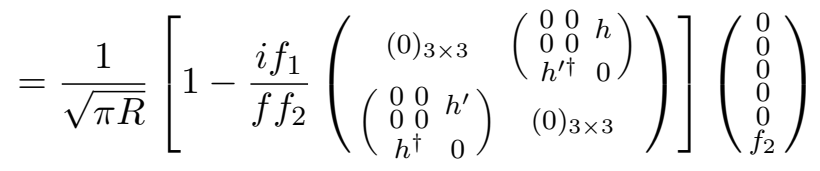

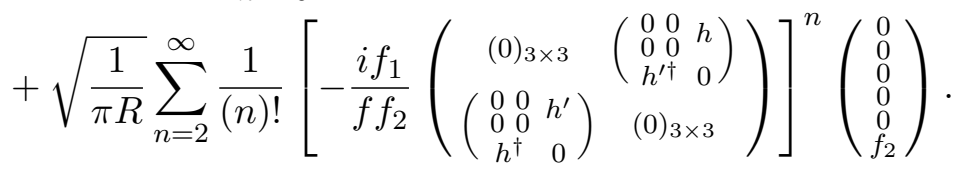


The exponential forms of 5D $(y \sim 0)$ near-brane or 4D SU(6) Little Higgs-like scalars $\widetilde{\Phi}_{+}^{(j)}$ follow directly from Eq. (18) and (19), and are named accordingly as $\mathrm{SU}(6)$ would-be Baby (Little) Higgses as follows,

$$
\widetilde{\Phi}_{+}^{(1)}\left[\widetilde{\Phi}_{+}^{(2)}\right]=\frac{1}{\sqrt{\pi R}} e^{\frac{i f_{2}}{f f_{1}}\left[\frac{-i f_{1}}{f f_{2}}\right]}\left(\begin{array}{ccc}
(0)_{3 \times 3} & \left(\begin{array}{ccc}
0 & 0 & h \\
0 & 0 & \\
h^{\dagger \dagger} & 0
\end{array}\right) \\
\left(\begin{array}{ccc}
0 & 0 & h^{\prime} \\
0 & 0 \\
h^{\dagger} & 0
\end{array}\right) & \left(\begin{array}{ll}
0 & 0 \\
3 \times 3
\end{array}\right.
\end{array}\right)\left(\begin{array}{c}
0 \\
0 \\
f_{1} \\
0 \\
0 \\
0
\end{array}\right)\left[\left(\begin{array}{c}
0 \\
0 \\
0 \\
0 \\
0 \\
f_{2}
\end{array}\right)\right] .
$$

It clearly shows the similarity to the principle of PNBs parametrization in the Simplest Little Higgs $[9,28,29]$. The odd 5D gauge boson in Eq. (17) vanishes in $(y \sim 0)$ near-brane due to $\sin \left(\frac{n y}{R}\right)=0$ for $y \sim 0$.

Next we can also rewrite the wavefunction of SU(6) Little Higgs-like scalar in Eq. (20) utilising the formula from non-linear sigma model and setting accordingly as,

$$
\widetilde{\Phi}_{+}^{(1)}\left[\widetilde{\Phi}_{+}^{(2)}\right]=v\left[v^{\prime}\right] e^{\frac{i f_{2}}{f_{1}}\left[-\frac{i f_{1}}{f_{2}}\right] \theta}
$$

where $v\left[v^{\prime}\right]$ and $\theta$ are already defined in Eq (13) and (15).

Before proceeding further one must realize the following potential problem. Unfortunately dimensional deconstruction without gauge symmetry breaking in 5D $\mathrm{SU}(6) \rightarrow 4 \mathrm{D}$ SU(6) which yields Eq. (18), (19) and (20) happens in 2 (two) equivalent manners i.e. trivial and pseudo non-trivial where in the first no scalar is produced while the second produces scalar. This seemingly contradictory condition is what is exactly needed. The SU(6) would-be Baby (Little) Higgses can not exist too long as required by trivial manner from Eq. (4) righthand where $Z_{2}=U=I_{6}$, so that $\mathrm{KK}$ higher modes are eliminated naturally from Eq. (18), (19) leaving its zero modes as the allowed new scalar by pseudo non-trivial manner in Eq. (4) lefthand [3]. This approach by means of KK higher modes elimination is called weakly-approached.

This can be represented by SU(6) Baby Higgses which are defined by zero mode approximation in the lowest order perturbation. This scalar lives below energy scale $\Lambda_{(6)}^{\mathrm{ZP}} \sim 1000 \mathrm{TeV}, \mathrm{SU}(6)$ Baby Higgses can be written as $\widetilde{\Phi}_{P}^{(i)}, i=1,2(\mathrm{P}$ : perturbative, ZP: zero mode-pertubative) [3].

\subsubsection{SU(6) will-be-SimplestLittleHiggs Scalar}

Let us start with the upper-near-brane Lagrangian which is basically a quartic potential [3] in Eq. (12) where $\lambda_{y}^{(6)}$ is coupling constant of the upper-near-brane while $\widetilde{\Phi}_{+}^{(i)}$ and $\widetilde{\Phi}^{(j)}$ are the SU(6)-origin would be Little (Baby) Higgs bosons expressed as in Eq. (18) and Eq. (19) [3]. The + subscript represents scalar's even parity with the origin from the bulk while no subscript indicating the origin from the fifth component of $5 \mathrm{D}$ gauge boson.

Lagrangian in the lower-near-brane can be obtained from Eq. (12), replacing $\lambda_{y}^{(6)} \rightarrow \lambda_{y \mathrm{NP}}^{(6)}$ and $\widetilde{\Phi}_{+}^{(i)} \rightarrow \widetilde{\Phi}_{+}^{(i)^{\prime}}, \widetilde{\Phi}^{(j)} \rightarrow \widetilde{\Phi}^{(j)^{\prime}}, i=1,2$ and $j=1,2$, as follows,

$$
\mathcal{L}_{y}^{\text {near-brane }}=V_{y \mathrm{NP}}^{(6)}=\lambda_{y \mathrm{NP}}^{(6)} \widetilde{\Phi}_{+}^{(i)^{\prime} \dagger} \widetilde{\Phi}^{(j)^{\prime}} \widetilde{\Phi}^{(j)^{\prime} \dagger} \widetilde{\Phi}_{+}^{(i)^{\prime}},
$$


where $\lambda_{y \mathrm{NP}}^{(6)}$ is coupling constant of lower-near-brane. Now, one utilizes PNB Higgs representation in the exponential form as shown by Eq. (20) and (21) for $\widetilde{\Phi}^{(j)}$, after dropping $(+)$ index. The $\widetilde{\Phi}_{+}^{(i)^{\prime}}, \widetilde{\Phi}^{(j)^{\prime}}$ are the SU(6) will-be-SimplestLittleHiggs bosons which can be expressed as [3],

$$
\widetilde{\Phi}_{+}^{(1)^{\prime}}=\widetilde{\Phi}^{(1)^{\prime}}=\left(\begin{array}{c}
\phi_{0}^{(1)} \\
\phi^{(1)}
\end{array}\right), \quad \widetilde{\Phi}_{+}^{(2)^{\prime}}=\widetilde{\Phi}^{(2)^{\prime}}=\left(\begin{array}{c}
\phi^{(2)} \\
\phi_{0}^{(2)}
\end{array}\right),
$$

and be obtained from, after applying first another approach by means of expanding the (matrix) exponent into an (exponent) matrix which is named accordingly as strongly-approached manner as follows [3,45],

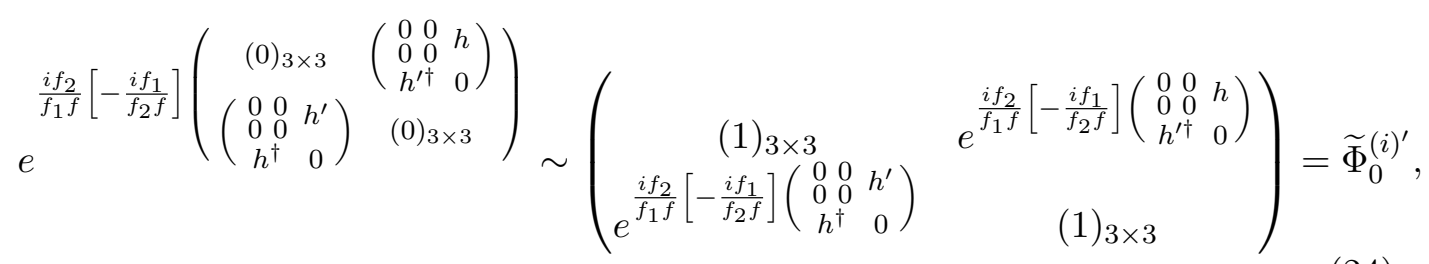

where $i=1,2$ and multiplying it with VEV sextets (Appendix D). Surprisingly the $\mathrm{SU}(6)$ will-be-SimplestLittleHiggs scalars experience a splitting into weakly-coupled zero mode VEV triplet $\phi_{0}^{(1)}\left[\phi_{0}^{(2)}\right]$ and a strongly-coupled Little Higgs-like triplet $\phi^{(1)}\left[\phi^{(2)}\right]$ similar to triplet-doublet splitting of 5D SU(5) Higgs quintet [48].

One finds basically SU(3) VEV triplets acting for the weakly-coupled zero mode triplets and SU(3) SimplestLittleHiggs-like scalar for the strongly-coupled full-mode triplets with the following expressions,

$$
\phi_{0}^{(1)}\left[\phi_{0}^{(2)}\right]=\frac{1}{\sqrt{\pi R}}\left(\begin{array}{c}
0 \\
0 \\
f_{1}
\end{array}\right)\left[\left(\begin{array}{c}
0 \\
0 \\
f_{2}
\end{array}\right)\right]=\left(\begin{array}{c}
0 \\
0 \\
f_{1}^{\prime}
\end{array}\right)\left[\left(\begin{array}{c}
0 \\
0 \\
f_{2}^{\prime}
\end{array}\right)\right],
$$

and below.

$$
\phi^{(1)}=e^{\frac{i f_{2}^{\prime}}{f_{1}^{\prime} f^{\prime}}\left(\begin{array}{ccc}
0 & 0 & H^{\prime} \\
0 & 0 & H^{\dagger} \\
H^{\dagger} & 0
\end{array}\right)}\left(\begin{array}{c}
0 \\
0 \\
f_{1}^{\prime}
\end{array}\right), \quad \phi^{(2)}=e^{-\frac{i f_{1}^{\prime}}{f_{2}^{\prime} f^{\prime}}\left(\begin{array}{ccc}
0 & 0 & H \\
0 & 0 & 0 \\
H^{\prime \dagger} & 0
\end{array}\right)}\left(\begin{array}{c}
0 \\
0 \\
f_{2}^{\prime}
\end{array}\right),
$$

where $f_{i}^{\prime}=\frac{1}{\sqrt{\pi R}} f_{i}, f^{\prime 2}=f_{1}^{\prime 2}+f_{2}^{\prime 2}, H\left(H^{\prime}\right)=\frac{1}{\sqrt{\pi R}} h\left(h^{\prime}\right)$, and $\theta \rightarrow \theta^{\prime}$ as,

$$
\theta^{\prime}=\frac{1}{f^{\prime}}\left(\begin{array}{cc}
(0)_{3 \times 3} & \left(\begin{array}{ccc}
0 & 0 & H \\
0 & 0 & H \\
H^{\prime \dagger} & 0
\end{array}\right) \\
\left(\begin{array}{ccc}
0 & 0 & H^{\prime} \\
0 & 0 & (0)_{3 \times 3} \\
H^{\dagger} & 0
\end{array}\right) & \left(\begin{array}{ll}
0 \\
3 \times 3
\end{array}\right.
\end{array}\right)=\theta^{\prime \dagger}
$$

With these new variables Eq. (21) can be re-written as (dropping index + ),

$$
\widetilde{\Phi}^{(1)}\left[\widetilde{\Phi}^{(2)}\right]=v\left[v^{\prime}\right] e^{\frac{i f_{2}^{\prime}}{f_{1}^{\prime}}\left[-\frac{i f_{1}^{\prime}}{f_{2}^{\prime}}\right]},
$$


which suits the required condition of triplet-triplet splitting better, as indicated by Eq. (24) after VEV multiplication. This means that SU(6) will-be-SimplestLittle Higgs scalar is only a transitional state. It is another strongly-coupled Little Higgslike suffering from large possibility to undergo triplet-triplet splitting after some moments.

Therefore, under the requirements of trivial and pseudo non-trivial manners, $\widetilde{\Phi}^{(1)^{\prime}}$ and $\widetilde{\Phi}^{(2)^{\prime}}$ can not last too long and have to be converted into a newly-formed scalars which, in this strongly-approached manner, are achieved through the triplettriplet splitting as shown above by Eq. (25) and (26). One defines accordingly the following parameters for SU(3) SimplestLittleHiggs-like scalar as,

$$
\begin{aligned}
& \theta_{1}^{\prime}=\frac{1}{f^{\prime}}\left(\begin{array}{ccc}
0 & 0 & H^{\prime} \\
0 & 0 & H^{\dagger} \\
H^{\dagger} & 0
\end{array}\right)=\frac{1}{f^{\prime}}(H)_{\mathrm{pnb}}^{\dagger}, \\
& \theta_{2}^{\prime}=\frac{1}{f^{\prime}}\left(\begin{array}{ccc}
0 & 0 & H \\
0 & 0 & H \\
H^{\prime \dagger} & 0
\end{array}\right)=\frac{1}{f^{\prime}}(H)_{\mathrm{pnb}}=\theta_{1}^{\prime \dagger},
\end{aligned}
$$

with which Eq. (26) and Eq. (27) can be written compactly as,

$$
\begin{aligned}
\phi^{(1)}\left[\phi^{(2)}\right] & =\phi_{0}^{(1)}\left[\phi_{0}^{(2)}\right] e^{\frac{i i f_{1}^{\prime}}{f_{1}^{\prime} f^{\prime}}\left[\frac{-i f_{1}^{\prime}}{f_{2}^{\prime} f^{\prime}}\right]} \theta_{1}^{\prime}\left[\theta_{2}^{\prime}\right] \\
\theta^{\prime} & =\left(\begin{array}{cc}
(0)_{3 \times 3} & \theta_{2}^{\prime} \\
\theta_{1}^{\prime} & (0)_{3 \times 3}^{\prime}
\end{array}\right) .
\end{aligned}
$$

The (asymptotic) shift symmetry can be seen clearly from the application of global gauge transformation at $\widetilde{\Phi}^{(i)}, i=1,2$ by means of $e^{i \alpha Q}$ where $Q$ is the broken generators. The (asymptotic) shift symmetry and its breaking terms can be seen from expressions, expanding $e^{i \alpha Q} \widetilde{\Phi}^{(i)}, i=1,2$ with $\widetilde{\Phi}^{(i)}$ as given by Eq. (28), as below

$$
\begin{aligned}
\widetilde{\Phi}^{(1)}: \frac{f_{2}^{\prime}}{f_{1}^{\prime}} \theta^{\prime} \rightarrow & \frac{f_{2}^{\prime}}{f_{1}^{\prime}} \theta^{\prime}+Q \alpha+i\left\{\frac{f_{2}^{\prime}}{f_{1}^{\prime}} \theta^{\prime} Q \alpha+([1]+i Q \alpha) \frac{f_{2}^{\prime 2}}{2 f_{1}^{\prime 2}} \theta^{\prime 2}\right\} \\
& +([1]+i Q \alpha) \mathcal{O}\left(\theta^{\prime 3}\right), \\
\widetilde{\Phi}^{(2)}:-\frac{f_{1}^{\prime}}{f_{2}^{\prime}} \theta^{\prime} \rightarrow & -\frac{f_{1}^{\prime}}{f_{2}^{\prime}} \theta^{\prime}+Q \alpha-i\left\{\frac{f_{1}^{\prime}}{f_{2}^{\prime}} \theta^{\prime} Q \alpha-([1]+i Q \alpha) \frac{f_{1}^{\prime 2}}{2 f_{2}^{\prime 2}} \theta^{\prime 2}\right\} \\
& +([1]+i Q \alpha) \mathcal{O}\left(\theta^{\prime 3}\right) .
\end{aligned}
$$

\section{On the $\mathrm{SU}(6)$ 5D-twist Nambu-Goldstone boson and its role in the formation of Scherk-Schwarz scalar}

\subsection{SU(6) 5D-twist NGB and Generalized Coleman- Weinberg po- tential}

Considering Eq. (8) the orbifold $S^{1} / Z_{2}$ provides equation $y / R=\theta$ where $\theta$ is the sectoral angle at the $S^{1}$ center so that one rewrites infinitesimally the above equation 
as,

$$
\delta \alpha=\omega \delta \theta .
$$

The $\delta \alpha-\delta \theta$ correspondence in Eq. (35) dictates the existing correspondence of 5D $(y \sim 0)$ broken fifth component of 5D gauge boson $A_{y}^{\hat{a}}$ and the 5D-twist Nambu-Goldstone boson while the first also relates directly to Higgs boson under the principle of gauge-Higgs unification. This means that the correspondence extends between 4D PNB Higgs and 5D $(y \sim 0)$ Nambu-Goldstone boson (NGB) due to global and local properties. Consequently can one generalize and interprete $\widetilde{\Phi}^{(j)}, j=1,2$ in Eq. (12) to include NGB, $\xi^{(j)}$, which can be put in a diagonal matrix representation, following consideration for PNBs residing in the off-diagonal sub-matrices as shown by Eq. (15).

Therefore one can utilize, without loss of generality, $\mathrm{SU}(6)$ generators $\lambda_{8}, \lambda_{34}$, and $\lambda_{35}$ for this purpose and defining NGBs as follows,

$$
\xi^{(1)}=\xi\left(n_{8} \lambda_{8}+n_{35} \lambda_{35}\right), \quad \xi^{(2)}=\xi\left(n_{34} \lambda_{34}+n_{35} \lambda_{35}\right),
$$

where $n_{8}, n_{34}$ and $n_{35}$ are normalization constants and the generators are provided below [1],

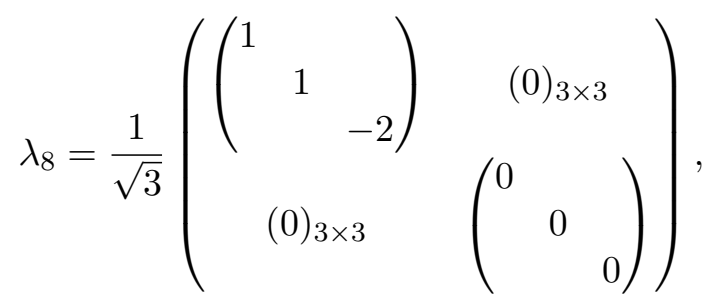

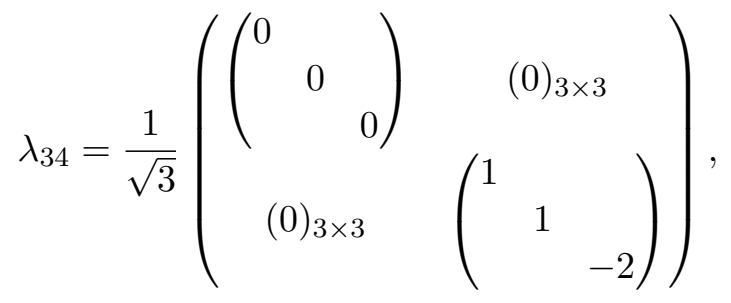

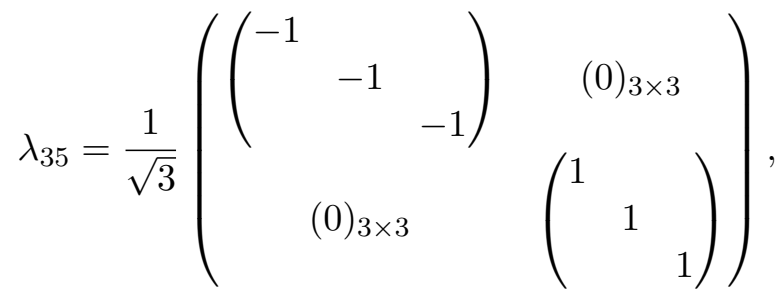

so that one obtains finally,

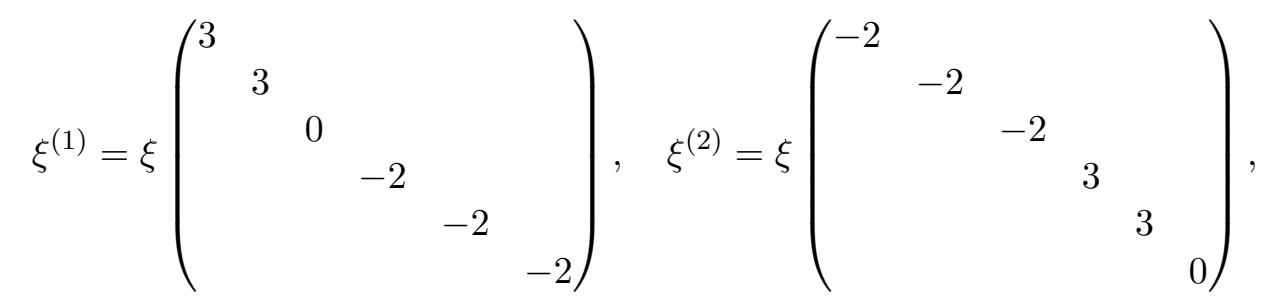


where $\xi$ is NGB, making use definitions as follows: $\xi^{\prime}=3 \xi / \sqrt{\pi R}, \xi_{0}=-2 \xi / \sqrt{\pi R}$ and $\left(\xi^{\prime}\right)_{\mathrm{ng}}=\left(\begin{array}{ccc}\xi^{\prime} & & \\ & \xi^{\prime} & \\ & & 0\end{array}\right), \xi_{0}^{\prime}=\xi_{0}\left(\begin{array}{lll}1 & & \\ & & 1\end{array}\right), \mathrm{SU}(6)$ 5D-twist NGBs in short form as,

$$
\xi^{\prime(1)}=\left(\begin{array}{cc}
\left(\left(\xi^{\prime}\right)_{\mathrm{ng}}\right)_{3 \times 3} & (0)_{3 \times 3} \\
(0)_{3 \times 3} & \left(\left(\xi^{\prime}\right)_{0}\right)_{3 \times 3}
\end{array}\right), \quad \xi^{\prime(2)}=\left(\begin{array}{cc}
\left(\left(\xi^{\prime}\right)_{0}\right)_{3 \times 3} & (0)_{3 \times 3} \\
(0)_{3 \times 3} & \left(\left(\xi^{\prime}\right)_{\mathrm{ng}}\right)_{3 \times 3}
\end{array}\right) .
$$

Recalling Eq. (12) with $i \neq j$ Coleman-Weinberg potential is generalized by replacing $\widetilde{\Phi}^{(j)}, j=1,2$ with $\Phi_{\mathrm{NG}}^{(j)}, j=1,2$ which is quite justified considering $\Phi_{\mathrm{NG}}^{(j)}, j=1,2$ is the third component of a massive $4 \mathrm{D}$ gauge boson in the electroweak theory while $\widetilde{\Phi}^{(i)}, i=1,2$ PNB Higgs.

Substitution of the originally-set fifth component of $5 \mathrm{D}$ gauge boson with its third component is naturally logical and definitely acceptable [26] which gives a generalized Coleman-Weinberg potential for SU(6) would-be Baby (Little) Higgs [3] as below,

$$
V_{y g}^{(6)}=\lambda_{y}^{(6)}\left(\widetilde{\Phi}^{(i) \dagger} \Phi_{\mathrm{NG}}^{(j)}\right)\left(\Phi_{\mathrm{NG}}^{(j) \dagger} \widetilde{\Phi}^{(i)}\right), \quad i, j=1,2,
$$

and for SU(6) will-be-SimplestLittleHiggs scalar [3] as,

$$
V_{\mathrm{NP} g}^{(6)}=\lambda_{y \mathrm{NP}}^{(6)}\left(\widetilde{\Phi}^{(i)^{\prime} \dagger} \Phi_{\mathrm{NG}}^{(j)}\right)\left(\Phi_{\mathrm{NG}}^{(j) \dagger} \widetilde{\Phi}^{(i)^{\prime}}\right), \quad i, j=1,2,
$$

where $\Phi_{\mathrm{NG}}^{(j)}$ is written as,

$$
\Phi_{\mathrm{NG}}^{(j)}=v_{\mathrm{NG}}^{(j)} e^{ \pm \frac{i f_{i}^{\prime} f_{j}^{\prime} \xi^{\prime(j)}}{f^{(j)}}}, \quad i \neq j=1,2,
$$

where $(+)$ for $i=1,(-)$ for $i=2$ and $v_{\mathrm{NG}}^{(1)}=v$ and $v_{\mathrm{NG}}^{(2)}=v^{\prime}$ in Eq. (13) or $v_{\mathrm{NG}}^{(1)}=\left(\begin{array}{llllll}0 & 0 & f_{1}^{\prime} & 0 & 0 & 0\end{array}\right)^{T}$ and $v_{\mathrm{NG}}^{(2)}=\left(\begin{array}{llllll}0 & 0 & 0 & 0 & 0 & f_{2}^{\prime}\end{array}\right)^{T}$. Following Eq. (24) and expanding the (matrix) exponent in Eq. (42) into an (exponent) matrix in the strongly-approached manner after substituting with Eq. (39) one gets the result as,

$$
\begin{aligned}
e^{ \pm \frac{i f_{i}^{\prime}}{f_{j}^{\prime} f^{\prime}} \xi^{\prime(j)}} & =e^{-\frac{i f_{2}^{\prime}}{f_{1}^{\prime} f^{\prime}}\left[\frac{i f_{1}^{\prime}}{f_{2}^{\prime} f^{\prime}}\right]\left(\begin{array}{cc}
\xi_{\text {ng }}^{\prime} & 0 \\
0 & \xi_{0}^{\prime}
\end{array}\right)\left[\left(\begin{array}{cc}
\xi_{0}^{\prime} & 0 \\
0 & \xi_{\text {ng }}^{\prime}
\end{array}\right)\right]} \\
& \sim\left(\begin{array}{cc}
e^{-\frac{i f_{2}^{\prime}}{f_{1}^{\prime} f^{\prime}} \xi_{\text {ng }}^{\prime}} & 1 \\
1 & e^{-\frac{i f_{2}^{\prime}}{f_{1}^{\prime} f^{\prime}} \xi_{0}^{\prime}}
\end{array}\right)\left[\left(\begin{array}{cc}
e^{i f_{1}^{\prime} f^{\prime}} \xi_{0}^{\prime} & 1 \\
1 & e^{\frac{i f_{2}^{\prime}}{f_{2}^{\prime} f^{\prime}} \xi_{\text {ng }}^{\prime}}
\end{array}\right)\right],
\end{aligned}
$$

which brings directly to, with the aid of Eq. (13), the following sextets,

$$
\Phi_{\mathrm{NG}}^{(1)^{\prime}}=\left(\begin{array}{c}
e^{-\frac{i f_{2}^{\prime}}{f_{1}^{\prime} f^{\prime}} \xi_{\mathrm{ng}}^{\prime}}\left(\begin{array}{c}
0 \\
0 \\
f_{1}^{\prime}
\end{array}\right) \\
\left(\begin{array}{c}
0 \\
0 \\
f_{1}^{\prime}
\end{array}\right)
\end{array}\right), \quad \Phi_{\mathrm{NG}}^{(2)^{\prime}}=\left(\begin{array}{c}
\left(\begin{array}{c}
0 \\
0 \\
f_{2}^{\prime}
\end{array}\right) \\
i \frac{i f_{1}^{\prime}}{f_{2}^{\prime} \xi_{\mathrm{ng}}^{\prime}}\left(\begin{array}{c}
0 \\
0 \\
f_{2}^{\prime}
\end{array}\right)
\end{array}\right),
$$

where the prime is to indicate the strong manner application. From Eq. (44) one can define $\mathrm{SU}(3) \operatorname{NGBs} \phi_{\mathrm{NG}}^{(j)}, j=1,2$ as follows,

$$
\phi_{\mathrm{NG}}^{(1)}\left[\phi_{\mathrm{NG}}^{(2)}\right]=e^{-\frac{i f_{2}^{\prime}}{f_{1}^{\prime} f^{\prime}}\left[\frac{i f_{1}^{\prime}}{f_{2}^{\prime} f^{\prime}}\right] \xi_{\mathrm{ng}}^{\prime}}\left(\begin{array}{c}
0 \\
0 \\
f_{1}^{\prime}
\end{array}\right)\left[\left(\begin{array}{c}
0 \\
0 \\
f_{2}^{\prime}
\end{array}\right)\right], \quad \xi_{\mathrm{ng}}^{\prime}=\left(\begin{array}{ccc}
\xi^{\prime} & & \\
& \xi^{\prime} & \\
& & 0
\end{array}\right) .
$$


If factor $1 / \sqrt{2}$ is allowed to be absorbed by $\left(\xi_{\text {ng }}^{\prime}\right)_{j j}=\left(\frac{1}{\sqrt{2}} \xi^{\prime}\right), j=1,2$, then Eq. (45) resembles completely NGBs produced in $\mathrm{SU}(3) \times \mathrm{SU}(3)$ symmetry as discussed in next section.

As a matter of fact, one can rewrite Eq. (44) into a nice compact form similar to Eq. (23) as below,

$$
\Phi_{\mathrm{NG}}^{(1)^{\prime}}=\left(\begin{array}{c}
\phi_{\mathrm{NG}}^{(1)} \\
\phi_{0}^{(1)}
\end{array}\right), \quad \Phi_{\mathrm{NG}}^{(2)^{\prime}}=\left(\begin{array}{c}
\phi_{0}^{(2)} \\
\phi_{\mathrm{NG}}^{(2)}
\end{array}\right)
$$

In comparison with Eq. (23) one finds that doublets in Eq. (46) above are the inversion of doublets in Eq. (23) after replacing $\phi_{\mathrm{NG}}^{(j)}$ with $\phi^{(i)}, i, j=1,2$, indicating 2 (two) different 2-VEV vacuum states where one belongs to PNB Higgs field and another one to NGB field or its derivates with doublet of the second is the inversion of the first's.

Summing up Eq. (23) and (46), the following result is obtained,

$$
\widetilde{\Phi}^{(1)^{\prime}}+\Phi_{\mathrm{NG}}^{(1)}=\left(\begin{array}{c}
\phi_{0}^{(1)}+\phi_{\mathrm{NG}}^{(1)} \\
\phi_{0}^{(1)}+\phi^{(1)}
\end{array}\right), \quad \widetilde{\Phi}^{(2)^{\prime}}+\Phi_{\mathrm{NG}}^{(2)}=\left(\begin{array}{c}
\phi_{0}^{(2)}+\phi^{(2)} \\
\phi_{0}^{(2)}+\phi_{\mathrm{NG}}^{(2)}
\end{array}\right),
$$

where each VEV $\phi_{0}^{(i)}, i=1,2$ is shifted by both Higgs shift and NGB shift indicating the existence of double vacua with 2 -VEV system and inverted vacuum states $[36,45]$.

\subsection{Predicted emergence of Scherk-Schwarz scalar}

With the aid of Eq. (28) and (42) the generalized Coleman-Weinberg potential in Eq. (12) is computed, taking $i=j=1,2$ for this purpose, so that one starts with,

$$
V_{y g}^{(6)}=V_{1}^{(6)}+V_{2}^{(6)}=\lambda_{y}^{(6)}\left(\widetilde{\Phi}^{(1) \dagger} \Phi_{\mathrm{NG}}^{(1)}\right)\left(\Phi_{\mathrm{NG}}^{(1) \dagger} \widetilde{\Phi}^{(1)}\right)+\lambda_{y}^{(6)}\left(\widetilde{\Phi}^{(2) \dagger} \Phi_{\mathrm{NG}}^{(2)}\right)\left(\Phi_{\mathrm{NG}}^{(2) \dagger} \widetilde{\Phi}^{(2)}\right),
$$

where $V_{1}^{(6)}$ and $V_{2}^{(6)}$ are found as below,

$$
V_{1}^{(6)}\left[V_{2}^{(6)}\right]=\lambda_{y}^{(6)}\left(v^{T} v\right)^{2}\left[\left(v^{\prime T} v^{\prime}\right)^{2}\right] e^{\frac{i f_{2}^{\prime}}{f_{1}^{\prime}}\left[-\frac{i f_{1}^{\prime}}{f_{2}^{\prime}}\right]\left\{\left(\theta^{\prime}-\theta^{\prime \dagger}\right)+\frac{1}{f^{\prime}}\left(\xi^{\prime(1) \dagger}\left[\xi^{(2)}\right]-\xi^{\prime(1)}\left[\xi^{(2) \dagger}\right]\right)\right\}},
$$

with $\theta^{\prime}=\theta^{\prime \dagger}$ and $\xi^{\prime(i)}=\xi^{\prime(i) \dagger}, i=1,2$ for $\mathrm{SU}(6)$. Special note must be taken here that scalars $\widetilde{\Phi}^{(i) \dagger}, \widetilde{\Phi}^{(i)}, i=1,2$ in Eq. (48) are SU(6) would-be Baby (Little) Higgses which must decouple from the system. No wonder, the potentials in Eq. (49) are zero (or constant).

In order to apply the strongly-coupled condition and facilitate triplet-triplet splitting which is induced by the emerging of SU(6) will-be-SimplestLittleHiggs scalars [48] then potentials in Eq. (48) and (49) become non-zero which will be discussed in the next subsection. Meanwhile, it is adequate for the moment to consider the inequality between Eq. (12) and (22) is due to different physical conditions inherited from the changing from sextet-based to triplet-based as dictated by the will-be triplet-triplet splitting [48] which provides finally the zero and non-zero potentials. 
Consequently Eq. (49) must be adjustable and can be reformed to accommodate the non-zero potential, at least, at the switching from Eq. (12) into (49). Let's rewrite Eq. (22) and (49) in the following compact form, setting $\lambda_{y}^{(6)} \sim \lambda_{y \mathrm{NP}}^{(6)}$, as

$$
V_{1}^{(6)}\left[V_{2}^{(6)}\right]=\lambda_{y \mathrm{NP}}^{(6)} f_{1}^{\prime 4}\left[f_{2}^{\prime 4}\right] e^{\frac{i f_{2}^{\prime}}{f_{1}^{\prime}}\left[\frac{i f_{1}^{\prime}}{f_{2}^{\prime}}\right]\left\{\left(\theta^{\prime} \pm \frac{1}{f^{\prime}} \xi^{\prime(1)}\left[\xi^{\prime(2)}\right]\right)-\left(\theta^{\prime \dagger} \pm \frac{1}{f^{\prime}} \xi^{(1) \dagger}\left[\xi^{(2) \dagger}\right]\right)\right\}},
$$

where \pm means $-\xi^{\prime(1)}\left(\xi^{\prime(1) \dagger}\right)$ and $+\xi^{\prime(2)}\left(\xi^{\prime(2) \dagger}\right)$ which are important property showing up in triplet-based due to $\theta^{\prime} \neq \theta^{\prime \dagger}$ as shown below

$$
\theta_{1}^{\prime}=\frac{1}{f^{\prime}}\left(\begin{array}{ccc}
0 & 0 & H^{\prime} \\
0 & 0 & H^{\dagger} \\
0
\end{array}\right), \quad \theta_{2}^{\prime}=\theta_{1}^{\prime \dagger}=\frac{1}{f^{\prime}}\left(\begin{array}{ccc}
0 & 0 & H \\
0 & 0 & H \\
H^{\prime \dagger} & 0
\end{array}\right),
$$

while $\xi^{\prime(1)}\left(\xi^{\prime(2)}\right)$ becomes $\xi_{n g}^{\prime}=\xi_{n g}^{\prime \dagger}$. This is the reason to keep $\theta^{\prime \dagger}$, or the will-be $\theta_{i}^{\prime \dagger}, i=1,2$, in Eq. (49) in order to have a more general form. The non-zero potential in Eq. (50) indicates the taking place of triplet-triplet splitting and emerging of a new scalar triplet which is defined accordingly as,

$$
\left(H_{0}^{\prime \prime}\right)=\left(\theta_{1}^{\prime} \pm \xi_{n g}^{\prime}\right)-\left(\theta_{1}^{\prime \dagger} \pm \xi_{n g}^{\prime}\right),
$$

where $\left(H_{0}^{\prime \prime}\right)$ is the will-be Scherk-Schwarz scalar in $3 \times 3$ matrix. Eq. (52) produces a triplet of gauge-like scalars which means and further defines that Scherk-Schwarz scalar triplet is formed from the unification of 1 (one) PNB Higgs triplet and 2 (two) NGB triplets.

Comparing SU(3) to SU(2) Eq. (52) facilitates for establishing a one-to-one correspondence as follows,

$$
\theta_{1}^{\prime}\left[\theta_{1}^{\prime \dagger}\right] \longleftrightarrow H^{\prime}[H], \quad \xi_{n g}^{\prime} \longleftrightarrow \xi^{\prime}, \quad\left(H_{0}^{\prime \prime}\right) \longleftrightarrow H_{0}^{\prime \prime},
$$

where $H^{\prime}[H], \xi^{\prime}, H_{0}^{\prime \prime}$ are doublets so that one can predict the existence of the following generalized Scherk-Schwarz scalars, based on Eq. (52), as

$$
H_{0}^{\prime \prime}=\left(H^{\prime} \pm \xi^{\prime}\right) \pm\left(H \pm \xi^{\prime}\right)
$$

which shows the unification of one new-Higgs field $H^{\prime \prime}=\left(H^{\prime}-H\right)$ with the 2 (two) NGBs where the second has vanished into $H^{\prime \prime}$ to result $H_{0}^{\prime \prime}$ or $H^{\prime \prime}=\left(H^{\prime}+H\right)$ with 2 (two) unvanished NGBs.

\section{On the double-vacua shift symmetry and Scherk- Schwarz twist breaking}

\subsection{The double-vacua shift and twist factor in the near-brane}

From the non-linear sigma model with Kibble parameterization both Higgs $H\left[H^{\prime}\right]$ and NGB $\xi^{\prime}$ can be expressed as the shifts of VEV, $f$, which can be written as $f \rightarrow f+\epsilon H\left[H^{\prime}\right]$ and $0 \rightarrow 0+\epsilon \xi^{\prime}$ with $\epsilon$ any very small positive number. Summing up both shifts one obtains immediately the following

$$
f \rightarrow f+\epsilon\left\{H\left[H^{\prime}\right]+\xi^{\prime}\right\},
$$


which shows the existence of multi vacua due to NGB shift symmetry. In this case, Eq. (55) is called the double-vacua shift symmetry. Generalizing Eq. (55) to SU(6) and $\mathrm{SU}(3)$, one can rewrite Eq. (55), replacing $f \rightarrow v_{k}\left[v_{k}^{\prime}\right]$ with $k=6,3$ for $\mathrm{SU}(6)$ and SU(3) successively, $H\left[H^{\prime}\right] \rightarrow\left[(H)_{6 \times 6}\right]$ and $H\left[H^{\prime}\right] \rightarrow(H)_{3 \times 3}\left[\left(H^{\prime}\right)_{3 \times 3}\right]$ for $\mathrm{SU}(6)$ and $\mathrm{SU}(3)$ while $\xi^{\prime} \rightarrow \xi^{(i)^{\prime}}$ for $\mathrm{SU}(6)$ and $\xi^{\prime} \rightarrow \xi_{n g}^{\prime}$ for $\mathrm{SU}(3)$, as follows,

$$
\begin{aligned}
& v_{6}\left[v_{6}^{\prime}\right] \rightarrow v_{6}\left[v_{6}^{\prime}\right]+\epsilon\left\{(H)_{6 \times 6}+\xi^{(i)^{\prime}}\right\}, \\
& v_{3}\left[v_{3}^{\prime}\right] \rightarrow v_{3}\left[v_{3}^{\prime}\right]+\epsilon\left\{(H)_{3 \times 3}\left[\left(H^{\prime}\right)_{3 \times 3}\right]+\xi_{n g}^{\prime}\right\} .
\end{aligned}
$$

Defining $\theta\left(=\theta^{\prime}\right)=\frac{1}{f}(h)_{6 \times 6}=\frac{1}{f^{\prime}}(H)_{6 \times 6}, \theta_{1}^{\prime}=\frac{1}{f^{\prime}}\left(H^{\prime}\right)_{3 \times 3}, \theta_{2}^{\prime}=\theta_{1}^{\prime \dagger}=\frac{1}{f^{\prime}}(H)_{3 \times 3}$ and setting $\epsilon=\frac{1}{f^{\prime}}$, Eq. (56) finally becomes,

$$
\begin{aligned}
& v_{6}\left[v_{6}^{\prime}\right] \rightarrow v_{6}\left[v_{6}^{\prime}\right]+\left\{\theta^{\prime}+\frac{1}{f^{\prime}} \xi^{(i)^{\prime}}\right\}, \\
& v_{3}\left[v_{3}^{\prime}\right] \rightarrow v_{3}\left[v_{3}^{\prime}\right]+\left\{\theta_{2}^{\prime}\left[\theta_{1}^{\prime}\right]+\frac{1}{f^{\prime}} \xi_{n g}^{\prime}\right\} .
\end{aligned}
$$

In this model, the Little Higgs-like scalars of SU(6) and $\mathrm{SU}(3)$, based on nonlinear sigma model, have parametrization in the phase factors as shown in Eq. (28) and Eq. (31) where parameters $\theta^{\prime}$ and $\theta_{i}^{\prime}, i=1,2$ are replaced by the shifts in Eq. (57) so that double-vacua wavefunctions are obtained. Under expansion the exponential double-vacua wavefunctions modify itselves into, expanding to the lowest order, the following,

$$
\begin{aligned}
& v_{6}\left[v_{6}^{\prime}\right] \rightarrow v_{6}\left[v_{6}^{\prime}\right]\left[1+\left\{\theta^{\prime}+\frac{1}{f^{\prime}} \xi^{(i)^{\prime}}\right\}\right], \\
& v_{3}\left[v_{3}^{\prime}\right] \rightarrow v_{3}\left[v_{3}^{\prime}\right]\left[1+\left\{\theta_{2}^{\prime}\left[\theta_{1}^{\prime}\right]+\frac{1}{f^{\prime}} \xi_{n g}^{\prime}\right\}\right],
\end{aligned}
$$

omitting for the moment the factors $\frac{i f_{2}^{\prime}}{f_{1}^{\prime}}$ and $\frac{-i f_{1}^{\prime}}{f_{2}^{\prime}}$. Defining these factors $F_{6}^{\prime}=$ $1+\left\{\theta^{\prime}+\frac{1}{f^{\prime}} \xi^{(i)^{\prime}}\right\}$ and $F_{3}^{\prime}=1+\left\{\theta_{2}^{\prime}\left[\theta_{1}^{\prime}\right]+\frac{1}{f^{\prime}} \xi_{n g}^{\prime}\right\}$ one can rewrite Eq. (28) and Eq. (31) for double-vacua wavefunctions in the lowest order mode as follows,

$$
\widetilde{\Phi}^{(1)}\left[\widetilde{\Phi}^{(2)}\right] \sim v\left[v^{\prime}\right] F_{6}^{\prime}, \quad \phi^{(1)}\left[\phi^{(2)}\right] \sim \phi_{0}^{(1)}\left[\phi_{0}^{(2)}\right] F_{3}^{\prime} .
$$

where the following identities $v_{6}\left[v_{6}^{\prime}\right]=v\left[v^{\prime}\right]$ and $v_{3}\left[v_{3}^{\prime}\right]=\phi_{0}^{(1)}\left[\phi_{0}^{(2)}\right]$ have been applied.

On the contrary an exact form can also be obtained easily, defining $A\left[A^{\dagger}\right]=$ $\theta^{\prime}\left[\theta^{\prime \dagger}\right], B\left[B^{\dagger}\right]=\frac{1}{f^{\prime}} \xi^{\prime(j)}\left[\xi^{\prime(j) \dagger}\right], j=1,2$ for $\mathrm{SU}(6)$ and $A\left[A^{\dagger}\right]=\theta_{2}^{\prime}\left[\theta_{1}^{\prime}\right], B\left[B^{\dagger}\right]=\frac{1}{f^{\prime}} \xi_{n g}^{\prime}\left[\xi_{n g}^{\prime \dagger}\right]$, for SU(3), Eq. (28) and Eq. (31) can be expressed separately, still omitting the factors $\frac{i f_{2}^{\prime}}{f_{1}^{\prime}}$ and $\frac{-i f_{1}^{\prime}}{f_{2}^{\prime}}$, as

$$
\widetilde{\Phi}^{(1)}\left[\widetilde{\Phi}^{(2)}\right]=v\left[v^{\prime}\right] e^{A}, \quad \phi^{(1)}\left[\phi^{(2)}\right]=\phi_{0}^{(1)}\left[\phi_{0}^{(2)}\right] e^{A^{\dagger}[A]},
$$

while for Eq. (42) and Eq. (45), still omitting factors $\frac{-i f_{2}^{\prime}}{f_{1}^{\prime}}$ and $\frac{i f_{1}^{\prime}}{f_{2}^{\prime}}$, as

$$
\Phi_{\mathrm{NG}}^{(j)}=v\left[v^{\prime}\right] e^{B}, \quad \phi_{\mathrm{NG}}^{(j)}=\phi_{0}^{(j)} e^{B}, j=1,2,
$$


taking into notice $A=A^{\dagger}, B=B^{\dagger}$ for $\mathrm{SU}(6)$ while $A \neq A^{\dagger}, B=B^{\dagger}$ for $\mathrm{SU}(3)$ where $A\left[A^{\dagger}\right]$ and $B\left[B^{\dagger}\right]$ are operators or operator-like generators.

In view of the products $\left(\widetilde{\Phi}^{(i) \dagger} \Phi_{\mathrm{NG}}^{(j)}\right)$ and $\left(\Phi_{\mathrm{NG}}^{(j) \dagger} \widetilde{\Phi}^{(i)}\right), i, j=1,2$ in Eq. (40) each product is rewritten, making use Eq. (60) and Eq. (61) where generators in the exponential functions are treated accordingly as exponential operators following BakerCampbell-Hausdorff (BCH) formula [31] as below

$$
\begin{aligned}
\widetilde{\Phi}^{(i) \dagger} \Phi_{\mathrm{NG}}^{(j)} & =v^{T}\left[v^{\prime T}\right] v\left[v^{\prime}\right]\left[v^{\prime}[v]\right] e^{A^{\dagger}} e^{B}\left[e^{B^{\dagger}}\right] \\
\Phi_{\mathrm{NG}}^{(j) \dagger} \widetilde{\Phi}^{(i)} & =v^{T}\left[v^{T}\right]\left[v^{\prime T}\left[v^{T}\right]\right] v\left[v^{\prime}\right] e^{B}\left[e^{B^{\dagger}}\right] e^{A}, \quad i, j=1,2,
\end{aligned}
$$

with $e^{A^{\dagger}} e^{B} \sim e^{A^{\dagger}+B+\frac{1}{2}\left[A^{\dagger}, B\right]}$ or $e^{A} e^{B} \sim e^{A+B+\frac{1}{2}[A, B]}[31,45]$ which can finally be substituted into Eq. (40) to give the $\mathrm{SU}(6)$ LittleHiggs-like potential as,

$$
V_{y g}^{(6)}=2 \lambda_{y}^{(6)}\left\{v^{T}\left[v^{\prime T}\right] v\left[v^{\prime}\right]\right\}^{2}\left[\left\{v^{T}\left[v^{\prime T}\right] v^{\prime}[v]\right\}^{2}\right] F_{6},
$$

following Appendix I where $V_{y g}^{(6)}=V_{c w}$ and $F_{6}=\frac{1}{2} F$.

For SU(3), Little Higgs-like potential can easily be obtained from Eq. (63) by replacing $v \rightarrow \phi_{0}^{(1)}, v^{\prime} \rightarrow \phi_{0}^{(2)}$ and $F_{6} \rightarrow F_{3}$ with further calculation has been given in Appendix I.

Recalling Appendix I, and substituting as above $A\left[A^{\dagger}\right]=\theta^{\prime}\left[\theta^{\prime \dagger}\right], \quad B\left[B^{\dagger}\right]=$ $\frac{1}{f^{\prime}} \xi^{(i)^{\prime}}\left[\xi^{(i)^{\prime} \dagger}\right]$ for $\mathrm{SU}(6)$ and $A\left[A^{\dagger}\right]=\theta_{2}^{\prime}\left[\theta_{1}^{\prime}\right], B\left[B^{\dagger}\right]=\frac{1}{f^{\prime}} \xi_{n g}^{\prime}\left[\xi_{n g}^{\prime \dagger}\right]$ for $\mathrm{SU}(3)$, the above $F_{6}$-factor is given below,

$$
\begin{aligned}
F_{6}= & +\left(\theta^{\prime \dagger}+\frac{1}{f^{\prime}} \xi^{(i)^{\prime} \dagger}\right)+\left(\theta^{\prime}+\frac{1}{f^{\prime}} \xi^{(i)^{\prime}}\right)+\frac{1}{2}\left(\left[\frac{1}{f^{\prime}} \xi^{(i)^{\prime} \dagger}, \theta^{\prime}\right]-\left[\frac{1}{f^{\prime}} \xi^{(i)^{\prime}}, \theta^{\prime \dagger}\right]\right) \\
& +\frac{1}{2}\left(\left\{\theta^{\prime \dagger}, \frac{1}{f^{\prime}} \xi^{(i)^{\prime} \dagger}\right\}+\left\{\theta^{\prime}, \frac{1}{f^{\prime}} \xi^{(i)^{\prime}}\right\}\right)+\frac{1}{2}\left(\left\{\theta^{\prime \dagger}, \theta^{\prime}\right\}+\left\{\frac{1}{f^{\prime}} \xi^{(i)^{\prime} \dagger}, \frac{1}{f^{\prime}} \xi^{(i)^{\prime}}\right\}\right), \\
& i=1,2
\end{aligned}
$$

where $F_{6}$ reflects the $\mathrm{SU}(6)$ shift (symmetry) and its SS-twist symmetry breaking terms.

For $\mathrm{SU}(3)$-level, $F_{3}$ below also shows the $\mathrm{SU}(3)$ double-vacua shift symmetry and its SS-twist breaking terms, as follows,

$$
\begin{aligned}
F_{3}= & 1+\left(\theta_{i}^{\prime \dagger}+\frac{1}{f^{\prime}} \xi_{n g}^{\prime \dagger}\right)+\left(\theta^{\prime}+\frac{1}{f^{\prime}} \xi_{n g}^{\prime}\right)+\frac{1}{2}\left(\left[\frac{1}{f^{\prime}} \xi_{n g}^{\prime \dagger}, \theta_{i}^{\prime}\right]-\left[\frac{1}{f^{\prime}} \xi_{n g}^{\prime}, \theta_{i}^{\prime \dagger}\right]\right) \\
& +\frac{1}{2}\left(\left\{\theta_{i}^{\prime \dagger}, \frac{1}{f^{\prime}} \xi_{n g}^{\prime \dagger}\right\}+\left\{\theta_{i}^{\prime}, \frac{1}{f^{\prime}} \xi_{n g}^{\prime}\right\}\right)+\frac{1}{2}\left(\left\{\theta_{i}^{\prime \dagger}, \theta_{i}^{\prime}\right\}+\left\{\frac{1}{f^{\prime}} \xi_{n g}^{\dagger}, \frac{1}{f^{\prime}} \xi_{n g}^{\prime}\right\}\right), \\
& i=1,2
\end{aligned}
$$

Comparing Eq. (57), (58) and Eq. (64), also Eq. (65) the double-vacua shift symmetry is broken by the terms with commutator and anti-commutator $[17,41,44]$ which 
are called here as dimensional-deconstructing terms following Scherk-Schwarz condition for dimensional deconstruction in Eq. (4), or in short, SS-twist terms. For a special condition where NGB becomes a constant field, $\left\langle\xi^{(i)^{\prime}}\right\rangle=\left\langle\xi^{(i)^{\prime} \dagger}\right\rangle=Q_{6} \alpha_{6}$ and $\left\langle\xi_{n g}^{\prime}\right\rangle=\left\langle\xi_{n g}^{\prime \dagger}\right\rangle=Q_{3} \alpha_{3}$ for $\mathrm{SU}(6)$ and $\mathrm{SU}(3)$ successively, the commutator and anti-commutator terms of NGBs and Little Higgs-like scalars become $\frac{1}{f^{\prime}} Q_{6} \alpha_{6} \theta^{\prime}\left[\theta^{\prime \dagger}\right]$ (and $\frac{1}{f^{\prime}} Q_{3} \alpha_{3} \theta_{1}^{\prime}\left[\theta_{2}^{\prime}\right]$ ) which is the first shift symmetry breaking term in parentheses as shown in Eq. (33) and Eq. (34) too.

\subsection{The Scherk-Schwarz (SS)-twist breaking}

\subsubsection{The SU(3) unification of Higgs-NGB from SS-twist factor}

This breaking is obtained when the commutator and anti-commutator terms of NGBs and Little Higgs-like scalars achieve non-zero values. For $\mathrm{SU}(3)$ it is very clear since $\theta_{i}^{\prime} \neq \theta_{i}^{\prime \dagger}, i=1,2$ eventhough $\xi_{n g}^{\prime}=\xi_{n g}^{\prime \dagger}$ so that $\left[\frac{1}{f^{\prime}} \xi_{n g}^{\prime \dagger}, \theta_{i}^{\prime}\right] \neq\left[\frac{1}{f^{\prime}} \xi_{n g}^{\prime}, \theta_{i}^{\prime \dagger}\right]$.

In other words the commutator terms do not vanish. The same condition happens for the anti-commutator terms $\frac{1}{2}\left(\left\{\theta_{i}^{\prime \dagger}, \frac{1}{f^{\prime}} \xi_{n g}^{\prime \dagger}\right\}+\left\{\theta_{i}^{\prime}, \frac{1}{f^{\prime}} \xi_{n g}^{\prime}\right\}\right)$ which contribute to the emerging of a new scalar. Let's proceed the calculation by the following, utilizing $\xi_{n g}^{\prime}$ instead of $\xi_{n g}^{\prime \dagger}$ due to its equality,

$$
\begin{aligned}
& \left\{\theta_{1}^{\prime}, \frac{1}{f^{\prime}} \xi_{n g}^{\prime}\right\}=\frac{1}{f^{\prime 2}}\left(\begin{array}{ccc}
0 & 0 & \left(\xi^{\prime} H^{\prime}\right)_{2 \times 1} \\
0 & 0 & 0
\end{array}\right), \\
& \left\{\theta_{1}^{\prime \dagger}, \frac{1}{\left.\xi^{\prime} H^{\dagger}\right)_{1 \times 2}^{\prime}}, \quad 0=\frac{1}{f^{\prime 2}}\left(\begin{array}{ccc}
0 & 0 & \left(\xi^{\prime} H\right)_{2 \times 1} \\
0 & 0 & 0 \\
\left(\xi^{\prime} H^{\prime \dagger}\right)_{1 \times 2} & 0
\end{array}\right), \quad \theta_{1}^{\prime \dagger}=\theta_{2}^{\prime} .\right.
\end{aligned}
$$

Recalling Eq. (29) and Eq. (30) for $\theta_{1}^{\prime}$ and $\theta_{2}^{\prime}$, also Eq. (45) for $\frac{1}{f^{\prime}} \xi_{n g}^{\prime}$, the additional factors for Eq. (66) and Eq. (67) are $\left(\frac{i f_{2}^{\prime}}{f_{1}^{\prime}}\right) \times\left(-\frac{i f_{2}^{\prime}}{f_{1}^{\prime}}\right)\left[\left(\frac{i f_{1}^{\prime}}{f_{2}^{\prime}}\right)\right]=\frac{f_{2}^{\prime 2}}{f_{1}^{\prime 2}}[-1]$ and $\left(-\frac{i f_{1}^{\prime}}{f_{2}^{\prime}}\right) \times\left(-\frac{i f_{2}^{\prime}}{f_{1}^{\prime}}\right)\left[\left(\frac{i f_{1}^{\prime}}{f_{2}^{\prime}}\right)\right]=-1\left[\frac{f_{1}^{\prime 2}}{f_{2}^{\prime 2}}\right]$ so that if NGB achieves non-zero VEV Eq. (66) and Eq. (67) proportionally become $\frac{f_{2}^{\prime 2}}{f_{1}^{\prime 2}}[-1] \frac{1}{f^{\prime}}\left\langle\xi^{\prime}\right\rangle \theta_{1}^{\prime}$ and $(-1) \frac{f_{1}^{\prime 2}}{f_{2}^{\prime 2}} \frac{1}{f^{\prime}}\left\langle\xi^{\prime}\right\rangle \theta_{1}^{\prime \dagger}$ which are basically PNB Higgses.

On the other hand the commutators in Eq. (65) can be written as,

$$
\begin{aligned}
& {\left[\frac{1}{f^{\prime}} \xi_{n g}^{\prime}, \theta_{1}^{\prime}\right]=\frac{1}{f^{\prime 2}}\left(\begin{array}{ccc}
0 & 0 & \left(\xi^{\prime} H^{\prime}\right) \\
0 & 0 & \\
\left(-\xi^{\prime} H^{\dagger}\right) & 0
\end{array}\right),} \\
& {\left[\frac{1}{f^{\prime}} \xi_{n g}^{\prime}, \theta_{1}^{\prime \dagger}\right]=\frac{1}{f^{\prime 2}}\left(\begin{array}{ccc}
0 & 0 & \left(\xi^{\prime} H\right) \\
0 & 0 & \\
\left(-\xi^{\prime} H^{\prime \dagger}\right) & 0
\end{array}\right),}
\end{aligned}
$$

so that the following substracting of Eq. (68) and Eq. (69) gives the following,

$$
\left[\frac{1}{f^{\prime}} \xi_{n g}^{\prime}, \theta_{1}^{\prime}\right]-\left[\frac{1}{f^{\prime}} \xi_{n g}^{\prime}, \theta_{1}^{\prime \dagger}\right]=\frac{1}{f^{\prime 2}}\left(\begin{array}{ccc}
0 & 0 & \xi^{\prime}\left(H^{\prime}-H\right) \\
0 & 0 & 0
\end{array}\right) .
$$


Defining $H^{\prime \prime}=\left(H^{\prime}-H\right), H^{\prime \prime \dagger}=\left(H^{\prime \dagger}-H^{\dagger}\right)$ and factoring out $\xi^{\prime}$ while considering NGB $\xi^{\prime}$ achieves non-zero VEV $\left\langle\xi^{\prime}\right\rangle \neq 0$ one finds Eq. (70) producing PNB Higgs due to

$$
\left[\frac{1}{f^{\prime}} \xi_{n g}^{\prime}, \theta_{1}^{\prime}\right]-\left[\frac{1}{f^{\prime}} \xi_{n g}^{\prime}, \theta_{1}^{\prime \dagger}\right]=\frac{\left\langle\xi^{\prime}\right\rangle}{f^{\prime 2}}\left(\begin{array}{ccc}
0 & 0 & H^{\prime \prime} \\
0 & 0 & \\
H^{\prime \prime \dagger} & 0
\end{array}\right) .
$$

On the contrary NGB $\xi^{\prime}$ can also be absorbed by $H^{\prime \prime}$ to produce a new exotic scalar as well. Since $H^{\prime \prime}=\left(H^{\prime}-H\right)$ and defining $H^{\prime} \sim H$ or $\left(H^{\prime}-H\right)=\delta H \sim 0$ one can consider $\xi^{\prime} H^{\prime \prime}\left(=\xi^{\prime} \delta H\right)$ and $\xi^{\prime} H^{\prime \prime \dagger}\left(=\xi^{\prime} \delta H^{\dagger}\right)$ as new scalars $H_{H}^{\prime \prime}$ and $H_{H}^{\prime \prime \dagger}$ with dominant NGB-property and named as Scherk-Schwarz scalar type-1 or Heisenberg scalar i.e. transferring additional mass and degrees of freedom from NGBs. Here $\delta H\left(\delta H^{\dagger}\right)$ is considered as a single Higgs particle unifying with a doublet of NGBs $\xi^{\prime}$ bringing 2 (two) extra degrees of freedom which will further be clarified in subsection below.

\subsubsection{The SU(6) Unification of Higgs-NGB from SS-twist factor}

For $\mathrm{SU}(6)$ the condition is completely different due to $\xi^{(i)^{\prime}}=\xi^{(i)^{\prime} \dagger}$ and $\theta^{\prime}=\theta^{\prime \dagger}$ so that the commutator terms vanish and the anticommutator terms become a single term $\left\{\theta^{\prime}, \frac{1}{f^{\prime}} \xi^{(i)^{\prime}}\right\}$ which contributes significantly to the creation of new exotic scalars as follows,

$$
\begin{aligned}
& \left\{\frac{1}{f^{\prime}} \xi^{(1)^{\prime}}, \theta^{\prime}\right\} \\
& \left.=\frac{1}{f^{\prime 2}}\left(\begin{array}{ccc}
(0)_{3 \times 3} & \\
0 & 0 & \left(\xi_{0} H^{\prime}\right)_{2 \times 1} \\
0 & 0 & 0
\end{array}\right) \text { ( } \begin{array}{ccc}
0 & 0 & \left(\left(\xi^{\prime}+\xi_{0}\right) H\right)_{2 \times 1} \\
0 & 0 & \\
\left(\xi_{0} H^{\prime \dagger}\right)_{1 \times 2} & 0
\end{array}\right) \text {, } \\
& \left\{\frac{1}{f^{\prime}} \xi^{(2)^{\prime}}, \theta^{\prime}\right\}
\end{aligned}
$$

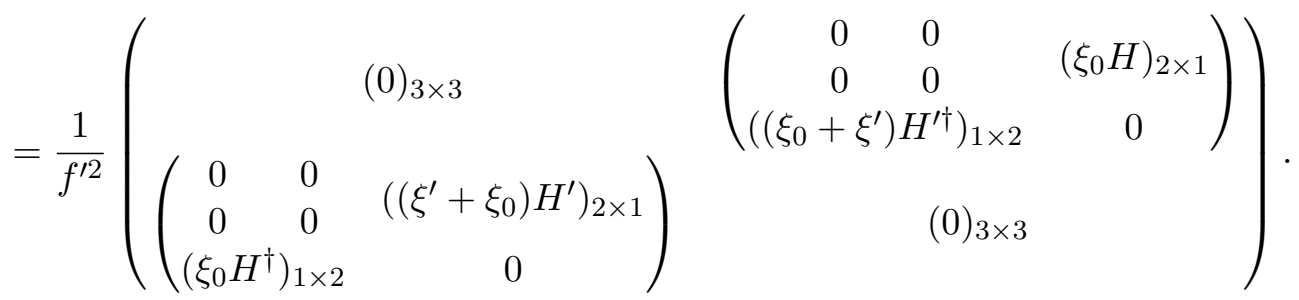

Factorizing $\xi_{0}$ out from both matrices in Eq. (72) and Eq. (73) and considering a very small non-zero VEV of $\left\langle\xi_{0}\right\rangle$ while keeping $\xi^{\prime}$ still masless following the work of J. Schwinger [17] where the massless particle can exist together with the massive particle in the strongly-coupled realm when the later is light or its VEV is small. The 
heavy massive particle $\xi_{0}$, in conjunction with the massless $\xi^{\prime}$ undergoes a breakdown or becomes unstable. Setting the following $\left\langle\xi_{0}\right\rangle \sim\left\langle H^{\prime}\right\rangle \sim\langle H\rangle \sim \mathcal{O}(100 \mathrm{GeV})$ in comparison to $\mathrm{SU}(6)$ or $\mathrm{SU}(3)$ cut off scale, $\Lambda_{(6)}^{\mathrm{ZP}} \sim \mathcal{O}(1000 \mathrm{TeV})$ and $\Lambda_{(3)} \sim$ $\mathcal{O}(100 \mathrm{TeV})$ so that $\left\langle\xi_{0}\right\rangle \rightarrow 0$, one rewrites Eq. (72) and Eq. (73) as,

$$
\begin{aligned}
& \left\{\frac{1}{f^{\prime}} \xi^{(1)^{\prime}}, \theta^{\prime}\right\}=\frac{1}{f^{\prime 2}}\left\langle\xi_{0}\right\rangle\left(\begin{array}{cccc} 
& (0)_{3 \times 3} \\
0 & 0 & \left(H^{\prime}\right) \\
0 & 0 & \\
\left(1+\frac{\xi^{\prime}}{\left\langle\xi_{0}\right\rangle}\right) H^{\dagger} & 0
\end{array}\right)
\end{aligned}
$$

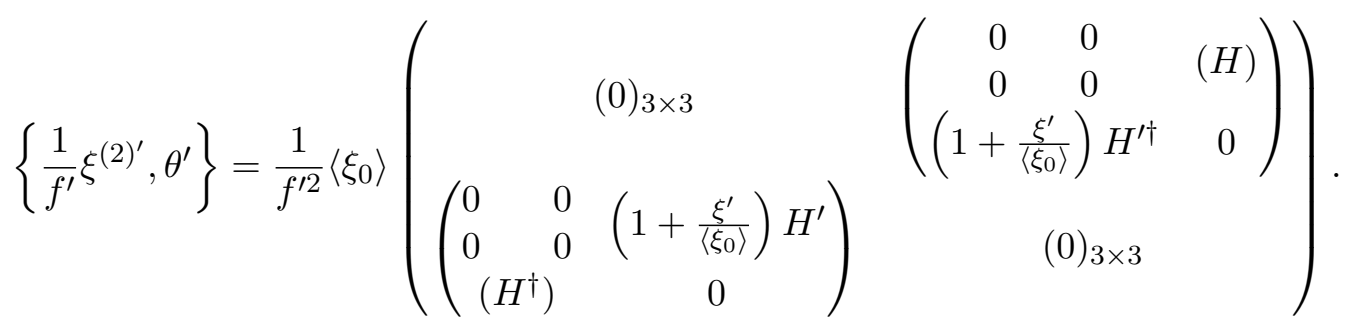

From the upper-right and lower-left $3 \times 3$ submatrices above one can easily identify the emerging of new scalars, proceeding the multiplication with $H\left[H^{\dagger}\right]$ and $H^{\prime}\left[H^{\prime \dagger}\right]$, and allowing $H$ and $H^{\prime}$ to obtain non-zero VEVs in the NGB-terms following double-vacua property in Eq. (57), as stated below,

$$
\begin{aligned}
\left(1+\frac{\xi^{\prime}}{\left\langle\xi_{0}\right\rangle}\right) H\left[H^{\dagger}\right] & \sim H\left[H^{\dagger}\right]+\frac{\langle H\rangle\left[\left\langle H^{\dagger}\right\rangle\right]}{\left\langle\xi_{0}\right\rangle} \xi^{\prime}, \\
\left(1+\frac{\xi^{\prime}}{\left\langle\xi_{0}\right\rangle}\right) H^{\prime}\left[H^{\prime \dagger}\right] & \sim H^{\prime}\left[H^{\prime \dagger}\right]+\frac{\left\langle H^{\prime}\right\rangle\left[\left\langle H^{\prime \dagger}\right\rangle\right]}{\left\langle\xi_{0}\right\rangle} \xi^{\prime},
\end{aligned}
$$

where $\frac{\langle H\rangle\left[\left\langle H^{\dagger}\right\rangle\right]}{\left\langle\xi_{0}\right\rangle} \sim \frac{\left\langle H^{\prime}\right\rangle\left[\left\langle H^{\prime \dagger}\right\rangle\right]}{\left\langle\xi_{0}\right\rangle} \sim 1$ for $\left\langle\xi_{0}\right\rangle \sim \mathcal{O}(100 \mathrm{GeV})$ so that one obtains finally the new scalar, named as Scherk-Schwarz scalar type-2A, as

$$
\begin{aligned}
& H_{0}^{\prime \prime(-)}\left[H_{0}^{\prime \prime(-) \dagger}\right]=\left(H^{\prime}\left[H^{\prime \dagger}\right]+\xi^{\prime}\right)-\left(H\left[H^{\dagger}\right]+\xi^{\prime}\right), \\
& H_{0}^{\prime \prime(+)}\left[H_{0}^{\prime \prime(+) \dagger}\right]=\left(H^{\prime}\left[H^{\prime \dagger}\right]+\xi^{\prime}\right)+\left(H\left[H^{\dagger}\right]+\xi^{\prime}\right) .
\end{aligned}
$$

Beside, considering $\left\langle\xi_{0}\right\rangle \sim \mathcal{O}(1 \mathrm{TeV}) \ll \Lambda_{(3)}<\Lambda_{(6)}^{\mathrm{ZP}}$ it is possible to define a very small positive number $\epsilon_{0}=\frac{\langle H\rangle\left[\left\langle H^{\dagger}\right\rangle\right]}{\left\langle\xi_{0}\right\rangle} \sim \frac{\left\langle H^{\prime}\right\rangle\left[\left\langle H^{\prime \dagger}\right\rangle\right]}{\left\langle\xi_{0}\right\rangle} \ll 1$ which makes Eq. (78) becoming and directly giving Scherk-Schwarz scalar type-2B as below,

$$
\begin{aligned}
& H_{0 p}^{\prime \prime(-)}\left[H_{0 p}^{\prime \prime(-) \dagger}\right]=\left(H^{\prime}\left[H^{\prime \dagger}\right]+\epsilon_{0} \xi^{\prime}\right)-\left(H\left[H^{\dagger}\right]+\epsilon_{0} \xi^{\prime}\right), \\
& H_{0 p}^{\prime \prime(+)}\left[H_{0 p}^{\prime \prime(+) \dagger}\right]=\left(H^{\prime}\left[H^{\prime \dagger}\right]+\epsilon_{0} \xi^{\prime}\right)+\left(H\left[H^{\dagger}\right]+\epsilon_{0} \xi^{\prime}\right),
\end{aligned}
$$

where both $H_{0}^{(i)^{\prime \prime}}$ and $H_{0 p}^{(i)^{\prime \prime}}, i=1,2$ are the unification of a new Higgs field $H^{\prime \prime}=$ $\left(H^{\prime}-H\right)$ or $H^{\prime \prime \dagger}=\left(H^{\prime \dagger}-H^{\dagger}\right)$ and 2 (two) NGBs. Clearly Eq. (78) and Eq. (79) 
show NGBs as the artifacts of a scalar boson by transferring some or zero mass and 2 (two) degrees of freedom. From now on, in this paper, we refer to the new gauge-like scalars as Scherk-Schwarz scalars which have 2 (two) types coming from both Eq. (78) and Eq. (79).

Comparing both equations above one can instinctively interprete with respect to PNB Higgs the influence of $\epsilon_{0}$ as being connected to the elimination of additional mass. To be clear, the first gets additional mass and 2 (two) extra degrees of freedom due to $\xi^{\prime}$ while the second only 2 (two) extra degrees of freedom due to $\epsilon_{0} \xi^{\prime}\left(\epsilon_{0} \ll 1\right)$. This property will be further justified in the next coming sections.

\section{The $\mathrm{SU}(6)$ double-vacua shift symmetry breaking un- der unitary transformation}

\subsection{The SU(6) unitarily-transformed shift symmetry breaking}

In the $5 \mathrm{D}(y \sim 0)$ near-brane, without prejudice and also without loss of generality, it is justified to require a constant Lagrangian under unitary transformations. This can be fulfilled easily if one applies trace to the matrices of potential terms since the trace is constant under such transformation [Appendix B] [45]. Let's consider the following potentials,

$$
\begin{aligned}
& V_{y g 1}^{(6)}=\lambda_{y}^{(6)} \operatorname{Tr}\left\{\left(\widetilde{\Phi}^{(i) \dagger} \Phi_{\mathrm{NG}}^{(1)}\right)\left(\Phi_{\mathrm{NG}}^{(1) \dagger} \widetilde{\Phi}^{(i)}\right)\right\}+\lambda_{y}^{(6)} \operatorname{Tr}\left\{\left(\Phi_{\mathrm{NG}}^{(1) \dagger} \widetilde{\Phi}^{(i)}\right)\left(\widetilde{\Phi}^{(i) \dagger} \Phi_{\mathrm{NG}}^{(1)}\right)\right\}, \\
& V_{y g 2}^{(6)}=\lambda_{y}^{(6)} \operatorname{Tr}\left\{\left(\widetilde{\Phi}^{(i) \dagger} \Phi_{\mathrm{NG}}^{(2)}\right)\left(\Phi_{\mathrm{NG}}^{(2) \dagger} \widetilde{\Phi}^{(i)}\right)\right\}+\lambda_{y}^{(6)} \operatorname{Tr}\left\{\left(\Phi_{\mathrm{NG}}^{(2) \dagger} \widetilde{\Phi}^{(i)}\right)\left(\widetilde{\Phi}^{(i) \dagger} \Phi_{\mathrm{NG}}^{(2)}\right)\right\},
\end{aligned}
$$

where $\widetilde{\Phi}^{(i)}$ and $\Phi_{\text {NG }}^{(j)}$ have been shown in Eq. (28) and Eq. (42). Non-zero potentials can only be obtained for $v^{T} v_{\mathrm{NG}}^{(1)}=v_{\mathrm{NG}}^{(1) T} v=f_{1}^{\prime 2}$ in Eq. (80) and $v^{\prime T} v_{\mathrm{NG}}^{(2)}=v_{\mathrm{NG}}^{(2) T} v^{\prime}=$ $f_{2}^{\prime 2}$ in Eq. (81) since $v^{T} v_{\mathrm{NG}}^{(2)}=v_{\mathrm{NG}}^{(2) T} v=v^{T} v_{\mathrm{NG}}^{(1)}=v_{\mathrm{NG}}^{(1) T} v^{\prime}=0$, in other words, $i=1$ for Eq. (80) and $i=2$ for Eq. (81). For both $\widetilde{\Phi}^{(1)}$ and $\widetilde{\Phi}^{(2)}$ the phase factor (Little Higgs-like) parameter, $\theta^{\prime}=\theta^{\prime \dagger}$, is the same while the following equality is trivial, $\operatorname{Tr}\left[\frac{1}{f^{\prime}} \xi^{(i)^{\prime} \dagger}, \theta^{\prime}\right]=\operatorname{Tr}\left[\frac{1}{f^{\prime}} \xi^{(i)^{\prime}}, \theta^{\prime \dagger}\right]$. Expanding to the lowest order and performing calculation for factor- $F_{6}$, and further taking the trace of SS-twist term which is represented by the anti-commutators in Eq. (74) and Eq. (75) one finds finally

$$
\operatorname{Tr}\left\{\frac{1}{f^{\prime}} \xi^{(1)^{\prime}}, \theta^{\prime}\right\}=\operatorname{Tr}\left\{\frac{1}{f^{\prime}} \xi^{(2)^{\prime}}, \theta^{\prime}\right\}=0 .
$$

Eq. (82) establishes double-vacua shift symmetry where $F_{6}$ factor, after the vanishing of the commutator and anti-commutator, becomes as follows,

$$
\begin{aligned}
F_{6}= & +\left(\theta^{\prime \dagger}+\frac{1}{f^{\prime}} \xi^{(i)^{\prime} \dagger}\right)+\left(\theta^{\prime}+\frac{1}{f^{\prime}} \xi^{(i)^{\prime}}\right)+\frac{1}{2}\left(\left\{\theta^{\prime \dagger}, \theta^{\prime}\right\}+\left\{\frac{1}{f^{\prime}} \xi^{(i)^{\prime} \dagger}, \frac{1}{f^{\prime}} \xi^{(i)^{\prime}}\right\}\right), \\
& i=1,2
\end{aligned}
$$


which means double-vacua shift symmetry is broken if either Little Higgs-like scalar or NGB gets its mass. Comparing to Eq. (33) and Eq. (34) this is exactly the asymptotic shift symmetry breaking due to significant factor $\frac{1}{f^{\prime 2}}$.

The vanishing of SS-twist under unitary transformation of SU(6) with constant Coleman-Weinberg potential as shown in Eq. (83) cancels out the emerging of Scherk-Schwarz scalar from SS-twist as clearly demonstrated by Eq. (74) to Eq. (79). The elimination of Scherk-Schwarz scalar type-I in SU(6) is automatic due to equal traces of commutators while Scherk-Schwarz scalar type-II due to zero value of the traces of anti commutators.

\subsection{The shiftedly-and twistedly-broken Scherk-Schwarz scalar un- der unitary transformation}

\subsubsection{The shiftedly-broken Scherk-Schwarz scalar from SU(6) shift sym- metry breaking}

Setting the breaking term (anti-commutators in $F_{6}$ ) in Eq. (64) to zero the doublevacua shift symmetry shows up as,

$$
\theta^{\prime \dagger}\left(\frac{1}{f^{\prime}} \xi^{(i)^{\prime} \dagger}\right)\left[\text { or } \theta^{\prime}\left(\frac{1}{f^{\prime}} \xi^{(i)^{\prime}}\right)\right] \longrightarrow \theta^{\prime \dagger}\left[\theta^{\prime}\right]+\frac{1}{f^{\prime}} \xi^{(i)^{\prime} \dagger}\left[\frac{1}{f^{\prime}} \xi^{(i)^{\prime}}\right]
$$

which preserves another double-vacua shift $H^{\prime}[H]+\xi^{\prime}$ and $H^{\prime}[H]+\epsilon \xi^{\prime}$. Despite no massive Scherk-Schwarz scalar can show up in SU(6) due to constraint in Eq. (82) but Eq. (84) fortunately preserves the existence of the new scalars, $H_{0}^{\prime \prime}$ and $H_{0 p}^{\prime \prime}$, in Eq. (78) and Eq. (79). This means that after SU(6) symmetry breaking into $\mathrm{SU}(3)$ the new gauge-like scalars can show up in the massive forms, considering the doublet states of $H^{\prime}, H$ and $\xi^{\prime}$, after significant shift breaking (terms) facilitated by the anti-commutators of $\left\{\theta^{\prime \dagger}, \theta^{\prime}\right\}$ and $\left\{\frac{1}{f^{\prime}} \xi^{(i)^{\prime} \dagger}, \frac{1}{f^{\prime}} \xi^{(i)^{\prime}}\right\}$ in Eq. (83) which are due to strongly-coupled Little Higgs-like scalar and NGB [3]. The emerging massive gaugelike scalar must have been facilitated by the existence of SU(3) SS-twist produced by $\mathrm{SU}(6)$ shift symmetry breaking prior to which the gauge-like scalar remains massless.

Since $H_{0}^{\prime \prime} \neq H_{H}^{\prime \prime}$ and $H_{0 p}^{\prime \prime} \neq H_{H}^{\prime \prime}$ the SS-twist, in this case, must be different with $\mathrm{SU}(3)$-origin SS-twist discussed in subsection 4.2.1. which means that it must originate from the entity composed by $H_{0}^{\prime \prime}\left[H_{0 p}^{\prime \prime}\right]$ and $H_{0}^{\prime \prime \dagger}\left[H_{0 p}^{\prime \prime \dagger}\right]$ i.e. SS-twist is contained inclusively in the mass term $H_{0}^{\prime \prime \dagger} H_{0}^{\prime \prime}\left[H_{0 p}^{\prime \prime \dagger} H_{0 p}^{\prime \prime}\right]$ as clearly shown below.

Taking $H_{0}^{\prime \prime}\left[H_{0 p}^{\prime \prime}\right]$ as a massless gauge-like scalar with 2 (two) degrees of freedom (in the $\hat{i}$ and $\hat{j}$ direction) and rewriting Eq. (78) and Eq. (79) as,

$$
\begin{aligned}
H_{0}^{\prime \prime}\left[H_{0}^{\prime \prime \dagger}\right] & =\left(H^{\prime}\left[H^{\prime \dagger}\right]+\xi^{\prime}\right) \hat{i}-\left(H\left[H^{\dagger}\right]+\xi^{\prime}\right) \hat{j}, \\
H_{0 p}^{\prime \prime}\left[H_{0 p}^{\prime \prime \dagger}\right] & =\left(H^{\prime}\left[H^{\prime \dagger}\right]+\epsilon \xi^{\prime}\right) \hat{i}-\left(H\left[H^{\dagger}\right]+\epsilon \xi^{\prime}\right) \hat{j},
\end{aligned}
$$

where $\epsilon$ is a very small positive number for unitarily-transformed Scherk-Schwarz 
scalar, one finds the mass term as,

$$
\begin{aligned}
& \operatorname{Tr}\left(H_{0}^{\prime \prime \dagger} H_{0}^{\prime \prime}\right)=\operatorname{Tr}\left\{\left(H^{\prime \dagger}+\xi^{\prime}\right)\left(H^{\prime}+\xi^{\prime}\right)\right\}+\operatorname{Tr}\left\{\left(H^{\dagger}+\xi^{\prime}\right)\left(H+\xi^{\prime}\right)\right\}, \\
& \operatorname{Tr}\left(H_{0 p}^{\prime \prime \dagger} H_{0 p}^{\prime \prime}\right)=\operatorname{Tr}\left\{\left(H^{\prime \dagger}+\epsilon \xi^{\prime}\right)\left(H^{\prime}+\epsilon \xi^{\prime}\right)\right\}+\operatorname{Tr}\left\{\left(H^{\dagger}+\epsilon \xi^{\prime}\right)\left(H+\epsilon \xi^{\prime}\right)\right\} .
\end{aligned}
$$

Making use the following approach: $H^{\prime} \sim H$ and $\left(H^{\prime} \xi-H \xi\right) \sim 0$ followed by adding it to Eq. (87) while similar approach with the substitution $\xi \rightarrow \epsilon \xi$ is performed prior to its adding to Eq. (88). Finally both Eq. (87) and Eq. (88) can be expressed with inclusive SS-twist terms as follows,

$$
\operatorname{Tr}\left(H_{0}^{\prime \prime \dagger} H_{0}^{\prime \prime}\right)=\left(H^{\prime \dagger} H^{\prime}+H^{\dagger} H\right)+\operatorname{Tr}\left(\left\{\xi^{\prime}, H^{\prime}\right\}+\left\{\xi^{\prime}, H^{\prime \dagger}\right\}\right)+\operatorname{Tr}\left(\left[\xi^{\prime}, H\right]-\left[\xi^{\prime}, H^{\dagger}\right]\right),
$$

with similar expression for $\operatorname{Tr}\left(H_{0}^{\prime \prime \dagger} H_{0}^{\prime \prime}\right)$ obtained after substituting $\xi \rightarrow \epsilon \xi^{\prime}$. Eq. (89) shows clearly the mass contribution and transfer of degrees of freedom of NGBs via SS-twist term to PNB Higgs for $H_{0}^{\prime \prime}$ while for $H_{o p}^{\prime \prime}$ only degrees of freedom of NGBs to PNB Higgs due to $\epsilon \rightarrow 0$. This has confirmed the existence of 2 (two) types of Scherk-Schwarz scalars in SU(3) level with the masses larger than and equal to the PNB Higgs mass for $H_{0}^{\prime \prime}$ and $H_{0 p}^{\prime \prime}$ successively.

A special phenomenon takes place in Eq. (89) where the $H_{0}^{\prime \prime}$-mass term is decomposed into 2 (two) Higgses mass terms, $H^{\prime}$ - and $H$-mass terms, with 2 (two) NGBs in SS-twist term indicating the breaking of a massive type-II A Scherk-Schwarz scalar into 2 (two) SM Higgses with NGBs decoupling into extra-dimensional 5D SS-twist.

The result above i.e. the stable $H_{0 p}^{\prime \prime}$ and the unstable $H_{0}^{\prime \prime}$ confirm convincingly the longtime-age work of J. Schwinger [17] stating that the massless boson can not exist together with the massive boson which has a large mass in the strongly-intercated realm otherwise the later becomes unstable and breaks. The unified Higgs-NGB can take on stable form if the massive Higgs is relatively light. Therefore $\mathrm{SU}(6)$-origin $H_{0}^{\prime \prime}$ becomes unstable but $\mathrm{SU}(3)$-origin $H_{H}^{\prime \prime}$ be stable.

Now, let's start the analysis to establish the merging scalars and its masses from $\mathrm{SU}(3)$-origin and SU(6)-origin Scherk-Schwarz scalars in the next section.

\subsubsection{The twistedly-broken Scherk-Schwarz scalar as the origin of SU(6) exotic scalar under infinitesimal unitary transformation}

This is the situation where the traces in Eq. (82) achieve non-zero values or no trace can be taken due to undefined matrix multiplication. The second condition is accomplished by the so-called triplet-triplet splitting prior to SU(6) shift symmetry breaking, in other words, the splitting is triggered by the contradicting weaklystrongly coupled condition of 2 (two) triplets in one sextet in the same way as tripletdoublet splitting of SU(5) Higgs quintet [48]. In other words, $\phi^{(i)}$ separates itself from $\phi_{0}^{(i)}$ in the sextet of will-be-SimplestLittleHiggs scalar in Eq. (23) becoming Eq. (25) and Eq. (26) accordingly.

Now, one is going further by questioning what really is behind the separating weakly-strongly coupled triplets from a sextet which must be connected to stronglyapproach in Eq. (24). Before analyzing the exponent matrix in Eq. (24) in this 
discussion of unitarily-transformed sextet one defines hereby the infinitesimal unitary transformation [7] under which trace of scalar product (or power- $n$ ) is constant [45] which can be expressed, in general, as follows,

$$
U=1+i \epsilon G
$$

where $\epsilon=\frac{1}{f^{\prime}}$ and $G$ is a hermitian operator which, in this case, is set equal to phase-factor matrix representation of NGB $\xi^{\prime}$ and $U$ unitary transformation so that $U=1+i \frac{1}{f^{\prime}} \xi^{\prime}$. The transformation provides some basic properties such as $U U^{\dagger}=$ $U^{\dagger} U=I\left(I_{6}\right.$ for $\mathrm{SU}(6), I_{3}$ for $\left.\mathrm{SU}(3)\right), \widetilde{\Phi}^{(i)} \rightarrow \widetilde{\Phi}^{(i)^{\prime \prime}}=U \widetilde{\Phi}^{(i)} U^{\dagger}, i=1,2$ from which the following identities are obtained,

$$
\widetilde{\Phi}^{(i)^{\prime \prime} \dagger} \widetilde{\Phi}^{(i)^{\prime \prime}}=U\left(\widetilde{\Phi}^{(i) \dagger} \widetilde{\Phi}^{(i)}\right) U^{\dagger}, \quad \widetilde{\Phi}^{(i) \dagger} \widetilde{\Phi}^{(i)}=U^{\dagger}\left(\widetilde{\Phi}^{(i)^{\prime \prime} \dagger} \widetilde{\Phi}^{(i)^{\prime \prime}}\right) U,
$$

where Eq. (91) provides the equality as below,

$$
\operatorname{Tr}\left(\widetilde{\Phi}^{(i)^{\prime \prime} \dagger} \widetilde{\Phi}^{(i)^{\prime \prime}}\right)=\operatorname{Tr}\left(\widetilde{\Phi}^{(i) \dagger} \widetilde{\Phi}^{(i)}\right)=C,
$$

under the condition where $C$ is a constant, that is, if scalar product attains constancy due to its trace is constant [45]. For simplicity we neglect temporarily the ratio $\frac{f_{2}^{\prime}}{f_{1}^{\prime}}\left[\frac{f_{1}^{\prime}}{f_{2}^{\prime}}\right]$ and rewrite

$$
\widetilde{\Phi}^{(1)}\left[\widetilde{\Phi}^{(2)}\right]=\widetilde{\Phi}_{0}^{(1)}\left[\widetilde{\Phi}_{0}^{(2)}\right] v\left[v^{\prime}\right], \quad \widetilde{\Phi}_{0}^{(1)}\left[\widetilde{\Phi}_{0}^{(2)}\right]=e^{\theta}\left[e^{-\theta}\right],
$$

where $\operatorname{Tr} \widetilde{\Phi}^{(1) \dagger} \widetilde{\Phi}^{(1)}=C=v^{2}$ and $\operatorname{Tr} \widetilde{\Phi}^{(2) \dagger} \widetilde{\Phi}^{(2)}=C=v^{\prime 2}$ or $C= \begin{cases}v^{2}, & i=1 ; \\ v^{\prime 2}, & i=2 .\end{cases}$

This is exactly the case of Little Higgs-like scalar $\widetilde{\Phi}^{(i)}, i=1,2$ which produces PNB Higgs, later on after the triplet-triplet splitting in $\mathrm{SU}(3)$ symmetry breaking due to $C=\left(\phi_{0}^{(i) \dagger} \phi_{0}^{(i)}\right)+\left(\phi^{(i) \dagger} \phi^{(i)}\right)$ following Eq. (23) which can further be written as,

$$
C=f_{i}^{\prime 2}+f_{i}^{\prime 2} e^{i\left(\theta_{i}^{\prime}-\theta_{j}^{\prime}\right)} \sim f_{i}^{\prime 2}\left(2 \pm \frac{1}{2} v^{\prime \prime 2} \pm H^{\prime \prime \dagger} H^{\prime \prime}\right), i \neq j=1,2,
$$

where $\operatorname{Tr}\left(H^{\prime \prime} H^{\prime \prime \dagger}\right)=\frac{1}{2} v^{\prime \prime 2}, H^{\prime \prime}=\left(H^{\prime}-H\right)$, $v^{\prime \prime}$ electroweak scale, $\sim \mathcal{O}(100 \mathrm{GeV})$.

Any deviation from the above equality in Eq. (92) means the emerging of exoticity with respect to Little Higgs-like scalar due to the existence of transformation $U$ (or NGB $\xi^{(i)^{\prime}}$ influence).

In general $\widetilde{\Phi}^{(i)^{\prime \prime}} \neq \widetilde{\Phi}^{(i)}$ due to Eq. (90) to Eq. (91) which can be expressed in the following,

$$
\begin{aligned}
\widetilde{\Phi}^{(i)^{\prime \prime} \dagger} \widetilde{\Phi}^{(i)^{\prime \prime}}= & \widetilde{\Phi}^{(i) \dagger} \widetilde{\Phi}^{(i)}+i \epsilon\left(\widetilde{\Phi}^{(i) \dagger}\left[G, \widetilde{\Phi}^{(i)}\right]+\left[G, \widetilde{\Phi}^{(i) \dagger}\right] \widetilde{\Phi}^{(i)}\right)-\epsilon^{2}\left[G, \widetilde{\Phi}^{(i) \dagger}\right]\left[G, \widetilde{\Phi}^{(i)}\right], \\
& i=1,2 .
\end{aligned}
$$

From Eq. (92) the above scalar product equality of Little Higgs-like scalar can immediately be obtained if the second and third terms of the righthand side of 
Eq. (95) attaining zero value. Hence, the non-zero second (third) term defines clearly the exoticity property of Little Higgs-like scalar or $\widetilde{\Phi}^{(i)^{\prime \prime}}$ as the emerging exotic scalar and it is called the exoticity term. Let's verify the value of the second term by expanding $\widetilde{\Phi}^{(i)}\left[\widetilde{\Phi}^{(i) \dagger}\right], i=1,2$ in the strong approach such as shown in Eq. (24). Recalling the constant $C$ of scalar product one obtains immediately,

$$
\begin{aligned}
\operatorname{Tr}\left(\widetilde{\Phi}^{(i) \dagger}\left[G, \widetilde{\Phi}^{(i)}\right]\right) & =(4-2 C) \xi^{\prime}+(6-3 C) \xi_{0}, \\
\operatorname{Tr}\left(\left[G, \widetilde{\Phi}^{(i) \dagger}\right], \widetilde{\Phi}^{(i)}\right) & =(2 C-4) \xi^{\prime}+(3 C-6) \xi_{0}, i=1,2
\end{aligned}
$$

for $G=\xi^{(1)^{\prime}}$ while for $G=\xi^{(2)^{\prime}}$ one just exchanges $\xi^{\prime} \leftrightarrow \xi_{0}$ in both Eq. (96) and Eq. (97) which sum up to zero. This causes the vanishing of the second term and leaving the third term as the exoticity term. It is clear, in upper-near-brane $\epsilon=\frac{1}{f^{\prime}} \rightarrow 0$ due to high values of $f_{i}^{\prime}, i=1,2$ but $\epsilon$ becomes significant in the lowernear-brane which triggers the asymptotically-twisted shift symmetry breaking as shown in Eq. (95).

In this case the term $\left[G, \widetilde{\Phi}^{(i) \dagger}\right]\left[G, \widetilde{\Phi}^{(i)}\right], i=1,2$ where $G=\xi^{(j)^{\prime}}, j=1,2$, contains the scalar exoticity property which is realized as transferring of NGB-contributed additional mass and 2 (two) degrees of freedom to PNB Higgs so that transforming it into a gauge-like scalar. This process is realized through triplet-triplet splitting as discussed briefly below.

\subsubsection{The transferring of exoticity property (swallowing-digesting mode) in the unification of PNB Higgs-NGB}

Firstly, one proceeds the calculation of $\left[\xi^{(j)}, \widetilde{\Phi}^{(i)}\right]$ and $\left[\xi^{(j)}, \widetilde{\Phi}^{(i) \dagger}\right]$ by applying the strong approach as shown by Eq. (24) for $\widetilde{\Phi}^{(i)}\left[\widetilde{\Phi}^{(i) \dagger}\right]$ and simplifying by neglecting the figure $\frac{f_{2}^{\prime}}{f_{1}^{\prime}}\left[\frac{f_{1}^{\prime}}{f_{2}^{\prime}}\right]$ since it does not affect the result. For $G=\xi^{\prime(1)}$ the commutators become as follows,

$$
\begin{aligned}
{\left[\xi^{(1)^{\prime}}, \widetilde{\Phi}^{(i)}\right] } & =\left(\begin{array}{cc}
(0)_{3 \times 3} & \left(\xi_{n g}^{\prime} e^{i[-i] \theta_{2}^{\prime}}-e^{i[-i] \theta_{2}^{\prime}} \xi_{0}^{\prime}\right) \\
\left(\xi_{0}^{\prime} e^{i[-i] \theta_{1}^{\prime}}-e^{i[-i] \theta_{1}^{\prime}} \xi_{n g}^{\prime}\right) & (0)_{3 \times 3}
\end{array}\right), \\
{\left[\xi^{(1)^{\prime}}, \widetilde{\Phi}^{(i) \dagger}\right] } & =\left(\begin{array}{cc}
(0)_{3 \times 3} & \left(\xi_{n g}^{\prime} e^{-i[i] \theta_{1}^{\prime}}-e^{-i[i] \theta_{1}^{\prime}} \xi_{0}^{\prime}\right) \\
\left(\xi_{0}^{\prime} e^{-i[i] \theta_{2}^{\prime}}-e^{-i[i] \theta_{2}^{\prime}} \xi_{n g}^{\prime}\right) & (0)_{3 \times 3}
\end{array}\right),
\end{aligned}
$$

which bring directly to the following trace,

$$
\operatorname{Tr}\left[\xi^{(1)^{\prime}}, \widetilde{\Phi}^{(i) \dagger}\right]\left[\xi^{(1)^{\prime}}, \widetilde{\Phi}^{(i)}\right]=2 \operatorname{Tr}\left(\xi_{0}^{\prime} \xi_{n g}^{\prime}-\xi_{0}^{\prime 2}-\xi_{n g}^{\prime 2}+\xi_{n g}^{\prime} \xi_{0}^{\prime}\right) .
$$

Proceeding with $G=\xi^{(2)^{\prime}}$ one exchanges $\xi_{n g}^{\prime} \leftrightarrow \xi_{0}^{\prime}$ which also gives the same result as shown in Eq. (100) so that, in general, one can write the following result, after applying $\operatorname{Tr}\left(\xi_{n g}^{\prime} \xi_{0}^{\prime}\right)=\operatorname{Tr}\left(\xi_{0}^{\prime} \xi_{n g}^{\prime}\right)=2 \xi^{\prime} \xi_{0}, \operatorname{Tr}\left(\xi_{n g}^{\prime 2}\right)=2 \xi^{\prime 2}, \operatorname{Tr}\left(\xi_{0}^{\prime 2}\right)=3 \xi_{0}^{2}, \epsilon=\frac{1}{f^{\prime}}$,

$$
\operatorname{Tr}\left[\xi^{(j)^{\prime}}, \widetilde{\Phi}^{(i) \dagger}\right]\left[\xi^{(j)^{\prime}}, \widetilde{\Phi}^{(i)}\right]=2\left(4 \xi^{\prime} \xi_{0}-2 \xi^{\prime 2}-3 \xi_{0}^{2}\right), i, j=1,2 .
$$


Following the same setting in subsection 4.2.2. where NGB $\xi_{0}$ achieves non-zero $\mathrm{VEV}\left\langle\xi_{0}\right\rangle$ in the double-vacua one can rewrite Eq. (100), neglecting a constant, as

$$
\operatorname{Tr}\left[\xi^{(j)^{\prime}}, \widetilde{\Phi}^{(i) \dagger}\right]\left[\xi^{(j)^{\prime}}, \widetilde{\Phi}^{(i)}\right]=2\left\langle\xi_{0}^{2}\right\rangle\left(\frac{4 \xi^{\prime}}{\left\langle\xi_{0}\right\rangle}-\frac{2 \xi^{\prime 2}}{\left\langle\xi_{0}^{2}\right\rangle}\right),
$$

where the righthand side of Eq. (102) depicts the transfer of and contribution of (NGB) exoticity property to PNB Higgs. In this case the first term contributes for 2 (two) extra degrees of freedom for each new Higgs field and the second for the additional mass to each new Higgs field as clearly shown by the ratio 2:1 between the massless $\xi^{\prime}$ and the mass term $\xi^{\prime 2}\left(=\xi^{\prime \dagger} \xi^{\prime}\right)$. Special condition emerges when $\frac{1}{\left\langle\xi_{0}^{2}\right\rangle} \sim 0$ which means NGB only transfers 2 (two) extra degrees of freedom without adding any mass to PNB Higgs. This property is shown and realized through the following further details. Again, from Eq. (102) one can easily predicts the existence of 2 (two) types of gauge-like Scherk-Schwarz scalars. The first is heavier than PNB Higgs while the second has the same mass with respect to (dual to) PNB Higgs.

This can be made clear in the following way with the aid of Eq. (101), one rewrites Eq. (95) after dividing with $\epsilon^{2}$ and the vanishing of $i \epsilon$-term as the representation of PNB Higgs-NGB unification, after taking trace as below,

$$
f^{\prime 2} \operatorname{Tr}\left(\widetilde{\Phi}^{(i)^{\prime \prime} \dagger} \widetilde{\Phi}^{(i)^{\prime \prime}}\right)=f^{\prime 2} \operatorname{Tr}\left(\widetilde{\Phi}^{(i) \dagger} \widetilde{\Phi}^{(i)}\right)-\left(4\left\langle\xi_{0}^{2}\right\rangle\right)\left(\frac{1}{\left\langle\xi_{0}\right\rangle} 2 \xi^{\prime}-\frac{1}{\left\langle\xi_{0}^{2}\right\rangle} \xi^{\prime \dagger} \xi^{\prime}\right),
$$

where $\left\langle\xi_{0}\right\rangle \sim \mathcal{O}(100 \mathrm{GeV}$ and $1 \mathrm{TeV})$. To be more distinctive let's set $\left\langle\xi_{0}\right\rangle=f^{\prime} \sim$ $\mathcal{O}(1 \mathrm{TeV})$ and $\left\langle\xi_{0}\right\rangle=v^{\prime \prime} \sim \mathcal{O}(100 \mathrm{GeV})$ as before so that Eq. (103) can be reexpressed as follows,

$$
\begin{aligned}
& \operatorname{Tr}\left(\widetilde{\Phi}^{(i)^{\prime \prime} \dagger} \widetilde{\Phi}^{(i)^{\prime \prime}}\right)-\operatorname{Tr}\left(\widetilde{\Phi}^{(i) \dagger} \widetilde{\Phi}^{(i)}\right)=4\left(\frac{1}{f^{\prime 2}} \xi^{\prime \dagger} \xi^{\prime}-\frac{1}{f^{\prime}} 2 \xi^{\prime}\right), \\
& \operatorname{Tr}\left(\widetilde{\Phi}^{(i)^{\prime \prime} \dagger} \widetilde{\Phi}^{(i)^{\prime \prime}}\right)-\operatorname{Tr}\left(\widetilde{\Phi}^{(i) \dagger} \widetilde{\Phi}^{(i)}\right)=4\left(\frac{1}{f^{\prime 2}} \xi^{\prime \dagger} \xi^{\prime}-\frac{v^{\prime \prime}}{f^{\prime}} \frac{1}{f^{\prime}} 2 \xi^{\prime}\right),
\end{aligned}
$$

where $\mathcal{O}\left(\frac{v^{\prime \prime}}{f^{\prime}}\right) \sim \mathcal{O}\left(10^{-1}\right)$ and $\left(\frac{v^{\prime \prime}}{f^{\prime}}\right) \frac{1}{f^{\prime}}\left(2 \xi^{\prime}\right)$ vanishes. One has neglected this tiny mass of NGB but, of course, still considered the swallowing effect of massless NGBs in the above Eq. (105). For exotic scalars $\widetilde{\Phi}^{(i)^{\prime \prime}}$, exoticity property comes solely from NGB as shown clearly by Eq. (104) and Eq. (105) through unification process with (without) additional mass and with additional degrees of freedom under twistedlybroken shift symmetry breaking in the unitary transformation.

The first is applicable for the transferring of exoticity property consisting of 2 (two) degrees of freedom and additional mass to PNB Higgs while the second excludes the additional mass $\left(H_{0 p}^{\prime \prime}\right)$. The digesting term $\left(\frac{v^{\prime \prime}}{f^{\prime}}\right) \frac{1}{f^{\prime}}\left(2 \xi^{\prime}\right)$ must be responsible for mass contribution to a gauge-like scalar since the digested NGB can not stay alone but coupled to (and digested by) PNB Higgs which also receives 2 (two) extra degrees of freedom form $\frac{1}{f^{\prime 2}} \xi^{\prime \dagger} \xi^{\prime}$.

Eq. (103) has provided two types of exoticity terms i.e. the first consists of $\left(\frac{1}{\left\langle\xi_{0}\right\rangle} 2 \xi^{\prime}\right)-\left(\frac{1}{\left\langle\xi_{0}^{2}\right\rangle} \xi^{\prime \dagger} \xi^{\prime}\right)$ and the second of $\left(-\frac{1}{\left\langle\xi_{0}^{2}\right\rangle} \xi^{\prime \dagger} \xi^{\prime}\right)$ which one calls here as the 
digesting mode and the swallowing mode respectively. In swallowing mode no additional mass involves while in the digesting mode the addition of mass takes place.

Now, one can define the swallowing mode as the transferring of 2 (two) degrees of freedom from 2 (two) NGBs without mass transfer, indicated by $\left(-\frac{1}{\left\langle\xi_{0}^{2}\right\rangle} \xi^{\prime \dagger} \xi^{\prime}\right)$, which can be regarded as being massless. On the other side the digesting mode consists of the transferring of 2 (two) degrees of freedom and additional mass by, and as shown by, the term $\frac{1}{\left\langle\xi_{0}\right\rangle} 2\left(\xi^{\prime}\right)$, where $\left(2 \xi^{\prime}\right)$ vanishes after the coupling with and additional mass is obtained by, PNB Higgs.

\subsubsection{The swallowing-digesting mode in the triplet-triplet splitting}

Recalling Eq. (96) and Eq. (97) the vanishing of the $i \epsilon$-term is due to the constant scalar product in Eq. (92). Nevertheless non-zero VEV which brings the condition $\left\langle\widetilde{\Phi}^{(i) \dagger}\right\rangle=v^{T}\left[v^{\prime T}\right]$ and $\left\langle\widetilde{\Phi}^{(i)}\right\rangle=v\left[v^{\prime}\right]$ can happen in one single vacuum of a parallel double-vacua system causing the $i \epsilon$-term not vanishing any longer. This also happens in an inverted double-vacua system where the following condition takes place $\left\langle\widetilde{\Phi}^{(i) \dagger}\right\rangle=v^{T}\left[v^{T}\right]$ and $\left\langle\widetilde{\Phi}^{(i)}\right\rangle=v^{\prime}[v]$. Both conditions give different scalar triplets in different modes of unification but trigger the same triplet-triplet splitting.

To see the relation with triplet-triplet splitting in Eq. (23) and Eq. (46), Eq. (98) and Eq. (99) are rewritten again taking into account factor $\frac{f_{2}^{\prime}}{f_{1}^{\prime}}\left[\frac{f_{1}^{\prime}}{f_{2}^{\prime}}\right]$ of $\theta_{1}^{\prime}\left[\theta_{2}^{\prime}\right]$ followed by multiplication with $\operatorname{VEV} v\left(v^{\prime}\right)$ or $v^{T}\left(v^{\prime T}\right)$ with respect to $\widetilde{\Phi}_{0}^{(i)}, i=1,2$ for both commutators, taking first $i=1$, as follows

$$
\left[\xi^{(1)^{\prime}}, \widetilde{\Phi}_{0}^{(1)}\right] v=\left(\left(\xi_{0}^{\prime} e^{\frac{i f_{2}^{\prime}}{f_{1}^{\prime}}\left[-\frac{i f_{1}^{\prime}}{f_{2}^{\prime}}\right] \theta_{1}^{\prime}}-e^{\frac{i f_{2}^{\prime}}{f_{1}^{\prime}}\left[-\frac{i f_{1}^{\prime}}{f_{2}^{\prime}}\right]} \theta_{1}^{\prime} \xi_{n g}^{\prime}\right)\left(\begin{array}{c}
0 \\
0 \\
f_{1}^{\prime}
\end{array}\right)\right)
$$

Making use SimplestLittleHiggs-like scalar in Eq. (26) one rewrites again Eq. (106) in short form as,

$$
\left[\xi^{(1)^{\prime}}, \widetilde{\Phi}_{0}^{(1)}\right] v=\left(\xi_{0} \phi^{(1)}\left[e^{-\frac{i f_{3 \times 1}^{\prime}}{f_{2}^{\prime}} \theta_{1}^{\prime}}\left(\begin{array}{c}
0 \\
0 \\
f_{1}^{\prime}
\end{array}\right)\right]\right)
$$

taking into account $\xi_{0}^{\prime}\left(\begin{array}{c}0 \\ 0 \\ f_{1}^{\prime}\end{array}\right)=\xi_{0}\left(\begin{array}{c}0 \\ 0 \\ f_{1}^{\prime}\end{array}\right)$ and $\xi_{n g}^{\prime}\left(\begin{array}{c}0 \\ 0 \\ f_{1}^{\prime}\end{array}\right)=\left(\begin{array}{lll}0 & 0 & f_{1}^{\prime}\end{array}\right) \xi_{n g}^{\prime}=0$. Subsequently one rewrites Eq. (107) by separating $\phi^{(1)}$ from $e^{-\frac{i f_{1}^{\prime}}{f_{2}^{\prime}} \theta_{1}^{\prime}}\left(\begin{array}{c}0 \\ 0 \\ f_{1}^{\prime}\end{array}\right)$ which gives the following result,

$$
\left[\xi^{(1)^{\prime}}, \widetilde{\Phi}_{0}^{(1)}\right] v=\left(\begin{array}{c}
(0)_{3 \times 1} \\
\xi_{0} \phi^{(1)}
\end{array}\right)\left[\left(\xi_{0} e^{-\frac{(0)_{3 \times 1}^{\prime}}{f_{2}^{\prime}} \theta_{1}^{\prime}}\left(\begin{array}{c}
0 \\
0 \\
f_{1}^{\prime}
\end{array}\right)\right)\right]
$$


the first term (in front) represents PNB Higgs after factoring out $\xi_{0}$ when NGB $\xi_{0}$ achieves non-zero $\mathrm{VEV},\left\langle\xi_{0}\right\rangle \neq 0$, and consequently, the second (in bracket) reflects its degenerate Scherk-Schwarz scalar i.e the one without additional mass, and referred to as the dual of PNB Higgs.

Analogous to Eq. (108) one replaces $\xi^{(1)^{\prime}}$ with $\xi^{(2)^{\prime}}$ and also proceeds again for $i=2$ for both $\xi^{(1)^{\prime}}$ and $\xi^{(2)^{\prime}}$ successively which gives the other PNB Higgs and its dual. The other VEV-commutators are given below,

$$
\begin{aligned}
& {\left[\xi^{(2)^{\prime}}, \widetilde{\Phi}_{0}^{(1)}\right] v=\left(i[-i]\left(\begin{array}{c}
\left(f_{1}^{\prime} \xi^{\prime} H^{\prime}\right) \\
0
\end{array}\right)-\xi_{0} \phi^{(1)}\left[e^{-\frac{i f_{1}^{\prime}}{f_{2}^{\prime}} \theta_{1}^{\prime}}\left(\begin{array}{c}
0 \\
0 \\
f_{1}^{\prime}
\end{array}\right)\right]\right),}
\end{aligned}
$$

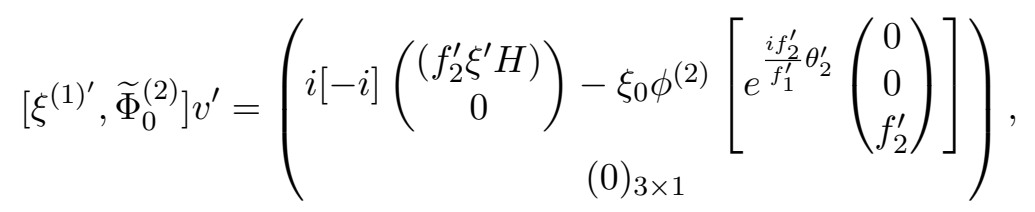

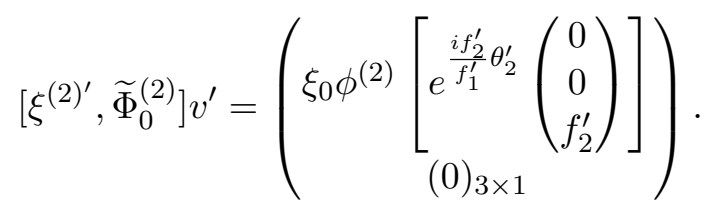

From Eq. (109) to Eq. (111) the sextets are contracted immediately into triplets when SU(6) symmetry breaking takes place. A linear combination of two VEVcommutators suffers accordingly from triplet-triplet splitting when $\mathrm{SU}(6)$ symmetry breaks. Let's analyze further what can be given by these VEV-commutators. The combinations of $\xi^{(1)^{\prime}}$ and $\xi^{(2)^{\prime}}$ with $\widetilde{\Phi}_{0}^{(i)}, i=1,2$ for VEVs $v$ and $v^{\prime}$ respectively provide directly the results as shown by the following,

$$
\begin{aligned}
& {\left[\xi^{(1)^{\prime}}, \widetilde{\Phi}_{0}^{(1)}\right] v+\left[\xi^{(2)^{\prime}}, \widetilde{\Phi}_{0}^{(1)}\right] v=\left(\begin{array}{c}
(0)_{3 \times 1} \\
i[-i]\left(\begin{array}{c}
\left(f_{1}^{\prime} H^{\prime}\right) \xi^{\prime} \\
0
\end{array}\right)
\end{array}\right),}
\end{aligned}
$$

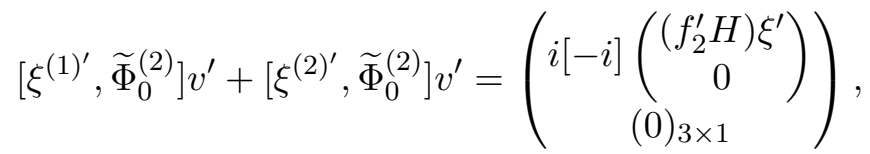

which immediately tells us about the non-zero linear combination, writing in general form under the condition $f_{1}^{\prime} \sim f_{2}^{\prime}$, after triplet-triplet splitting, the subtraction $\left(f_{1}^{\prime} H^{\prime}-f_{2}^{\prime} H\right) \xi^{\prime}$ gives immediately the Heisenberg scalar from $\mathrm{SU}(3), \xi^{\prime} H^{\prime \prime}$, as discussed in article 4.2.1. On the other side if $f_{2}^{\prime}>f_{1}^{\prime}$ and $H^{\prime}>H\left(H=H^{\prime}-H \neq 0\right)$ but further with $f_{1}^{\prime} H^{\prime} \sim f_{2}^{\prime} H$ vanishes, NGB $\xi^{\prime}$ decouples from the system. Clearly, PNB Higgs and other exotic scalars must be obtained from the other non-zero linear combination as below,

$$
\left[\xi^{(j)^{\prime}}, \widetilde{\Phi}_{0}^{(i)}\right] v\left[\left(v^{\prime}\right)\right]+\left[\xi^{(j)^{\prime}}, \widetilde{\Phi}_{0}^{(i)}\right] v^{\prime}[(v)] \neq 0,
$$

for $i=j=1,2$ and $i \neq j=1,2$ with $i=1$ for $v$ and $i=2$ for $v^{\prime}$. The first provides directly 2 (two) PNB Higgses, if NGB $\xi_{0}$ obtains non-zero VEV, $\left\langle\xi_{0}\right\rangle \neq 0$, as shown 
below,

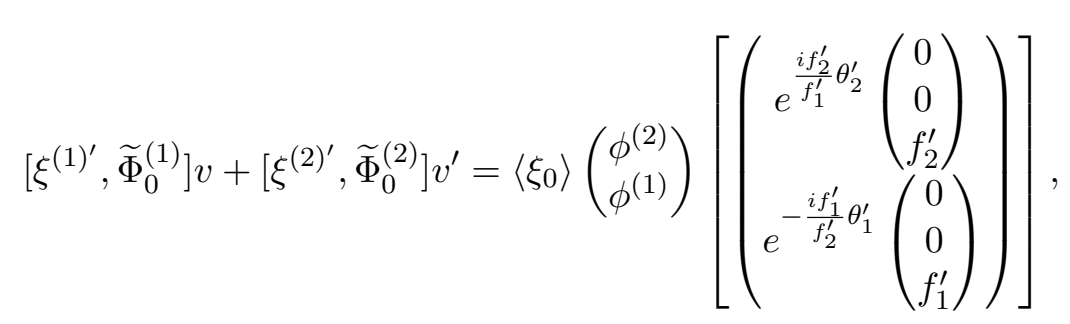

which splits directly into 2 (two) Little Higgs-like scalars which give two PNB Higgses or two degenerate Scherk-Schwarz scalars later on. From Eq. (115) one defines accordingly the second which is the dual of PNB Higgs as,

$$
\phi_{0 p}^{(1)^{\prime \prime}}\left[\phi_{0 p}^{(2)^{\prime \prime}}\right]=e^{-\frac{i f_{1}^{\prime}}{f_{2}^{\prime}}\left[\frac{i f_{2}^{\prime}}{f_{1}^{\prime}}\right] \theta_{1}^{\prime}\left[\theta_{2}^{\prime}\right]}\left(\begin{array}{c}
0 \\
0 \\
f_{1}^{\prime}
\end{array}\right)\left[\left(\begin{array}{c}
0 \\
0 \\
f_{2}^{\prime}
\end{array}\right)\right] .
$$

On the contrary the second gives clearly different exotic scalars, making use Eq. (109), Eq. (110) as shown below,

$$
\left[\xi^{(1)^{\prime}}, \widetilde{\Phi}_{0}^{(2)}\right] v^{\prime}+\left[\xi^{(2)^{\prime}}, \widetilde{\Phi}_{0}^{(1)}\right] v=\left(\begin{array}{c}
\left(\begin{array}{c}
i[-i]\left(f_{2}^{\prime} \xi^{\prime} H\right)_{2 \times 1} \\
0
\end{array}\right)-\left\langle\xi_{0}\right\rangle \phi^{(2)}\left[\phi_{0 p}^{(2)^{\prime \prime}}\right] \\
\left(\begin{array}{c}
i[-i]\left(f_{1}^{\prime} \xi^{\prime} H^{\prime}\right)_{2 \times 1} \\
0
\end{array}\right)-\left\langle\xi_{0}\right\rangle \phi^{(1)}\left[\phi_{0 p}^{(1)^{\prime \prime}}\right]
\end{array}\right)
$$

which can be further simplified by defining, applying first $f_{1}^{\prime} \sim f_{2}^{\prime} \sim\left\langle\xi_{0}\right\rangle$, as follows,

$$
\phi_{0}^{(1)^{\prime \prime}}\left[\phi_{0}^{(2)^{\prime \prime}}\right]=i[-i][i[-i]]\left(\begin{array}{c}
\left(\xi^{\prime} H^{\prime}[H]\right)_{2 \times 1} \\
0
\end{array}\right),
$$

where $\phi_{0}^{(1)^{\prime \prime}}\left[\phi_{0}^{(2)^{\prime \prime}}\right]$ contains the component of $H_{0}^{\prime \prime}$ which will be clear very soon.

Substituting Eq. (118) into Eq. (117) one obtains the simplified form having applied $f_{1}^{\prime} \sim f_{2}^{\prime} \sim\left\langle\xi^{\prime}\right\rangle$, as

$$
\left[\xi^{(1)^{\prime}}, \widetilde{\Phi}_{0}^{(2)}\right] v^{\prime}+\left[\xi^{(2)^{\prime}}, \widetilde{\Phi}_{0}^{(1)}\right] v=\left\langle\xi_{0}\right\rangle\left(\begin{array}{l}
\phi_{0}^{(2)^{\prime \prime}}-\phi^{(2)}\left[\phi_{0 p}^{(2)^{\prime \prime}}\right] \\
\phi_{0}^{(1)^{\prime \prime}}-\phi^{(1)}\left[\phi_{0 p}^{(1)^{\prime \prime}}\right]
\end{array}\right),
$$

which also shows the linear combination of $\phi_{0}^{(i)^{\prime \prime}}$ with both Little Higgs-like scalars $\phi^{(i)}$ and degenerate Scherk-Schwarz scalars $\phi_{0 p}^{(i)^{\prime \prime}}, i=1,2$. This property, under triplet-triplet splitting, indicates the potential for the emerging of new exotic scalars which will be discussed in the next section.

Before concluding this section we recall the swallowing-digesting mode which characterizes the sextets subjected to triplet-triplet splitting as below

Eq. (115) shows clearly the vanishing of $\left(2 \xi^{\prime}\right)$ after triplet-triplet splitting takes place where, eventhough $2 \times\left(2 \xi^{\prime}\right)$ NGBs exist in $\left(\xi^{(1)^{\prime}}\right)$ and $\left(\xi^{(2)^{\prime}}\right)$ at the lefthand side but, no NGB shows up at the righthand side. No NGB-mass form shows up 
indicating both the existence of swallowing mode and zero additional mass in the formation of $\phi_{0 p}^{(i)^{\prime \prime}}, i=1,2$.

On the contrary Eq. (118) shows vividly the digesting mode in the formation of $\phi_{0}^{(i)^{\prime \prime}}, i=1,2$ where under unitary property $\xi^{\prime \dagger} \xi^{\prime}=1$ one can multiply $\xi^{\prime} H^{\prime}[H]$ with factor $1\left(=\frac{\xi^{\prime \dagger}}{\left(\xi^{\prime}\right)^{-1}}\right)$, to obtain the following equality $\xi^{\prime} H^{\prime}[H]=\frac{\xi^{\prime \dagger} \xi^{\prime}}{\left(\xi^{\prime}\right)^{-1}} H^{\prime}[H]=$ $\left(\xi^{\prime \dagger} \xi^{\prime}\right) \xi^{\prime} H^{\prime}[H]$ which shows clearly the NGB-mass form indicating the transferring of extra mass together with 2 (two) degrees of freedom confirming $\phi_{0}^{(i)^{\prime \prime}} \neq \phi_{0 p}^{(i)^{\prime \prime}}, i=1,2$ and $m_{H_{0}^{\prime \prime}}>m_{H_{0}^{\prime \prime}}$.

\section{The conservation and masses of unified Higgs-NGB in the formation of Scherk-Schwarz and the other exotic scalars}

\subsection{The 3-component scalar from conservation of unification of Higgs-NGB under unitarily-transformed SU(6) symmetry break- ing}

After triplet-triplet splitting SU(3)-level allows a linear combination of triplets, making use Eq. (118) in this case, and further subtracting the lower triplet with the upper triplet one finds a field redefinition, similar to orbifold-based field redefinition in [3], as follows,

$$
\phi_{0}^{\prime \prime}=\left(\phi_{0}^{(1)^{\prime \prime}}-\phi_{0}^{(2)^{\prime \prime}}\right)-\phi^{(1)}\left[\phi_{0 p}^{(1)^{\prime \prime}}\right]+\phi^{(2)}\left[\phi_{0 p}^{(2)^{\prime \prime}}\right],
$$

allowing the Little Higgs-like scalar and its gauge-like dual to obtain non-zero VEVs $\left\langle\phi^{(1)}\right\rangle\left[\left\langle\phi_{0 p}^{(1)^{\prime \prime}}\right\rangle\right]$ and $\left\langle\phi^{(2)}\right\rangle\left[\left\langle\phi_{0 p}^{(2)^{\prime \prime}}\right\rangle\right]$, one obtains

$$
\phi_{0}^{\prime \prime}=\left(\phi_{0}^{(1)^{\prime \prime}}-\phi_{0}^{(2)^{\prime \prime}}\right)-\left\langle\phi^{(1)}\right\rangle\left[\left\langle\phi_{0 p}^{(1)^{\prime \prime}}\right\rangle\right]+\left\langle\phi^{(2)}\right\rangle\left[\left\langle\phi_{0 p}^{(2)^{\prime \prime}}\right\rangle\right]
$$

defining first $\phi_{0}^{(12)^{\prime \prime}}=\left(\phi_{0}^{(1)^{\prime \prime}}-\phi_{0}^{(2)^{\prime \prime}}\right)$ then from which, neglecting the constants and utilising Eq. (118), one also obtains the following expression,

$$
\phi_{0}^{(12)^{\prime \prime}}=i[-i]\left(\begin{array}{c}
\left(\xi^{\prime}\left(H^{\prime}-H\right)\right)_{2 \times 1} \\
0
\end{array}\right)=i[-i]\left(\begin{array}{c}
\left(\xi^{\prime}\left(H^{\prime \prime}\right)\right)_{2 \times 1} \\
0
\end{array}\right),
$$

where doublet $H^{\prime \prime}$ absorbs 2 (two) NGBs, $\xi^{\prime} H^{\prime \prime}=\left(\xi^{\prime} H_{1}^{\prime \prime} \quad \xi^{\prime} H_{2}^{\prime \prime}\right)^{T}$, and $\phi_{0}^{\prime \prime}$ obtains extra mass following the above digesting mode. Here, one retrieves again the definition of Heisenberg scalar in subsection 4.2.1, $H_{H}^{\prime \prime}=\xi^{\prime} H^{\prime \prime}$ and rewrites Eq. (122) accordingly as,

$$
\phi_{0}^{(12)^{\prime \prime}}=i[-i]\left(\begin{array}{c}
\left(H_{H}^{\prime \prime}\right)_{2 \times 1} \\
0
\end{array}\right)
$$

which demonstrates the restoration of unified Higgs-NGB fields as the exotic ScherkSchwarz scalar type-1, in other words, the unification of Higgs-NGB is conserved after SU(6) symmetry breaking under unitary transformation. 
In case $\phi^{(i)}\left[\phi_{0 p}^{(i)^{\prime \prime}}\right], i=1,2$ does not obtain non-zero VEV in Eq. (121) and still be in massless state the Higgs-NGB unification is still conserved but a new phenomenon shows up i.e. the emerging of exotic multi-component scalars which is discussed below. From Eq. (120) one expands $\phi^{(i)}, i=1,2$ to the lowest order, following Eq. (26) and neglecting $\left(\begin{array}{ll}0 & 0\end{array} f^{\prime}\right)^{T}, \Delta f^{\prime}=\left(f_{2}^{\prime}-f_{1}^{\prime}\right) \sim 0$ to obtain finally the result as below,

$$
\phi_{0}^{\prime \prime}=i[-i]\left(\begin{array}{c}
H_{H}^{\prime \prime} \\
0
\end{array}\right)-\frac{i f_{2}^{\prime}}{f^{\prime}}\left(\begin{array}{c}
H^{\prime} \\
0
\end{array}\right)-\frac{i f_{1}^{\prime}}{f^{\prime}}\left(\begin{array}{c}
H \\
0
\end{array}\right),
$$

or, in short forms, as

$$
\phi_{0}^{\prime \prime}=\left(\begin{array}{c}
H_{3 h}^{\prime \prime(1)}\left[H_{3 h}^{\prime \prime(2)}\right] \\
0
\end{array}\right),
$$

where the following definitions have been adopted, after simplifying further by taking $f_{1}^{\prime} \sim f_{2}^{\prime} \sim f^{\prime}$

$$
H_{3 h}^{\prime \prime(1)}\left[H_{3 h}^{\prime \prime(2)}\right]=i[-i]\left\{H_{H}^{\prime \prime}-\left(H^{\prime}+H\right)\left[+\left(H^{\prime}+H\right)\right]\right\},
$$

here, $H_{H}^{\prime \prime}$ a gauge-like Scherk-Schwarz scalar type-1 (Heisenberg scalar), $H^{\prime}[H]$ PNB Higgs and $H_{3 h}^{\prime \prime(i)}, i=1,2$ is called as 3-scalar Higgs with 1 (one) degree of freedom.

Another variant scalar is also given by Eq. (120) making use $\phi_{0 p}^{(i)^{\prime \prime}}$ instead of $\phi^{(i)}, i=1,2$ from which one finds $\phi_{0 p}^{\prime \prime}$, further denoting $\phi_{0}^{\prime \prime}$ by $\phi_{0 p}^{\prime \prime}$, also approximating $\theta_{i}^{\prime}, i=1,2$ and substituting with $\theta_{1}^{\prime} \sim \frac{1}{f^{\prime}}\left(\begin{array}{ccc}0 & 0 \\ 0 & 0 \\ \left(H^{\dagger}+\epsilon_{0} \xi^{\dagger}\right.\end{array}\right)\left(\begin{array}{cc}\left(H^{\prime}+\epsilon_{0} \xi^{\prime}\right) \\ 0\end{array}\right), \theta_{2}^{\prime} \sim$ $\frac{1}{f^{\prime}}\left(\begin{array}{ccc}0 & 0 & \left(H+\epsilon_{0} \xi^{\prime}\right.\end{array}\right), \epsilon_{0} \rightarrow 0$, finally neglecting $\left(\begin{array}{lll}0 & 0 & \Delta f^{\prime}\end{array}\right)^{T}, \Delta f^{\prime}=\left(f_{2}^{\prime}-f_{1}^{\prime}\right) \sim 0$, and obtains the final result as follows,

$$
\phi_{0 p}^{\prime \prime}=i[-i]\left(\begin{array}{c}
H_{H}^{\prime \prime} \\
0
\end{array}\right)+\frac{i f_{1}^{\prime 2}}{f_{2}^{\prime} f^{\prime}}\left(\begin{array}{c}
\left(H^{\prime}+\epsilon_{0} \xi^{\prime}\right) \\
0
\end{array}\right)+\frac{i f_{2}^{\prime 2}}{f_{1}^{\prime} f^{\prime}}\left(\begin{array}{c}
\left(H+\epsilon_{0} \xi^{\prime}\right) \\
0
\end{array}\right) .
$$

Recalling Eq. (79) the $\mathrm{SU}(6) H_{0 p}^{\prime \prime(+)}$ can be rewritten in its components, $H_{0 p}^{\prime \prime(+)}=$ $H_{0 p(1)}^{\prime \prime(+)}+H_{0 p(2)}^{\prime \prime(+)}$, where the following definitions have been used,

$$
H_{0 p(1)}^{\prime \prime(+)}=\left(H^{\prime}+\epsilon_{0} \xi^{\prime}\right), \quad H_{0 p(2)}^{\prime \prime(+)}=\left(H+\epsilon_{0} \xi^{\prime}\right) .
$$

Under triplet-triplet splitting the two components also split due to each resides in different triplet as demonstrated clearly by Eq. (119) where triplets $\left(H_{0}\right)^{T}$ and $\left(\begin{array}{ll}H^{\prime} & 0\end{array}\right)^{T}$ are completely separated. Therefore Eq. (127) represents a 3-component scalar which can be rewritten as,

$$
\phi_{0 p}^{\prime \prime}=\left(\begin{array}{c}
H_{3 s}^{\prime \prime(1)}\left[H_{3 s}^{\prime \prime(2)}\right] \\
0
\end{array}\right),
$$

where further simplification has been applied utilising $f_{1}^{\prime} \sim f_{2}^{\prime} \sim f^{\prime}$ and the definition below has been established,

$$
H_{3 s}^{\prime \prime(1)}\left[H_{3 s}^{\prime \prime(2)}\right]=i[-i]\left\{H_{H}^{\prime \prime} \pm\left(H^{\prime}+\epsilon_{0} \xi^{\prime}\right) \pm\left(H+\epsilon_{0} \xi^{\prime}\right)\right\},
$$


with (-) sign for $[-i]$. It is clear that one can extract 2 (two) exotic scalars either with 3 (three) or 1 (one) degrees of freedom i.e. a gauge-like and a Higgs-like 3component scalar denoted by $H_{3 s}^{\prime \prime(1)}$ and $H_{3 s}^{\prime \prime(2)}$ respectively as shown below,

$$
\begin{aligned}
& H_{3 s}^{\prime \prime(1)}=i\left(H_{H}^{\prime \prime}+H_{0 p(1)}^{\prime \prime(+)}+H_{0 p(2)}^{\prime \prime(+)}\right), \\
& H_{3 s}^{\prime \prime(2)}=-i\left(H_{H}^{\prime \prime}-H_{0 p(1)}^{\prime \prime(+)}-H_{0 p(2)}^{\prime \prime(+)}\right),
\end{aligned}
$$

from Eq. (131) one predicts a gauge-like 3-component scalar due to equally-distributed 2 (two) openly-constrained degrees of freedom and 1 (one) free degree of freedom which belong to each component scalar $H_{H}^{\prime \prime}, H_{0 p(1)}^{\prime \prime(+)}$ and $H_{0 p(2)}^{\prime \prime(+)}$, reflected by each term's $(+)$ sign of each component.

On the contrary Eq. (132) predicts a Higgs-like 3-component scalar due to different arrangement of degrees of freedom i.e 2 (two) closely-constrained and 1 (one) openly-constrained degrees of freedom which belong to each $H_{0 p(i)}^{\prime \prime(+)}, i=1,2$ with each term's minus sign while 2 (two) openly-constrained and 1 (one) free degrees of freedom belong to $H_{H}^{\prime \prime}$, leaving finally only 1 (one) free degree of freedom for the 3-component scalar. Both $H_{3 s}^{\prime \prime(1)}$ and $H_{3 s}^{\prime \prime(2)}$ are drawn nicely in schematic drawings given in Appendix C.

Following Eq. (78) and Eq. (79) one allows $\epsilon \rightarrow \sim 1$ so that $H_{0 p(i)}^{\prime \prime(+)} \rightarrow H_{0(i)}^{\prime \prime(+)}$, $\left\langle\phi_{0 p}^{(i)^{\prime \prime}}\right\rangle \rightarrow\left\langle\phi_{00}^{(i)^{\prime \prime}}\right\rangle, \phi_{0 p}^{\prime \prime} \rightarrow \phi_{00}^{\prime \prime} H_{3 s}^{\prime \prime(i)} \rightarrow H_{30}^{\prime \prime(i)}, \theta_{i}^{\prime} \rightarrow \theta_{0 i}^{\prime}$ and Eq. (128), Eq. (130) to Eq. (132) become accordingly as,

$$
\begin{aligned}
H_{0(1)}^{\prime \prime(+)} & =\left(H^{\prime}+\xi^{\prime}\right), \quad H_{0(2)}^{\prime \prime(+)}=\left(H+\xi^{\prime}\right), \\
H_{30}^{\prime \prime(1)}\left[H_{30}^{\prime \prime(2)}\right] & =i[-i]\left\{H_{H}^{\prime \prime} \pm H_{0(1)}^{\prime \prime(+)} \pm H_{0(2)}^{\prime \prime(+)}\right\},
\end{aligned}
$$

which provides both gaugle-like and Higgs-like 3-component scalar where each component $H_{H}^{\prime \prime}, H_{0(1)}^{\prime \prime(+)}, H_{0(2)}^{\prime \prime(+)}$ has the equal mass. Here, one finds another variant 3 component scalar with respect to $H_{3 s}^{\prime \prime(i)}, i=1,2$.

To conclude, the conservation of unification of Higgs-NGB under unitary transformation after $\mathrm{SU}(6)$ symmetry breaking provides and guarantees stable exotic scalars in $\mathrm{SU}(3)$ consisting of 2 (two) types i.e. Higgs-like and gauge-like 3-component scalars. Next, it is time to analyze the masses of exotic scalars and its sources of production in the symmetry breakings which will be discussed shortly in the next section.

\subsection{Masses of Scherk-Schwarz scalars from triplet-triplet splitting potential}

\subsubsection{SU(6)-origin type-2 Scherk-Schwarz scalar from the generalized Coleman-Weinberg potential}

With the aid of Eq. (23), Eq. (46) the triplet-triplet splitting potential is obtained directly from Eq. (80) and Eq. (81) by substituting $\widetilde{\Phi}^{(i)} \rightarrow \widetilde{\Phi}^{(i)^{\prime}}$ and $\widetilde{\Phi}_{\mathrm{NG}}^{(j)} \rightarrow \widetilde{\Phi}_{\mathrm{NG}}^{(j)^{\prime}}, i, j=$ 
1,2 , after which the trace vanishes with the potential, in general, is non-zero. This indicates the emerging of a massive particle which can be either a PNB Higgs or a type-2 Scherk-Schwarz scalar. Referring back to Eq. (22) and Eq. (46) potential terms of generalized Coleman-Weinberg potential consisting of $\widetilde{\Phi}^{(i)^{\prime} \dagger} \Phi_{\mathrm{NG}}^{(j)^{\prime}}\left[\Phi_{\mathrm{NG}}^{(j)^{\prime} \dagger} \widetilde{\Phi}^{(i)^{\prime}}\right]$ where $\widetilde{\Phi}^{(i)^{\prime}}=\widetilde{\Phi}_{0}^{(i)^{\prime}} v\left[v^{\prime}\right]$ or $\widetilde{\Phi}_{0}^{(i)^{\prime}} v^{\prime}[v]$ brings to the important property of the above equation. It has been predicted for $i=j=1,2$ that PNB Higgs or its degenerate Scherk-Schwarz scalar (type-2B) will show up as indicated by Eq. (115) while for $i \neq j=1,2$ exotic scalars including a heavier Scherk-Schwarz scalar (type-1) in Eq. (123) and 3-component Higgs and scalars in Eq. (126) and Eq. (130) also Eq. (133) will emerge accordingly.

Let's start with rewriting the potential as,

$$
\begin{aligned}
V_{\mathrm{NP} g}^{(6)} & =V_{\mathrm{NP} g(i=j)}^{(6)}+V_{\mathrm{NP} g(i \neq j)}^{(6)}, \\
V_{\mathrm{NP} g(i=j)}^{(6)} & =\lambda_{y \mathrm{NP}}^{(6)}\left(\widetilde{\Phi}^{(i)^{\prime} \dagger} \Phi_{\mathrm{NG}}^{()^{\prime}} \Phi_{\mathrm{NG}}^{(i)^{\prime} \dagger} \widetilde{\Phi}^{(i)^{\prime}}+\Phi_{\mathrm{NG}}^{(i)^{\prime} \dagger} \widetilde{\Phi}^{(i)^{\prime}} \widetilde{\Phi}^{(i)^{\prime} \dagger} \Phi_{\mathrm{NG}}^{(i)^{\prime}}\right), \quad i, j=1,2, \\
V_{\mathrm{NP} g(i \neq j)}^{(6)} & =\lambda_{y \mathrm{NP}}^{(6)}\left(\widetilde{\Phi}^{(i)^{\prime} \dagger} \Phi_{\mathrm{NG}}^{(j)^{\prime}} \Phi_{\mathrm{NG}}^{(j)^{\prime} \dagger} \widetilde{\Phi}^{(i)^{\prime}}+\Phi_{\mathrm{NG}}^{(j)^{\prime} \dagger} \widetilde{\Phi}^{(i)^{\prime}} \widetilde{\Phi}^{(i)^{\prime} \dagger} \Phi_{\mathrm{NG}}^{(j)^{\prime}}\right), \quad i, j=1,2 .
\end{aligned}
$$

Substituting Eq. (23) and (46) into Eq. (135), separating and neglecting the mixed-up terms such as $\phi_{0}^{(i) \dagger} \phi_{\mathrm{NG}}^{(i)} \phi_{0}^{(i) \dagger} \phi^{(i)}, \quad \phi^{(i) \dagger} \phi_{0}^{(i)} \phi_{\mathrm{NG}}^{(i) \dagger} \phi_{0}^{(i)}, \quad \phi_{\mathrm{NG}}^{(i) \dagger} \phi_{0}^{(i)} \phi^{(i) \dagger} \phi_{0}^{(i)}$ and $\phi_{0}^{(i) \dagger} \phi^{(i)} \phi_{0}^{(i) \dagger} \phi_{\mathrm{NG}}^{(i)}$ since it produces entities $\left(\theta_{i}^{\prime}+\xi_{\mathrm{ng}}^{\prime}\right),\left(\theta_{i}^{\prime \dagger}+\xi_{\mathrm{ng}}^{\prime}\right), i=1,2$ and its linear combinations which facilitate double vacua with double shifts as shown by Eq. (47) which are not twistedly-broken shift breaking terms in Eq. (95), and keep shift symmetry intact, one can finally simplify Eq. (135) to become, replacing $\lambda_{y \mathrm{NP}}^{(6)}$ with $\lambda^{(3)}$, and to facilitate triplet-triplet splitting as

$$
\begin{aligned}
V_{\mathrm{NP} g(i=j)}^{(6)}=\lambda^{(3)}\{ & \left(\phi_{0}^{(i) \dagger} \phi_{\mathrm{NG}}^{(i)} \phi_{\mathrm{NG}}^{(i) \dagger} \phi_{0}^{(i)}+\phi_{\mathrm{NG}}^{(i) \dagger} \phi_{0}^{(i)} \phi_{0}^{(i) \dagger} \phi_{\mathrm{NG}}^{(i)}\right) \\
& \left.+\left(\phi^{(i) \dagger} \phi_{0}^{(i)} \phi_{0}^{(i) \dagger} \phi^{(i)}+\phi_{0}^{(i) \dagger} \phi^{(i)} \phi^{(i) \dagger} \phi_{0}^{(i)}\right)\right\},
\end{aligned}
$$

where $i=j=1,2$. Eq. (137) shows resemblance with respect to the lefthand sides of Eq. (100) to Eq. (102), if one sets and associates the following $\xi^{(i)^{\prime}} \rightarrow$ $\xi_{n g}^{\prime} \rightarrow \phi_{\mathrm{NG}}^{(i)}, i=1,2$ while for $\widetilde{\Phi}^{(i)} \rightarrow \phi_{0}^{(i)}, i=1,2$ to obtain for the first term of Eq. (137). Analogously, for the second term the association takes on the form $\xi^{(i)^{\prime}} \rightarrow\left\langle\xi_{0}^{\prime}\right\rangle \rightarrow \phi_{0}^{(i)}, i=1,2$ while for $\widetilde{\Phi}^{(i)} \rightarrow \phi^{(i)}, i=1,2$. This association indicates clearly the twistedly-broken shift breaking property in Eq. (137), and further the taking place of Higgs-NGB unification. The first term provides immediately the constant and the second term the mass term as follows,

$$
V_{\mathrm{NP} g(i=j)}^{(6)}=2\left(f_{1}^{\prime 4}+f_{2}^{\prime 4}\right)+\frac{2 f_{1}^{\prime 2} f_{2}^{\prime 2}}{f^{\prime 2}} H^{\prime \prime \dagger} H^{\prime \prime}
$$

where one finds convincingly that the mass term $H^{\prime \prime \dagger} H^{\prime \prime}$ in Eq. (138) is yielded without any direct influence from NGB as clearly shown by no-NGB term in the second term. Nevertheless, based on Eq. (115) the two NGBs must have decoupled from PNB Higgs $H_{i}^{\prime \prime}, i=1,2$ or swallowed (joined loosely and masslessly) to PNB 
Higgs in such a way it does not add (or affect) the mass of PNB Higgs but contributes extra degrees of freedom. The later is the 2B-type state of Scherk-Schwarz scalar socalled swallowing mode where the vanished NGBs have also been shown by Eq. (116). One names accordingly either as $H_{i}^{\prime \prime}, i=1,2$ the PNB Higgses or $H_{0 p}^{(i)^{\prime \prime}}, i=1,2$ the swallowing-state Scherk-Schwarz scalars (type-2B), with the mass-squared as follows,

$$
m_{H_{i}^{\prime \prime}}^{2}=m_{H_{0 p}^{(i)^{\prime \prime}}}^{2}=\frac{g^{\prime 4}}{16 \pi^{2}} \frac{\lambda^{(3)}}{f^{\prime 2}}\left(2 f_{1}^{\prime 2} f_{2}^{\prime 2}\right) \log \left(\frac{\Lambda_{(3)}^{2}}{\mu_{H_{i}^{\prime \prime}\left[H_{0 p}^{(i)^{\prime \prime}}\right]}^{2}}\right), i=1,2,
$$

where $\mathcal{O}\left(\mu_{H_{i}^{\prime \prime}}\right) \sim \mathcal{O}\left(\mu_{H_{0 p}^{(i)^{\prime \prime}}}\right) \sim \mathcal{O}(100 \mathrm{GeV}), g^{\prime}$ the $\mathrm{SU}(3)$ coupling constant, and $\Lambda_{(3)}$ cut-off scale.

On the contrary, one can also substitute Eq. (23) and (46) into Eq. (136) and find the mixed-up terms to be neglected, such as $\phi_{0}^{(i) \dagger} \phi_{0}^{(j)} \phi_{\mathrm{NG}}^{(j) \dagger} \phi^{(i)}, \phi^{(i) \dagger} \phi_{\mathrm{NG}}^{(j)} \phi_{0}^{(j) \dagger} \phi_{0}^{(i)}$, $\phi_{0}^{(j) \dagger} \phi_{0}^{(i)} \phi^{(i) \dagger} \phi_{\mathrm{NG}}^{(j)}$ and $\phi_{\mathrm{NG}}^{(j) \dagger} \phi^{(i)} \phi_{0}^{(i) \dagger} \phi_{0}^{(j)}$, based on the same reason as above, so that Eq. (136) is simplified, replacing $\lambda_{y \mathrm{NP}}^{(6)}$ with $\lambda^{(3)}$, as below,

$$
\begin{aligned}
V_{\mathrm{NP} g(i \neq j)}^{(6)}=\lambda^{(3)}\{ & \left(\phi_{0}^{(i) \dagger} \phi_{0}^{(j)} \phi_{0}^{(j) \dagger} \phi_{0}^{(i)}+\phi_{0}^{(j) \dagger} \phi_{0}^{(i)} \phi_{0}^{(i) \dagger} \phi_{0}^{(j)}\right) \\
& \left.+\left(\phi^{(i) \dagger} \phi_{\mathrm{NG}}^{(j)} \phi_{\mathrm{NG}}^{(j) \dagger} \phi^{(i)}+\phi_{\mathrm{NG}}^{(j) \dagger} \phi^{(i)} \phi^{(i) \dagger} \phi_{\mathrm{NG}}^{(j)}\right)\right\},
\end{aligned}
$$

where $i \neq j=1,2$. The twistedly-broken shift breaking property can be seen clearly from the second term of Eq. (140) in accordance to Eq. (95). The first term is just a constant while the second the anticipated mass term as shown below,

$$
V_{\mathrm{NP} g(i \neq j)}^{(6)}=4\left(f_{1}^{\prime 2} f_{2}^{\prime 2}\right)+\frac{f_{1}^{\prime 4}+f_{2}^{\prime 4}}{f^{\prime 2}} H^{\prime \prime \dagger} H^{\prime \prime}
$$

where one finds without any doubt that the mass term $H^{\prime \prime \dagger} H^{\prime \prime}$ in Eq. (141) is obtained from the previously-cited combination of Higgs and NGBs as shown by Eq. (102) followed by Eq. (104) where additional mass and degrees of freedom take place simultaneously. The second term of Eq. (140), which is actually the previouslydefined Scherk-Schwarz scalar type-2A in the so-called digesting mode where NGBs have coupled strongly and added some masses to Higgs PNB, to give $H_{0}^{\prime \prime}$, with the following mass-squared,

$$
m_{H_{0}^{\prime \prime}}^{2}=\frac{g^{\prime 4}}{16 \pi^{2}} \frac{\lambda^{(3)}}{f^{\prime 2}}\left(f_{1}^{\prime 4}+f_{2}^{\prime 4}\right) \log \left(\frac{\Lambda_{(3)}^{2}}{\mu_{H_{0}^{\prime \prime}}^{2}}\right),
$$

where $\mathcal{O}\left(\mu_{H_{0}^{\prime \prime}}\right) \sim \mathcal{O}(100 \mathrm{Ge} V)$.

Both PNB Higgs and Scherk-Schwarz scalar type-2A and -2B become the basic constituents of exotic scalars where the formation of Scherk-Schwarz scalar from a Higgs and 2 (two) NGBs including the transfer of degree of freedom and mass generation requires a new mechanism which has been discussed in the previous sections. 
Before closing the subsection, one is reminded that Eq. (142) does show a standalone free Scherk-Schwarz scalar (type-2A) and can be regarded as a gauge-like single scalar which also, quite possibly, can serve as a candidate of relic dark matter $[12,20]$. Nevertheless one also faces the hidden Scherk-Schwarz scalar (type-2B) which can basically be found as a bonded scalar, jointed with either PNB Higgses or itselves to form exotic multi-component Higgses and scalars. This is to be discussed in the next paper in the more detail way. Since conservation of unification of Higgs-NGB takes place after triplet-triplet splitting which triggers the emerging of $\mathrm{SU}(6)$-origin Heisenberg scalar as discussed in section 6.1. The mass of this scalar must have been represented by Eq. (141) as well so that one also has $m_{H_{H}^{\prime \prime}}=m_{H_{0}^{\prime \prime}}$. Therefore $H_{H}^{\prime \prime}$ together with $H_{i}^{\prime \prime}\left[H_{0 p}^{\prime \prime}(i)\right], i=1,2$ become the components of exotic scalars.

It is clear that total potential $V_{N P g}^{(6)}$ expressed in Eq. (134) gives an exotic 3scalar Higgs and exotic 3-component Higgs-like (and gauge-like) scalar as depicted in Eq. (126) [and Eq. (130)], also Eq. (133) with the masses as shown below,

$$
\begin{aligned}
& m_{H_{3 h}^{\prime \prime(i)}}^{2}=m_{H_{3 s}^{\prime \prime(i)}}^{2}=\frac{g^{\prime 4}}{16 \pi^{2}} \frac{\lambda^{(3)}}{f^{\prime 2}}\left\{\left(2 f_{1}^{\prime 2} f_{2}^{\prime 2}\right)+\left(f_{1}^{\prime 4}+f_{2}^{\prime 4}\right)\right\} \log \left(\frac{\Lambda_{(3)}^{2}}{\mu_{H_{3 h(s)}^{\prime \prime(i)}}^{2}}\right), i=1,2, \\
& m_{H_{30}^{\prime \prime(i)}}^{2}=3 m_{H_{0}^{\prime \prime}}^{2}
\end{aligned}
$$

where $\mathcal{O}\left(\mu_{H_{3 h(s)}^{\prime \prime(i)}}\right) \sim \mathcal{O}(100 \mathrm{GeV})$, mass coupling $H_{0 p(i)}^{\prime \prime(+)}, i=1,2$ are set at $\mu_{H_{0 p(1)}^{\prime \prime(+)}}^{2}=$ $\mu_{H_{0 p(2)}^{\prime \prime(+)}}^{2}=\frac{\lambda^{(3)}}{f^{\prime 2}}\left(f_{1}^{\prime 2} f_{2}^{\prime 2}\right)$. For clarity one can symbolize a 3-scalar Higgs based on Eq. (126), as $H_{3 h}^{\prime \prime(i)} \equiv\left(H_{i}^{\prime \prime}-H_{H}^{\prime \prime}-H_{i}^{\prime \prime}\right), i=1,2$ and a Higgs-like 3-component scalar, based on Eq. (132) as $\left.H_{3 s}^{\prime \prime(2)} \equiv\left(H_{H}^{\prime \prime}-H_{0 p(2)}^{\prime \prime(+)}\right)=H_{0 p(1)}^{\prime \prime(+)}\right)$, with a gauge-like 3-component scalar from Eq. (131) as $H_{3 s}^{\prime \prime(1)} \equiv\left(H_{0 p(1)}^{\prime \prime(+)}-H_{H}^{\prime \prime}-H_{0 p(2)}^{\prime \prime(+)}\right)$ with schematic drawings as shown in Appendix C.

\subsubsection{SU(3)-origin type-1 Scherk-Schwarz scalar from Generalized Cole- man Weinberg potential}

In the global $\mathrm{SU}(3) \times \mathrm{SU}(3) \times \mathrm{U}(1)$ symmetry breaking the broken symmetry is basically $\mathrm{SU}(3)_{H_{H}^{\prime \prime}} \times \mathrm{U}(1)_{H_{0}^{\prime \prime}}$ which produces 2 scalar-pairs and one Scherk-Schwarz scalar from $\mathrm{SU}(3)_{H_{H}^{\prime \prime}}$ and another one from $\mathrm{U}(1)_{H_{0}^{\prime \prime}}$. The first is grouped into type-1 and the second into type-2A following the grouping previously given in subsection (4.2.1) and (4.2.2). One selects and writes the wavefunction in $\mathrm{SU}(3) \times \mathrm{U}(1)$, without loss of generality, as follows,

$$
\phi_{N G}^{(j)}=v_{\mathrm{NG}}^{(j)}\left(e^{ \pm \frac{i f_{i}^{\prime}}{f_{j}^{\prime}} \frac{\xi}{f^{\prime}}}\right)\left(e^{ \pm \frac{i f_{i}^{\prime}}{f_{j}^{\prime}} \frac{\xi \lambda_{8}}{f^{\prime}}}\right), \quad i \neq j=1,2,
$$

where $v_{\mathrm{NG}}^{(j)}=\left(\begin{array}{lll}0 & 0 & f_{j}^{\prime}\end{array}\right)^{T}, \xi$ is NGB, $\lambda_{8}$ is the last generator of $\mathrm{SU}(3)$ and $(+)$ for

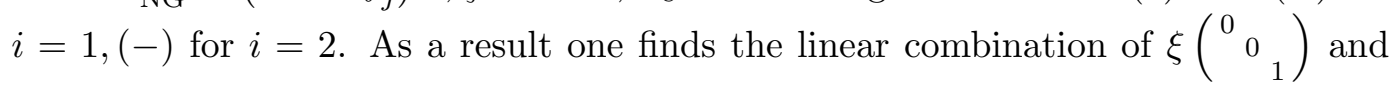




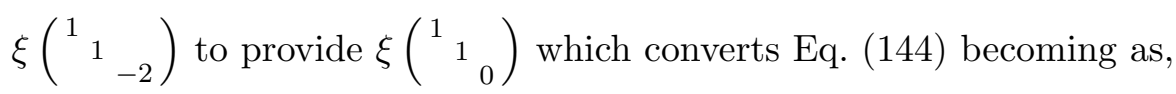

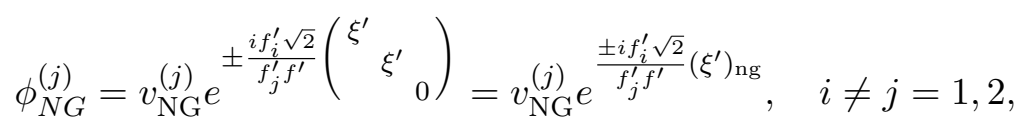

where factor $\frac{1}{\sqrt{2}}$ is absorbed into $\xi^{\prime}$ and $\left(\xi^{\prime}\right)_{\text {ng }}$ is restated below.

From Eq. (145) one can also write SimplestLittleHiggs-like scalar in short form as,

$$
\phi^{(1)}\left[\phi^{(2)}\right]=\phi_{0}^{(1)}\left[\phi_{0}^{(2)}\right] e^{\frac{i f_{2}^{\prime}}{f_{1}^{\prime} f^{\prime}}\left[\frac{-i f_{1}^{\prime}}{f_{2}^{\prime} f^{\prime}}\right](H)_{\mathrm{pnb}}^{\dagger}\left[(H)_{\mathrm{pnb}}\right]},
$$

where the following definitions have been used in Eq. (145) and Eq. (146) above,

$$
(H)_{\mathrm{pnb}}=\left(\begin{array}{ccc}
0 & 0 & \\
0 & 0 & H \\
H^{\prime \dagger} & 0
\end{array}\right) \text { and }\left(\xi^{\prime}\right)_{\mathrm{ng}}=\left(\begin{array}{ccc}
\xi^{\prime} & & \\
& \xi^{\prime} & \\
& & 0
\end{array}\right)
$$

in consistency with previous definitions in Eq. (29), (30) and Eq. (45).

The Lagrangian in near-brane which is basically a Coleman-Weinberg potential becomes as,

$$
\begin{aligned}
V & =V_{1}+V_{2}, \\
V_{1} & =\lambda^{(3)}\left\{\phi^{(1) \dagger} \phi_{\mathrm{NG}}^{(1)} \phi_{\mathrm{NG}}^{(1) \dagger} \phi^{(1)}+\phi_{\mathrm{NG}}^{(1) \dagger} \phi^{(1)} \phi^{(1) \dagger} \phi_{\mathrm{NG}}^{(1)}\right\}, \\
V_{2} & =\lambda^{(3)}\left\{\phi^{(2) \dagger} \phi_{\mathrm{NG}}^{(2)} \phi_{\mathrm{NG}}^{(2) \dagger} \phi^{(2)}+\phi_{\mathrm{NG}}^{(2) \dagger} \phi^{(2)} \phi^{(2) \dagger} \phi_{\mathrm{NG}}^{(2)}\right\},
\end{aligned}
$$

with $V_{1}$ and $V_{2}$ can be expressed in compact forms, making use Eq. (144) and Eq. (145), as

$$
V_{1}=\lambda^{(3)}\left[\phi_{0}^{(1) T} v_{\mathrm{NG}}^{(1)} e^{-\frac{i f_{2}^{\prime} \sqrt{2}}{f_{1}^{\prime} f^{\prime}}\left\{\left(\xi^{\prime}\right)_{\mathrm{ng}}+\frac{1}{\sqrt{2}}(H)_{\mathrm{pnb}}\right\}}\right]\left[v_{\mathrm{NG}}^{(1) T} \phi_{0}^{(1)} e^{\frac{i f_{2}^{\prime} \sqrt{2}}{f_{1}^{\prime} f^{\prime}}\left\{\left(\xi^{\prime}\right)_{\mathrm{ng}}+\frac{1}{\sqrt{2}}(H)_{\mathrm{pnb}}^{\dagger}\right\}}\right]+h c
$$

Analogously, one also finds the expression below,

$$
V_{2}=\lambda^{(3)}\left[\phi_{0}^{(2) T} v_{\mathrm{NG}}^{(2)} e^{\frac{i f_{2}^{\prime} \sqrt{2}}{f_{2}^{\prime} f^{\prime}}\left\{\left(\xi^{\prime}\right)_{\mathrm{ng}}+\frac{1}{\sqrt{2}}(H)_{\mathrm{pnb}}^{\dagger}\right\}}\right]\left[v_{\mathrm{NG}}^{(2) T} \phi_{0}^{(2)} e^{-\frac{i f_{1}^{\prime} \sqrt{2}}{f_{2}^{\prime} f^{\prime}}\left\{\left(\xi^{\prime}\right)_{\mathrm{ng}}+\frac{1}{\sqrt{2}}(H)_{\mathrm{pnb}}\right\}}\right]+h c
$$

Expanding exponential factors in the brackets in Eq. (151) and Eq. (152) to the second order, multiplying and adding quadratic and semiquadratic terms and setting further $\phi_{0}^{(1) T} v_{\mathrm{NG}}^{(1)}=v_{\mathrm{NG}}^{(1) T} \phi_{0}^{(1)}=f_{1}^{\prime 2}$, and also $\phi_{0}^{(2) T} v_{\mathrm{NG}}^{(2)}=v_{\mathrm{NG}}^{(2) T} \phi_{0}^{(2)}=f_{2}^{\prime 2}$ 
one finds the following quadratic equations for $V_{1}$ and $V_{2}$ respectively, neglecting constant potentials, as

$$
\begin{aligned}
V_{1}=\lambda^{(3)} 2\left(f_{1}^{\prime 2}\right)^{2}\left[\frac{2 f_{2}^{\prime 2}}{f_{1}^{\prime 2} f^{\prime 2}}\left\{\left(\xi^{\prime}\right)_{\mathrm{ng}}+\frac{1}{\sqrt{2}}(H)_{\mathrm{pnb}}^{\dagger}\right\}\left\{\left(\xi^{\prime}\right)_{\mathrm{ng}}+\frac{1}{\sqrt{2}}(H)_{\mathrm{pnb}}\right\}\right. \\
\left.-\frac{f_{2}^{\prime 2}}{f_{1}^{\prime 2} f^{\prime 2}}\left\{\left(\xi^{\prime}\right)_{\mathrm{ng}}+\frac{1}{\sqrt{2}}(H)_{\mathrm{pnb}}^{\dagger}\right\}^{2}-\frac{f_{2}^{\prime 2}}{f_{1}^{\prime 2} f^{\prime 2}}\left\{\left(\xi^{\prime}\right)_{\mathrm{ng}}+\frac{1}{\sqrt{2}}(H)_{\mathrm{pnb}}\right\}^{2}\right] \\
V_{2}=\lambda^{(3)} 2\left(f_{2}^{\prime 2}\right)^{2}\left[\frac{2 f_{1}^{\prime 2}}{f_{2}^{\prime 2} f^{\prime 2}}\left\{\left(\xi^{\prime}\right)_{\mathrm{ng}}+\frac{1}{\sqrt{2}}(H)_{\mathrm{pnb}}^{\dagger}\right\}\left\{\left(\xi^{\prime}\right)_{\mathrm{ng}}+\frac{1}{\sqrt{2}}(H)_{\mathrm{pnb}}\right\}\right. \\
\left.\quad-\frac{f_{1}^{\prime 2}}{f_{2}^{\prime 2} f^{\prime 2}}\left\{\left(\xi^{\prime}\right)_{\mathrm{ng}}+\frac{1}{\sqrt{2}}(H)_{\mathrm{pnb}}^{\dagger}\right\}^{2}-\frac{f_{1}^{\prime 2}}{f_{2}^{\prime 2} f^{\prime 2}}\left\{\left(\xi^{\prime}\right)_{\mathrm{ng}}+\frac{1}{\sqrt{2}}(H)_{\mathrm{pnb}}\right\}^{2}\right]
\end{aligned}
$$

Simplifying quadratic terms in the brackets in eqs. (153) and (154) one finds that NGBs vanish and leave PNB Higgs alone in the quadratic terms,

$$
V_{1}\left[V_{2}\right]=-\lambda^{(3)} \frac{f_{2}^{\prime 2} f_{1}^{\prime 2}}{f^{\prime 2}}\left[\frac{f_{1}^{\prime 2} f_{2}^{\prime 2}}{f^{\prime 2}}\right]\left\{(H)_{\mathrm{pnb}}^{\dagger}-(H)_{\mathrm{pnb}}\right\}^{2}=\lambda^{(3)}\left(\frac{f_{1}^{\prime 2} f_{2}^{\prime 2}}{f^{\prime 2}}\right) H^{\prime \prime \dagger} H^{\prime \prime}
$$

having made use the new field definition $H^{\prime \prime}=H^{\prime}-H$ and neglected $\operatorname{Tr}\left(H^{\prime \prime} H^{\prime \prime \dagger}\right)=$ $\frac{1}{2} v^{\prime \prime 2}$ with $\mathcal{O}\left(v^{\prime \prime}\right) \sim \mathcal{O}(100 \mathrm{GeV})$.

If the vanishing of NGBs has nothing to do with the newly emerging field $H^{\prime \prime}$ then it remains as PNB Higgs and gets the masses from $V_{1}$ and $V_{2}$ in Eq. (155), based on Eq. (148), with the mass-coupling as follows,

$$
\mu_{H_{i}^{\prime \prime}}^{2}=\left.\mu_{H_{i}^{\prime \prime}}^{2}\right|_{V_{1}}+\left.\mu_{H_{i}^{\prime \prime}}^{2}\right|_{V_{2}}=\lambda^{(3)} \frac{2 f_{1}^{\prime 2} f_{2}^{\prime 2}}{f^{\prime 2}}, i=1,2 .
$$

Nevertheless the righthand sides of Eq. (149) and Eq. (150) lefthand side resemble much with Eq. (115) after triplet-triplet splitting which provides either PNB Higgs or type-2B Scherk-Schwarz scalar with the same mass as shown at the righthand side, and the mass-squared is given in Eq. (157) below,

$$
m_{H_{i}^{\prime \prime}}^{2}=m_{H_{0 p}^{\prime \prime(i)}}^{2}=\frac{g^{\prime 4}}{16 \pi^{2}} \frac{\lambda^{(3)}}{f^{\prime 2}}\left(2 f_{1}^{\prime 2} f_{2}^{\prime 2}\right) \log \left(\frac{\Lambda_{(3)}^{2}}{\mu_{H_{i}^{\prime \prime}}^{2}}\right), i=1,2 .
$$

Another alternative for Coleman-Weinberg potential is given by the following,

$$
\begin{aligned}
& V^{\prime}=V_{1}^{\prime}+V_{2}^{\prime}, \\
& V_{1}^{\prime}=\lambda^{(3)}\left\{\phi^{(1) \dagger} \phi_{\mathrm{NG}}^{(2)} \phi_{\mathrm{NG}}^{(2) \dagger} \phi^{(1)}+\phi_{\mathrm{NG}}^{(2) \dagger} \phi^{(1)} \phi^{(1) \dagger} \phi_{\mathrm{NG}}^{(2)}\right\}, \\
& V_{2}^{\prime}=\lambda^{(3)}\left\{\phi^{(2) \dagger} \phi_{\mathrm{NG}}^{(1)} \phi_{\mathrm{NG}}^{(1) \dagger} \phi^{(2)}+\phi_{\mathrm{NG}}^{(1) \dagger} \phi^{(2)} \phi^{(2) \dagger} \phi_{\mathrm{NG}}^{(1)}\right\},
\end{aligned}
$$


where $V_{1}^{\prime}$ and $V_{2}^{\prime}$ can be rewritten as,

$$
\begin{aligned}
V_{1}^{\prime}= & \lambda^{(3)}\left[\phi_{0}^{(1) T} V_{\mathrm{NG}}^{(2)} e^{\frac{-i f_{1}^{\prime} \sqrt{2}}{f_{1}^{\prime} f^{\prime}}\left(-\frac{f_{1}^{\prime 2}}{f_{2}^{\prime 2}} \xi_{n g}^{\prime}+\frac{1}{\sqrt{2}}(H)_{\mathrm{pnb}}\right)}\right]\left[V_{\mathrm{NG}}^{(2) T} \phi_{0}^{(1)} e^{\frac{i f_{2}^{\prime} \sqrt{2}}{f_{1}^{\prime} f^{\prime}}\left(-\frac{f_{1}^{\prime 2}}{f_{2}^{\prime 2}} \xi_{n g}^{\prime}+\frac{1}{\sqrt{2}}(H)_{\mathrm{pnb}}^{\dagger}\right)}\right] \\
& +h c \\
V_{2}^{\prime}= & \lambda^{(3)}\left[\phi_{0}^{(2) T} V_{\mathrm{NG}}^{(1)} e^{\frac{i f_{1}^{\prime} \sqrt{2}}{f_{2}^{\prime} f^{\prime}}\left(-\frac{f_{2}^{\prime 2}}{f_{1}^{\prime 2}} \xi_{n g}^{\prime}+\frac{1}{\sqrt{2}}(H)_{\mathrm{pnb}}^{\dagger}\right)}\right]\left[V_{\mathrm{NG}}^{(1) T} \phi_{0}^{(2)} e^{\frac{-i f_{1}^{\prime} \sqrt{2}}{f_{2}^{\prime} f^{\prime}}\left(-\frac{f_{2}^{\prime 2}}{f_{1}^{\prime 2}} \xi_{n g}^{\prime}+\frac{1}{\sqrt{2}}(H)_{\mathrm{pnb}}\right)}\right] \\
& +h c .
\end{aligned}
$$

One obtains, after expanding exponents in the brackets in Eq. (161) and Eq. (162) to second order, multiplying and adding the quadratic and semiquadratic terms and setting further $\phi_{0}^{(1) T} V_{\mathrm{NG}}^{(2)}=V_{\mathrm{NG}}^{(2) T} \phi_{0}^{(1)}=\left(f_{1}^{\prime} f_{2}^{\prime}\right)$ and also $\phi_{0}^{(2) T} V_{\mathrm{NG}}^{(1)}=V_{\mathrm{NG}}^{(1) T} \phi_{0}^{(2)}=$ $\left(f_{1}^{\prime} f_{2}^{\prime}\right)$, the following quadratic equations,

$$
\begin{aligned}
& V_{1}^{\prime}=\lambda^{(3)} 2\left(f_{1}^{\prime} f_{2}^{\prime}\right)^{2}[ \frac{2 f_{2}^{\prime 2}}{f_{1}^{\prime 2} f^{\prime 2}}\left\{-\frac{f_{1}^{\prime 2}}{f_{2}^{\prime 2}} \xi_{n g}^{\prime}+\frac{1}{\sqrt{2}}(H)_{\mathrm{pnb}}\right\}\left\{-\frac{f_{1}^{\prime 2}}{f_{2}^{\prime 2}} \xi_{n g}^{\prime}+\frac{1}{\sqrt{2}}(H)_{\mathrm{pnb}}^{\dagger}\right\} \\
&-\frac{f_{2}^{\prime 2}}{f_{1}^{\prime 2} f^{\prime 2}}\left\{-\frac{f_{1}^{\prime 2}}{f_{2}^{\prime 2}} \xi_{n g}^{\prime}+\frac{1}{\sqrt{2}}(H)_{\mathrm{pnb}}\right\}^{2} \\
&\left.-\frac{f_{2}^{\prime 2}}{f_{1}^{\prime 2} f^{\prime 2}}\left\{-\frac{f_{1}^{\prime 2}}{f_{2}^{\prime 2}} \xi_{n g}^{\prime}+\frac{1}{\sqrt{2}}(H)_{\mathrm{pnb}}^{\dagger}\right\}^{2}\right], \\
& V_{2}^{\prime}=\lambda^{(3)} 2\left(f_{1}^{\prime} f_{2}^{\prime}\right)^{2}\left[\frac{2 f_{1}^{\prime 2}}{f_{2}^{\prime 2} f^{\prime 2}}\left\{-\frac{f_{2}^{\prime 2}}{f_{1}^{\prime 2}} \xi_{n g}^{\prime}+\frac{1}{\sqrt{2}}(H)_{\mathrm{pnb}}^{\dagger}\right\}\left\{-\frac{f_{2}^{\prime 2}}{f_{1}^{\prime 2}} \xi_{n g}^{\prime}+\frac{1}{\sqrt{2}}(H)_{\mathrm{pnb}}\right\}\right. \\
&-\frac{f_{1}^{\prime 2}}{f_{2}^{\prime 2} f^{\prime 2}}\left\{-\frac{f_{2}^{\prime 2}}{f_{1}^{\prime 2}} \xi_{n g}^{\prime}+\frac{1}{\sqrt{2}}(H)_{\mathrm{pnb}}^{\dagger}\right\}^{2} \\
&\left.-\frac{f_{1}^{\prime 2}}{f_{2}^{\prime 2} f^{\prime 2}}\left\{-\frac{f_{2}^{\prime 2}}{f_{1}^{\prime 2}} \xi_{n g}^{\prime}+\frac{1}{\sqrt{2}}(H)_{\mathrm{pnb}}\right\}^{2}\right] .
\end{aligned}
$$

Simplying quadratic terms in the brackets in Eq. (163) and Eq. (164) one obtains finally the vanishing of NGBs and the potential as follows,

$$
V_{1}^{\prime}\left[V_{2}^{\prime}\right]=-\lambda^{(3)} \frac{f_{2}^{\prime 4}}{f^{\prime 2}}\left[\frac{f_{1}^{\prime 4}}{f^{\prime 2}}\right]\left\{(H)_{\mathrm{pnb}}^{\dagger}-(H)_{\mathrm{pnb}}\right\}^{2}=\lambda^{(3)} \frac{f_{2}^{\prime 4}\left[f_{1}^{\prime 4}\right]}{f^{\prime 2}} H^{\prime \prime \dagger} H^{\prime \prime},
$$

where $H^{\prime \prime}=H^{\prime}-H, \operatorname{Tr}\left(H^{\prime \prime} H^{\prime \prime \dagger}\right)=\frac{1}{2} v^{\prime \prime 2}$ with $\mathcal{O}\left(v^{\prime \prime}\right) \sim \mathcal{O}(100 \mathrm{GeV})$ to be neglected. Based on Eq. (158) the mass-coupling can be obtained directly from Eq. (165), which represents the mass of Heisenberg scalar $H_{H}^{\prime \prime}$, based on resemblance between Eq. (159), Eq. (160) and Eq. (119) facilitating for Higgs-NGB unification with additional mass and degrees of freedom, as follows,

$$
\mu_{H_{H}^{\prime \prime}}^{2}=\left.\mu_{H_{H}^{\prime \prime}}^{2}\right|_{V_{1}^{\prime}}+\left.\mu_{H_{H}^{\prime \prime}}^{2}\right|_{V_{2}^{\prime}}=\frac{\lambda^{(3)}}{f^{\prime 2}}\left(f_{1}^{\prime 4}+f_{2}^{\prime 4}\right)
$$


and the mass-squared as provided by eq. (167) respectively,

$$
m_{H_{H}^{\prime \prime}}^{2}=\frac{g^{\prime 4}}{16 \pi^{2}} \frac{\lambda^{(3)}}{f^{\prime 2}}\left(f_{1}^{\prime 4}+f_{2}^{\prime 4}\right) \log \left(\frac{\Lambda_{(3)}^{2}}{\mu_{H_{H}^{\prime \prime}}^{2}}\right),
$$

where $H_{H}^{\prime \prime}$ Heisenberg scalar, $\mathcal{O}\left(\mu_{H_{i}^{\prime \prime}}\right) \sim \mathcal{O}\left(\mu_{H_{H}^{\prime \prime}}\right) \sim \mathcal{O}(100 \mathrm{GeV})$ and $\mathcal{O}\left(\Lambda_{(3)}\right) \sim$ $\mathcal{O}(100 \mathrm{TeV})$. Eq. (157) and Eq. (167) facilitate the formation of exotic scalars in $\mathrm{SU}(3)$ level consisting of 3-scalar Higgs and 3-component scalars as previously discussed above.

Next, we discuss some phenomenological aspects especially on the mass spectrum of PNB Higgs, 3-scalar Higgs, Heisenberg and Scherk-Schwarz scalars including exotic 3-component scalars.

\subsection{Phenomenological Aspects}

\subsubsection{The Order of $\mathrm{SU}(3) \times \mathrm{SU}(3)$ cut-off scale}

Some phenomenological aspects within the current model are briefly examined. That is, the order estimation for the cut-off scale.

First of all, let us perform the order estimation for the cut-off scale $\Lambda_{(3)}$. The contribution of quadratically divergent one-loop diagram to the Higgs mass in the conventional Simplest Little Higgs $\mathrm{SU}(3) \times \mathrm{SU}(3) \times \mathrm{U}(1)$ is given by the first part of Eq. (168) $[29,30]$ and, after performing a little calculation, one finds the second part at the righthand as

$$
\sim \frac{g^{\prime 2}}{16 \pi^{2}}\left(\Lambda^{(0)}\right)^{2} \operatorname{Tr}\left(\phi^{(1) \dagger} \phi^{(1)}+\phi^{(2) \dagger} \phi^{(2)}\right) \text { or } \sim \frac{g^{\prime 2}}{16 \pi^{2}}\left(\Lambda^{(0)}\right)^{2} \times 3,
$$

where $\Lambda^{(0)}$ is the cut-off scale at the conventional Simplest Little Higgs. Hence, the cut-off scale at the present theory can be estimated to be $\Lambda_{(3)}>\sqrt{3} \Lambda^{(0)}$. On the other hand, from $[30,32]$ the cut-off scale $\Lambda^{(0)}$ is around $10 \mathrm{Te} V$ with $\operatorname{VEV} f^{(0)} \sim 1$ $\mathrm{Te} V$. It is also related to the SM cut-off $M_{\text {weak }} \sim 80 \mathrm{Ge} V$ through $\Lambda^{(0)} \sim 4 \pi f^{(0)} \sim$ $(4 \pi)^{2} M_{\text {weak }}$. From above one can set, $\sqrt{3} \Lambda^{(0)}<\Lambda_{(3)}<4 \pi f^{\prime}$, then the cut-off scale $\Lambda_{(3)}$ within the present model, taking the upper limit, $\sim 100 \mathrm{Te} V$ for $f^{\prime} \sim 10 \mathrm{Te} V$. Therefore, the cut-off scale and VEV at the present scenario is higher than the ones in the conventional Simplest Little Higgs scenario.

\subsubsection{Higgs spectrum and masses}

Three Higgs bosons emerge as the beyond-SM Higgses of SU(3) level with masses shown successively in Eq. (157), for a scalar-pair of light Higgs bosons and Eq. (143) for light 3-scalar Higgs bosons. On the other side some exotic scalars show up consisting of a Heisenberg scalar, a scalar-pair of type-2B Scherk-Schwarz scalars, and two 3-component scalars (Higgs-like and gauge-like) with the masses given in Eq. (167), Eq. (157) and Eq. (143) respectively. If one sets $f_{1}^{\prime} \sim 4.0 \mathrm{Te} V$, and $f_{2}^{\prime} \sim 5.0 \mathrm{Te} V, g^{\prime}=0.6$ and $\lambda^{(3)} \sim 0.1$ with cut-off scale $\Lambda_{(3)} \sim 10 \mathrm{Te} V$ as above and $\mu_{H^{\prime \prime}} \sim 100 \mathrm{GeV}$ then mass of Higgs is $79 \mathrm{GeV}$ for light Higgs $H_{1}^{\prime \prime}$ and $H_{2}^{\prime \prime}$ while 
mass of 3-scalar Higgs boson $H_{i}^{\prime \prime}-H_{H}^{\prime \prime}-H_{i}^{\prime \prime}, m_{H_{3 h}^{\prime \prime}} \sim 139 \mathrm{GeV}$ with Heisenberg scalar, $m_{H_{H}^{\prime \prime}} \sim 114 \mathrm{GeV}$ and type-2B Scherk-Schwarz scalar $m_{H_{o p}^{\prime \prime}} \sim 79 \mathrm{GeV}$. It means that the gauge-like single scalar from $\mathrm{SU}(6)$-origin $H_{0}^{\prime \prime}$ has the mass of $\sim 114$ $\mathrm{GeV}$ at this level. On the other side Eq. (143) provides mass of Higgs-like or gaugelike 3-component scalar, $H_{H}^{\prime \prime}-H_{0 p(1)}^{\prime \prime}=H_{0 p(2)}^{\prime \prime}, H_{0 p(1)}^{\prime \prime}-H_{H}^{\prime \prime}-H_{o p(2)}^{\prime \prime}, m_{H_{o p-H-o p}^{\prime \prime}} \sim$ $m_{H_{H-20 p}^{\prime \prime}} \sim 160 \mathrm{GeV}$.

On the other hand if VEV s are set at $f_{1}^{\prime}=16 \mathrm{Te} V, f_{2}^{\prime}=20.0 \mathrm{Te} V$ and $\Lambda_{(3)} \sim 100$ $\mathrm{TeV}$ and other parameters remain the same then one finds mass of heavy 3-scalar Higgs to be $567 \mathrm{GeV}$, still below unitary constraint $\sim 700 \mathrm{GeV}$ while Eq. (157) excludes the light Higgs boson $m_{H_{i}^{\prime \prime}} \sim 392 \mathrm{GeV}$. This reconfirms that Eq. (157) is valid for light Higgs while Eq. (143) for both light and heavy 3-scalar Higgses with the excluded region in-between the light and the heavy lies, based on latest LHC data, in the interval $145-466 \mathrm{GeV}$. The large Heisenberg mass is found to be $411 \mathrm{GeV}$ with $\operatorname{VEV} \mathcal{O}\left(f_{i}^{\prime}\right) \sim \mathcal{O}(10 \mathrm{Te} V)$ which is excluded from free Higgs region but, of course, included in free gauge-like scalar region (up to $1.5 \mathrm{TeV}$ ) [18]. Thus (being together with PNB), Heisenberg scalar always lives in a small confined space of 3-scalar Higgs.

Masses of gauge-like 3-component scalar and Higgs-like 3-component scalar are basically in-between 1-3 times of the mass-squared for the Heisenberg scalar. Masses of both types are the same of the first $m_{H_{0 p-H-0 p}^{\prime \prime}}$, becoming $\sim 690 \mathrm{GeV}$ with a single type-2B Scherk-Schwarz scalar $m_{H_{0 p}^{\prime \prime}} \sim 392 \mathrm{GeV}$ while of the second $m_{H_{H-20 p}^{\prime \prime}} \sim 690$ $\mathrm{GeV}$ which are within the range of heavy Higgs mass. As a free particle type-2A Scherk-Schwarz scalar becomes a gauge-like single scalar with the same mass (411 $\mathrm{GeV}$ ) but as bonded particles the heavy type-2B Scherk-Schwarz scalar and heavy Heisenberg scalar with masses of $392 \mathrm{GeV}$ and $411 \mathrm{GeV}$ successively. The heaviest types of 3-component scalars take on the forms $H_{0-H-0}^{\prime \prime}$ and $H_{H-20}^{\prime \prime}$ with the mass $m_{H_{0}^{\prime \prime}} \sqrt{3}$ following Eq. (143) or $712 \mathrm{GeV}$. Maximum mass of gauge-like 3-component scalar can be approximated with $f^{\prime}=f_{i}^{\prime} \sqrt{2}$ where it is found $f_{i}^{\prime} \sim 70 \mathrm{TeV}$ which gives the required mass of $1.55 \mathrm{TeV}$.

\section{Conclusion}

The equality between the infinitesimal gauge parameter of SU(6) and infinitesimal extra-dimensional rotation angle of $S^{1} / Z_{2}$ brings the correspondence between the broken fifth component of $5 \mathrm{D}$ gauge boson and $5 \mathrm{D}$-twist NGB in the $5 \mathrm{D}(y \sim 0)$ near-brane. The replacement of the first in Coleman-Weinberg potential with the second changes the potential becoming a generalized Coleman-Weinberg potential which facilitates the unification of Higgs and NGB. This completes the triangle of unification formed by Higgs-(5D)gauge boson (4D)-NGB with the first line shows 5D gauge-Higgs unification principles and the second represents the unification (eating) of NGB with (by) a 4D massless gauge boson in electroweak Higgs mechanism while the third is the unification of Higgs and NGBs realized through the swallowing and digesting mode.

The Higgs-NGB unification is conserved under unitary transformation and also 
in the $\mathrm{SU}(6)$ and $\mathrm{SU}(3)$ symmetry breakings so that a massive Heisenberg (type I Scherk-Schwarz) scalar, $H_{H}^{\prime \prime}$, exists under $\mathrm{SU}(6)$ and $\mathrm{SU}(3)$ breakings i.e. as a bonded scalar after $\mathrm{SU}(6)$ breaking and a free scalar after $\mathrm{SU}(3)$ breaking. On the other side the type-IIA Scherk-Schwarz scalar of SU(6)-origin $H_{0}^{\prime \prime}$ gets the mass after triplet-triplet splitting and becomes a stand-alone free scalar i.e. a gaugelike single scalar which must decouple from SU(3) level. The SU(6)-origin massive Heisenberg scalar, after triplet-triplet splitting, is basically accompanied by massive SU(6)-origin PNB Higgs $\left(H_{i}^{\prime \prime}\right)$ and Scherk-Schwarz scalar of type-2B $\left(H_{0 p}^{\prime \prime}\right)$. Therefore Heisenberg scalar, here, is bonded by 2 (two) PNB Higgses or type-2B Scherk-Schwarz scalars forming the 3-scalar Higgs or the Higgs-like (gauge-like) 3component scalar as exotic scalars produced from twistedly-broken shift symmetry breaking.

On the other side massive Heisenberg scalar and type-2B Scherk-Schwarz scalar are also produced from $\mathrm{SU}(3) \times \mathrm{SU}(3) \times \mathrm{U}(1)$ breaking as free scalars which can be called as gauge-like single scalars. These are results of SU(3) twistedly-broken shift symmetry breaking. Apart from the above scalars there is also $\mathrm{SU}(6)$-origin, being still massless through triplet-triplet splitting, PNB Higgs $\left(H\right.$ and $\left.H^{\prime}\right)$ being unified with NGBs in $H_{0}^{\prime \prime}$ which is produced originally from the $\mathrm{SU}(6)$ shiftedly-broken shift symmetry breaking. Now, due to twistedly-broken $\mathrm{SU}(3)$ shift symmetry breaking $H_{0}^{\prime \prime}$ decomposes so that one finds the breaking of a $\mathrm{SU}(3)$ free gauge-like single scalar becoming two PNB Higgses, $H$ and $H^{\prime}$, and releasing NGB through extradimensional twist i.e decoupling from the lower-near-brane.

These exotic scalars and its properties pose certainly a new challenge for the research and demand seriously a great effort to unveil. Perhaps, it can still be done in LHC for the next decade.

\section{Acknowledgements}

AH would like to thank ICTMP and PT Enerfra Septadaya Prima for administrative support while FPZ to Institut Teknologi Bandung and Dirjen Dikti for Research fund (fiscal year 2013).

\section{Appendix}

\section{A The factor of double-vacua shift symmetry and its Scherk-Schwarz (SS-) twist breaking terms by means of $\mathrm{BCH}$ expansion}

Coleman-Weinberg potential can be written in general quartic form as,

$$
\begin{aligned}
& V_{c w}(i=j)[i \neq j] \\
& =\left\{v^{T}\left[v^{\prime T}\right] e^{a_{i}^{*} A^{\dagger}} v\left[v^{\prime}\right]\left[v^{\prime}[v]\right] e^{b_{j} B}\right\}\left\{v^{T}\left[v^{\prime T}\right]\left[v^{\prime T}\left[v^{T}\right]\right] e^{b_{j}^{*} B^{\dagger}} v\left[v^{\prime}\right] e^{a_{i} A}\right\} \\
& \quad+\left\{v^{T}\left[v^{T}\right]\left[v^{\prime T}\left[v^{T}\right]\right] e^{b_{j}^{*} B^{\dagger}} v\left[v^{\prime}\right] e^{a_{i} A}\right\}\left\{v^{T}\left[v^{\prime T}\right] e^{a_{i}^{*} A^{\dagger}} v\left[v^{\prime}\right]\left[v^{\prime}[v]\right] e^{b_{j} B}\right\},
\end{aligned}
$$


defining $V_{c w}=V_{c w}(i=j)[i \neq j], i=1,2$ with $i[j]$ corresponds to $v\left(v^{T}\right)$ and $a_{i}\left(a_{i}^{*}\right) A\left(A^{\dagger}\right)\left[v^{\prime}\left(v^{\prime T}\right)\right.$ and $\left.b_{j}\left(b_{j}^{*}\right) B\left(B^{\dagger}\right)\right]$, Eq. (169) is rewritten, omitting temporarily coefficient $a_{i}\left(a_{i}^{*}\right)$ and $b_{j}\left(b_{j}^{*}\right)$ for simplicity, as

$$
\begin{aligned}
V_{c w}= & V_{c w}(i=j)[i \neq j] \\
= & \left(v^{T} v\right)^{2}\left[\left(v^{T}\left[v^{\prime}\right]\right)^{2}\right]\left[\left(\left[v^{\prime T} v^{\prime}\right]\right)^{2}\left[\left(v^{T}[v]\right)^{2}\right]\right]\left(e^{A^{\dagger}} e^{B}\right)\left(e^{B^{\dagger}} e^{A}\right) \\
& \left.+\left(v^{T} v\right)^{2}\left[\left(\left[v^{T}\right] v\right)\right)^{2}\right]\left[\left(\left[v^{\prime T} v^{\prime}\right]\right)^{2}\left[\left(\left[v^{T}\right] v^{\prime}\right)^{2}\right]\right]\left(e^{B^{\dagger}} e^{A}\right)\left(e^{A^{\dagger}} e^{B}\right) .
\end{aligned}
$$

Defining the following

$$
F=\left(e^{A^{\dagger}} e^{B}\right)\left(e^{B^{\dagger}} e^{A}\right)+\left(e^{B^{\dagger}} e^{A}\right)\left(e^{A^{\dagger}} e^{B}\right)
$$

one rewrites Coleman-Weinberg potential as,

$$
V_{c w}=V_{c w}(i=j)[i \neq j]=\left(v^{T} v\right)^{2}\left[\left(v^{T}\left[v^{\prime}\right]\right)^{2}\right]\left[\left(\left[v^{\prime T} v^{\prime}\right]\right)^{2}\left[\left(v^{T}[v]\right)^{2}\right]\right] F,
$$

where $v^{T} v^{\prime}=v^{\prime T} v$ has been applied. In the generalized form $A, B$ are operators or operator-like generators which must be treated via Baker-Campbell-Hausdorff (BCH) formula as follows $[31,45]$,

$$
\begin{aligned}
& e^{A^{\dagger}} e^{B}=e^{A^{\dagger}+B+\frac{1}{2}\left[A^{\dagger}, B\right]}, \\
& e^{B^{\dagger}} e^{A}=e^{B^{\dagger}+A+\frac{1}{2}\left[B^{\dagger}, A\right]},
\end{aligned}
$$

which can be expanded to the lowest order as below,

$$
\begin{aligned}
& e^{A^{\dagger}} e^{B} \sim 1+A^{\dagger}+B+\frac{1}{2}\left[A^{\dagger}, B\right], \\
& e^{B^{\dagger}} e^{A} \sim 1+B^{\dagger}+A+\frac{1}{2}\left[B^{\dagger}, A\right] .
\end{aligned}
$$

Consequently $F$-factor can be expressed as,

$$
\begin{aligned}
F \sim & \left(1+A^{\dagger}+B+\frac{1}{2}\left[A^{\dagger}, B\right]\right)\left(1+B^{\dagger}+A+\frac{1}{2}\left[B^{\dagger}, A\right]\right) \\
& +\left(1+B^{\dagger}+A+\frac{1}{2}\left[B^{\dagger}, A\right]\right)\left(1+A^{\dagger}+B+\frac{1}{2}\left[A^{\dagger}, B\right]\right) .
\end{aligned}
$$

After some calculation one obtains as follows,

$$
\begin{aligned}
& F \sim\left\{1+\left(A^{\dagger}+B^{\dagger}\right)+(A+B)+\frac{1}{2}\left(\left[B^{\dagger}, A\right]+\left[A^{\dagger}, B\right]\right)+\left(A^{\dagger} B^{\dagger}+B A\right)\right. \\
& +\left(A^{\dagger} A+B B^{\dagger}\right) \frac{1}{2}\left(A^{\dagger}\left[B^{\dagger}, A\right]+\left[A^{\dagger}, B\right] B^{\dagger}\right)+\frac{1}{2}\left(B\left[B^{\dagger}, A\right]+\left[A^{\dagger}, B\right] A\right) \\
& \left.+\frac{1}{4}\left[A^{\dagger}, B\right]\left[B^{\dagger}, A\right]\right\}\left\{1+(A+B)+\left(A^{\dagger}+B^{\dagger}\right)+\frac{1}{2}\left(\left[A^{\dagger}, B\right]+\left[B^{\dagger}, A\right]\right)\right. \\
& +\left(A B+B^{\dagger} A^{\dagger}\right)+\left(A A^{\dagger}+B^{\dagger} B\right) \frac{1}{2}\left(A\left[A^{\dagger}, B\right]+\left[B^{\dagger}, A\right] B\right) \\
& \left.+\frac{1}{2}\left(B^{\dagger}\left[A^{\dagger}, B\right]+\left[B^{\dagger}, A\right] A^{\dagger}\right)+\frac{1}{4}\left[B^{\dagger}, A\right]\left[A^{\dagger}, B\right]\right\} \\
& \sim 2+2\left(A^{\dagger}+B^{\dagger}\right)+2(A+B)+\left(\left[B^{\dagger}, A\right]+\left[A^{\dagger}, B\right]\right)+\left(A^{\dagger} B^{\dagger}+B^{\dagger} A^{\dagger}\right)+(A B+B A) \\
& +\left(A^{\dagger} A+A A^{\dagger}\right)+\left(B^{\dagger} B+B B^{\dagger}\right)+\frac{1}{2}\left\{\left(A^{\dagger}+B\right)\left[B^{\dagger}, A\right]+\left[B^{\dagger}, A\right]\left(B+A^{\dagger}\right)\right\} \\
& +\frac{1}{2}\left\{\left[A^{\dagger}, B\right]\left(B^{\dagger}+A\right]+\left(A+B^{\dagger}\right)\left[A^{\dagger}, B\right]\right\}+\frac{1}{2}\left[A^{\dagger}, B\right]\left[B^{\dagger}, A\right],
\end{aligned}
$$


which can be rewritten into a more compact form by means of anti-commutator as,

$$
\begin{aligned}
F \sim & 2+2\left(A^{\dagger}+B^{\dagger}\right)+2(A+B)+\left(\left[B^{\dagger}, A\right]-\left[B, A^{\dagger}\right]\right)+\left\{A^{\dagger}, B^{\dagger}\right\}+\{A, B\} \\
& +\left\{A^{\dagger}, A\right\}+\left\{B^{\dagger}, B\right\}+\frac{1}{2}\left\{\left(A^{\dagger}+B\right),\left[B^{\dagger}, A\right]\right\}+\frac{1}{2}\left\{\left[A^{\dagger}, B\right],\left(A+B^{\dagger}\right)\right\} \\
& +\frac{1}{2}\left[A^{\dagger}, B\right]\left[B^{\dagger}, A\right]
\end{aligned}
$$

Considering terms of $\mathrm{BCH}$ expansion up to first order with only commutators, and anti-commutators of 2 (two) operators only, and neglecting the second order and third order such as (anti)-commutators with 3 (three) and 4 (four) operators, and also defining $F=2 F_{6}\left[F_{3}\right]$ Eq. (178) is reexpressed finally for $\mathrm{SU}(6)$ and $\mathrm{SU}(3)$ as follows,

$$
\begin{aligned}
F_{6}\left[F_{3}\right]= & 1+\left(A^{\dagger}+B^{\dagger}\right)+(A+B)+\frac{1}{2}\left(\left[B^{\dagger}, A\right]-\left[B, A^{\dagger}\right]\right)+ \\
& \frac{1}{2}\left(\left\{A^{\dagger}, B^{\dagger}\right\}+\{A, B\}\right)+\frac{1}{2}\left(\left\{A^{\dagger}, A\right\}+\left\{B^{\dagger}, B\right\}\right) .
\end{aligned}
$$

Factor- $F_{6}\left[F_{3}\right]$ reflects the double-vacua shift symmetry and its SS-twist main breaking terms. A special notice must be given to Eq.(A.10), under unitary transformation, the second and third order may still provide a significant influence especially under infinitesimal unitary transformation.

\section{B The Unitarily-transformed trace of unitary matrix for scalar (Higgs) potential}

The constancy of the trace of unitary matrix under unitary transformation is to be verified below. Scalar (Higgs) potential must be constant under unitary transformation but be changed under symmetry breaking if kinetic terms turn out to be fixed. The $\mathrm{SU}(6)[\mathrm{SU}(3)]$ gauge invariance of the Lagrangian can be kept fixed only if the scalar (Higgs) potential is built from $\mathrm{SU}(6)$ [SU(3)]-invariant functions which can be formed from powers of the scalar (Higgs) field. In most general form this potential can be written as [45],

$$
V(\Phi)[\phi]=-\frac{1}{2} \mu^{2} \operatorname{Tr}\left(\Phi^{2}\left[\phi^{2}\right]\right)+a\left\{\operatorname{Tr}\left(\Phi^{2}\left[\phi^{2}\right]\right)\right\}^{2}+b\left\{\operatorname{Tr}\left(\Phi^{4}\left[\phi^{4}\right]\right)\right\},
$$

where $\Phi[\phi]$ is the scalar (Higgs) field of $\mathrm{SU}(6)[\mathrm{SU}(3)], \mu$ the coupling contant, $a$ and $b$ the constants. Potential $V(\Phi)[\phi]$ in Eq.(B.1) is constant under unitary transformation if the trace of unitary matrix doest not change under such transformation. Generalizing $\Phi^{2}\left[\phi^{2}\right], \Phi^{4}\left[\phi^{4}\right]$ to $\Phi^{n}\left[\phi^{n}\right], n=1,2,3 \ldots, 4$ and applying $\operatorname{Tr}(A B)=\operatorname{Tr}(B A)$ one calculates the trace of power-n unitary matrix of scalar (Higgs) field and obtains [45],

$$
\begin{aligned}
\operatorname{Tr}\left(\Phi^{\prime n}\right) & =\operatorname{Tr}\left\{\left(U \Phi U^{\dagger}\right)^{n}\right\}=\operatorname{Tr}\left\{U \Phi U^{\dagger} U \Phi U^{\dagger} \ldots U \Phi U^{\dagger}\right\} \\
& =\operatorname{Tr}\left\{\left(U \Phi^{n} U^{\dagger}\right)\right\}, \text { due to } U^{\dagger} U=U U^{\dagger}=1 \\
& =\operatorname{Tr}\left(\Phi^{n}\right), \text { from } U^{\dagger} U \Phi^{n} U^{\dagger} U
\end{aligned}
$$


from which scalar product or its trace is constant $(C)$ under unitary transformation as below,

$$
\operatorname{Tr}\left(\Phi^{\prime \dagger} \Phi^{\prime}\right)=\operatorname{Tr}\left(\Phi^{\dagger} \Phi\right)=C
$$

\section{The Exotic scalar's schematic drawings}
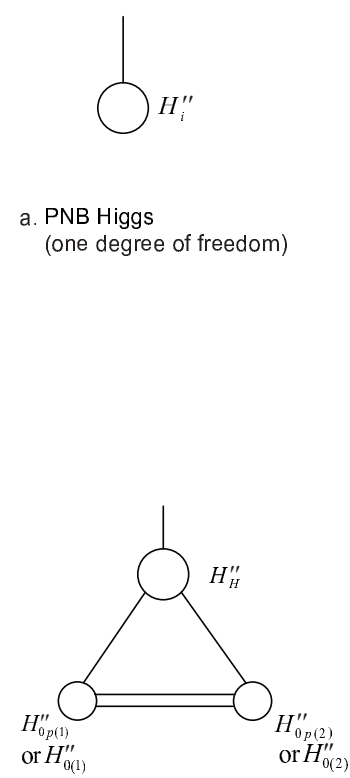

c. Higgs-like 3-component scalar (one free degree of freedom)

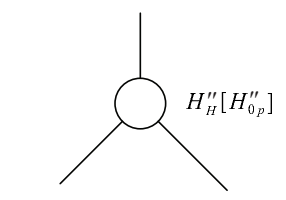

b. Heisenberg scalar

(Scherk-Schwarz scalar of type-I)- (3 (three) degrees of freedom), or Scherk-Schwarz scalar of type IIB (3 degrees of freedom)

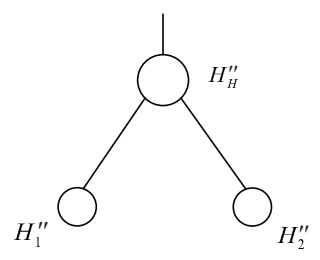

d. 3-scalar Higgs (one degree of freedom)
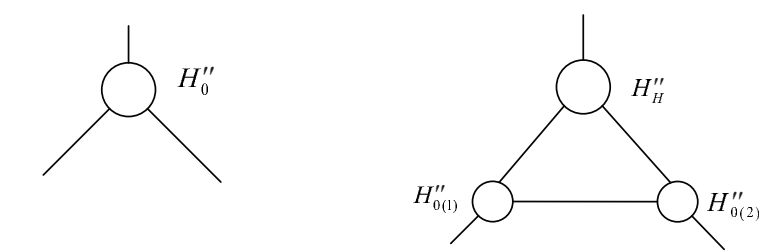

e. Scherk-Schwarz scalar of type II A (3 degrees of freedom)

\section{f. Gauge-like 3-component scalar (3 (three) degrees} of freedom)

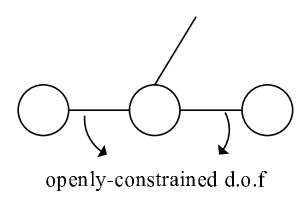

d.o.f : degrre of freedom

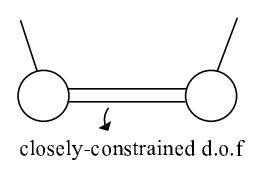

D Strong approach for strongly-coupled scalar in SU(6)

Consider SU(6) scalar fields as follows

$$
\widetilde{\Phi}^{(1)}=v e^{\frac{i f_{2}}{f_{1}} \theta}, \quad \widetilde{\Phi}^{(2)}=v^{\prime} e^{-\frac{i f_{1}}{f_{2}} \theta},
$$


where the following VEVs and LittleHiggs-like scalar $\theta$ have been defined as,

$$
\left.v=\left(\begin{array}{c}
0 \\
0 \\
f_{1} \\
0 \\
0 \\
0
\end{array}\right), \quad v^{\prime}=\left(\begin{array}{c}
0 \\
0 \\
0 \\
0 \\
0 \\
f_{2}
\end{array}\right), \quad \theta=\frac{1}{f}\left(\begin{array}{ccc}
(0)_{3 \times 3} \\
0 & 0 & \left(h^{\prime}\right)_{2 \times 1} \\
0 & 0 & 0 \\
\left(h^{\dagger}\right)_{1 \times 2} & 0
\end{array}\right), \quad \begin{array}{ccc}
0 & 0 & (h)_{2 \times 1} \\
0 & 0 & \\
\left(h^{\prime \dagger}\right)_{1 \times 2} & 0
\end{array}\right),
$$

with $\epsilon=\frac{1}{f}$ a very small number, $h$ complex scalar field which can be further defined as,

$$
h=h_{1}-i H, \quad h^{\prime}=h_{1}^{\prime}-i H^{\prime}, \quad H\left[H^{\prime}\right] \gg h_{1}\left[h_{1}^{\prime}\right]
$$

Eq. (185) dictates the emerging of two $\theta$-parameters namely $\theta_{h_{1}}$ and $\theta_{H}$ where $\theta=\theta_{h_{1}}+\theta_{H}$ which are defined accordingly as follows,

$$
\begin{aligned}
& \theta_{h_{1}}\left[\theta_{H}\right]= \\
& \frac{1}{f}\left(\begin{array}{ccc}
(0)_{3 \times 3} & \left(\begin{array}{ccc}
0 & 0 & \\
0 & 0 & \left(h_{1}\right)_{2 \times 1}[(i H)]_{2 \times 1} \\
\left(h_{1}^{\prime \dagger}\right)_{1 \times 2}\left[\left(i H^{\prime \dagger}\right)\right]_{1 \times 2} & 0
\end{array}\right) \\
0 & 0 & \left(h_{1}^{\prime}\right)_{2 \times 1}\left[\left(i H^{\prime}\right)\right]_{2 \times 1} \\
0 & 0 & 0
\end{array}\right),
\end{aligned}
$$

So that Eq. (183) is rewritten as below,

$$
\widetilde{\Phi}^{(1)}=v e^{\frac{i f_{2}}{f_{1}} \theta_{h_{1}}} e^{\frac{i f_{2}}{f_{1}} \theta_{H}}, \quad \widetilde{\Phi}^{(2)}=v^{\prime} e^{-\frac{i f_{1}}{f_{2}} \theta_{h_{1}}} e^{-\frac{i f_{1}}{f_{2}} \theta_{H}},
$$

From definitions in Eq. (185) it is clear that $\theta_{h_{1}}$ is a hermitian matrix which further provides $e^{\frac{i f_{2}}{f_{1}} \theta_{h_{1}}}\left[e^{-\frac{i f_{1}}{f_{2}} \theta_{h_{1}}}\right]$ as unitary matrix in the unitary transformation. These factors, as we have known, creates mass matrix in the weak coupling realm and one can neglect in the pursuit of LittleHiggs-like scalar characters in the strong coupling. Now, one has the following

$$
\widetilde{\Phi}^{(1)^{\prime}}=v e^{\frac{i f_{2}}{f_{1}} \theta_{H}}, \quad \widetilde{\Phi}^{(2)^{\prime}}=v^{\prime} e^{-\frac{i f_{1}}{f_{2}} \theta_{H}},
$$

which can be rewritten, making use the definition below,

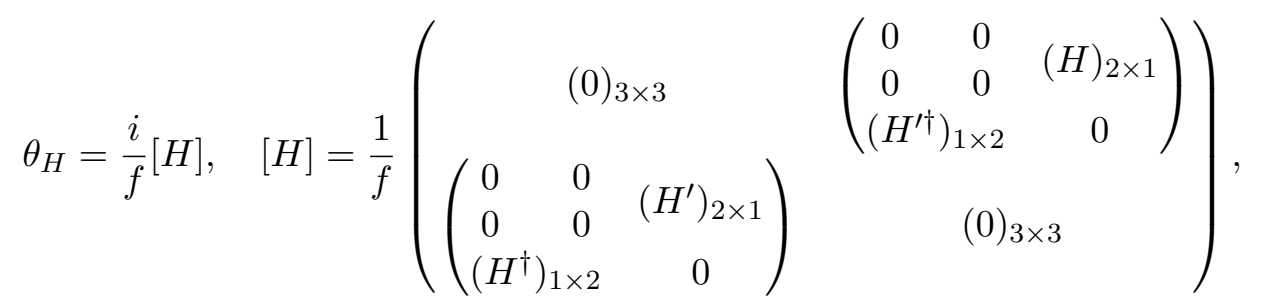


as follows,

$$
\widetilde{\Phi}^{(1)^{\prime \prime}}=v e^{-\frac{f_{2}}{f_{1} f}[H]}, \quad \widetilde{\Phi}^{(2)^{\prime \prime}}=v^{\prime} e^{\frac{f_{1}}{f_{2} f}[H]},
$$

where $H$ and $H^{\prime}$ are real scalar fields so that matrix $[\mathrm{H}]$ is hermitian. Eq. (190) represents the strongly-coupled effect which will be discussed further especially its role in the unitary matrix. Simplifying the exponents by neglecting factors $\frac{f_{2}}{f_{1}}\left[\frac{f_{1}}{f_{2}}\right]$ for the moment and utilising $\epsilon=\frac{1}{f}$, one can expand the exponent as follows,

$$
e^{ \pm \epsilon[H]} \sim 1 \pm \epsilon[H]+\frac{\epsilon^{2}}{2}[H]^{2} \pm \frac{\epsilon^{3}}{3 !}[H]^{3}+\frac{\epsilon^{4}}{4 !}[H]^{4} \pm \frac{\epsilon^{5}}{5 !}[H]^{5}+\frac{\epsilon^{6}}{6 !}[H]^{6}+\cdots
$$

which consist of two series $H_{2 n}$ and $H_{2 n+1}$ for even and odd powers respectively. One can also approach both series by setting limiting with its terms behave as the upper bound terms,

$$
\begin{aligned}
H_{2 n} & =1+\frac{\epsilon^{2}}{2}[H]^{2}+\frac{\epsilon^{4}}{4 !}[H]^{4}+\frac{\epsilon^{6}}{6 !}[H]^{6}+\cdots \\
& \sim 1+\frac{\epsilon^{2}}{2}[H]^{2}+\frac{1}{3}\left(\frac{1}{2 !} \frac{\epsilon^{4}}{4}[H]^{4}\right)+\frac{1}{15}\left(\frac{1}{3 !} \frac{\epsilon^{6}}{8}[H]^{6}\right) \\
& <e^{\frac{\epsilon}{2}\left[H^{2}\right]} \sim 1\left(\text { due to } \epsilon \ll 1, H\left[H^{\prime}\right] \ll h_{1}\right) \\
H_{2 n+1} & = \pm \epsilon[H] \pm \frac{\epsilon^{3}}{3 !}[H]^{3} \pm \frac{\epsilon^{5}}{5 !}[H]^{5} \pm \cdots \\
& \sim \epsilon[H]\left\{1+\frac{1}{3}\left(\frac{\epsilon^{2}}{2 !}[H]^{2}\right)+\frac{1}{5}\left(\frac{\epsilon^{4}}{4 !}[H]^{4}\right)+\cdots\right\} \\
& <\epsilon[H]\left(H_{2 n}\right)< \pm 6[H] e^{\frac{\epsilon}{2}\left[H^{2}\right]} \sim \pm \epsilon[H] .
\end{aligned}
$$

Here, one applies the so-called strong-approach consisting of 2 (two) basic steps as follows : 1. Applying the limit of $H_{2 n}$ and $H_{2 n+1}$ for $\epsilon \rightarrow 0,2$. Applying fieldredefinition after multiplying the exponent- $[H]$ with $\mathrm{VEV}, v$ and $v^{\prime}$ respectively, to obtain new scalar fields from the existing $\widetilde{\Phi}^{(i)^{\prime \prime}}=1,2$ in Eq. (190).

Taking the first step, one finds immediately,

$$
\lim _{\epsilon \rightarrow 0} e^{ \pm \epsilon[H]}=\lim _{\epsilon \rightarrow 0} H_{2 n+1}+\lim _{\epsilon \rightarrow 0} H_{2 n}=1 \pm \epsilon[H] .
$$

Eq. (194) can be approached by the following approximation where $\epsilon=\frac{1}{f}, f^{2}=$ $f_{1}^{2}+f_{2}^{2}, f_{i}, i=1,2 \mathrm{SU}(6) \mathrm{VEVs}$ so that the condition $\epsilon \rightarrow 0$ is achieved. Clearly Eq. (194) can be rewritten as follows,

$$
\left(e^{ \pm \frac{1}{f}[H]}-1\right) \sim \frac{1}{f} H
$$


or, utilising Eq. (??) for $[H]$ and treating by blocks of $3 \times 3$ sub-matrices, can give

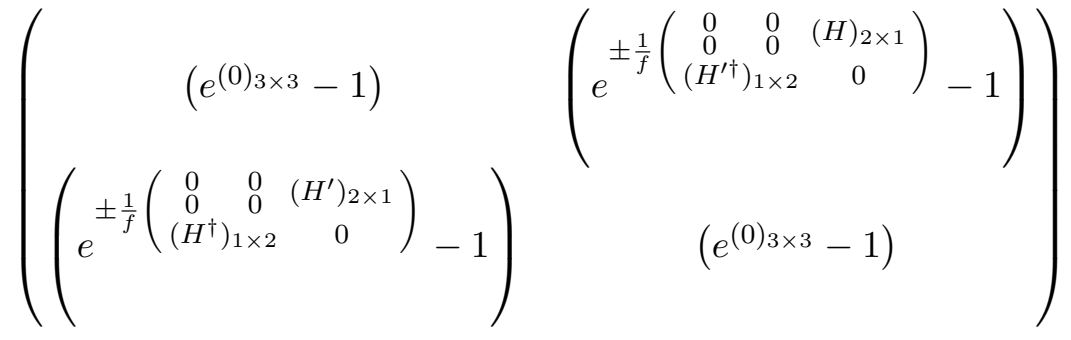

$$
\begin{aligned}
& \sim \frac{1}{f}\left(\begin{array}{ccc}
(0)_{3 \times 3} & \left(\begin{array}{ccc}
0 & 0 & (H)_{2 \times 1} \\
0 & 0 & \\
\left(H^{\prime \dagger}\right)_{1 \times 2} & 0
\end{array}\right) \\
0 & 0 & \left(H^{\prime}\right)_{2 \times 1} \\
\left(H^{\dagger}\right)_{1 \times 2} & 0
\end{array}\right) .
\end{aligned}
$$

Defining $v_{1}=\left(\begin{array}{c}0 \\ 0 \\ f_{1}\end{array}\right)$ and $v_{2}^{\prime}=\left(\begin{array}{c}0 \\ 0 \\ f_{2}\end{array}\right)$ VEVs in Eq. (184) are rewritten as,

$$
v=\left(\begin{array}{c}
v_{1} \\
0
\end{array}\right), \quad v^{\prime}=\left(\begin{array}{c}
0 \\
v_{2}^{\prime}
\end{array}\right)
$$

and LittleHiggs-like scalar $\widetilde{\Phi}^{(i)^{\prime \prime}}=1,2$ can be expanded to the lowest order as,

$$
\begin{aligned}
& \widetilde{\Phi}^{(1)^{\prime \prime}} \sim \\
& \left(\begin{array}{c}
v_{1} \\
0
\end{array}\right)\left\{1-\left(\left(\begin{array}{c}
(0)_{3 \times 3} \\
e^{ \pm \frac{1}{f}\left(\begin{array}{ccc}
0 & 0 \\
0 & 0 & \left(H^{\prime}\right)_{2 \times 1} \\
\left(H^{\dagger}\right)_{1 \times 2} & 0
\end{array}\right)}-1
\end{array}\right)\right\}\right. \\
& \widetilde{\Phi}^{(1)^{\prime \prime}} \sim \\
& \left(\begin{array}{c}
0 \\
v_{2}^{\prime}
\end{array}\right)\left\{1-\left(\left(\begin{array}{c}
(0)_{3 \times 3} \\
e^{ \pm \frac{1}{f}\left(\begin{array}{ccc}
0 & 0 \\
0 & 0 & \left(H^{\prime}\right)_{2 \times 1} \\
\left(H^{\dagger}\right)_{1 \times 2} & 0
\end{array}\right)}-1
\end{array}\right)\right\}\right.
\end{aligned}
$$


After a little calculation one obtains as follows,

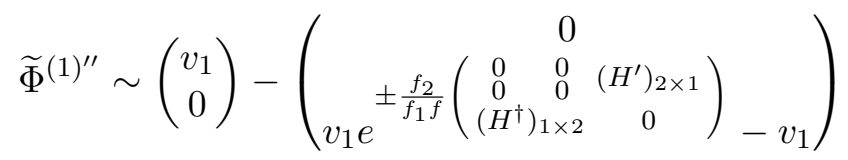

$$
\begin{aligned}
& \widetilde{\Phi}^{(2)^{\prime \prime}} \sim\left(\begin{array}{c}
0 \\
v_{2}^{\prime}
\end{array}\right)-\left(v_{2}^{\prime} e^{ \pm \frac{f_{1}}{f_{2} f}\left(\begin{array}{ccc}
0 & 0 & (H)_{2 \times 1} \\
0 & 0 & \\
\left(H^{\prime \dagger}\right)_{1 \times 2} & 0
\end{array}\right)}-v_{2}^{\prime}\right),
\end{aligned}
$$

where fields $\widetilde{\Phi}^{(i)^{\prime \prime}}, i=1,2$ must be redefined and transformed into new fields $\widetilde{\Phi}^{(i)^{\prime}}, i=$ 1,2 as follows,

$$
\widetilde{\Phi}^{(1)^{\prime}}=\widetilde{\Phi}^{(1)^{\prime \prime}}+\left(\begin{array}{c}
0 \\
v_{1}
\end{array}\right), \quad \widetilde{\Phi}^{(2)^{\prime}}=\widetilde{\Phi}^{(2)^{\prime \prime}}+\left(\begin{array}{c}
v_{2}^{\prime} \\
0
\end{array}\right)
$$

which immediately provide the following results,

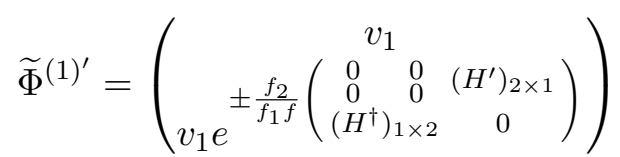

$$
\begin{aligned}
& \widetilde{\Phi}^{(2)^{\prime}}=\left(v_{2}^{\prime} e^{ \pm \frac{f_{1}}{f_{2} f}\left(\begin{array}{ccc}
0 & 0 & (H)_{2 \times 1} \\
0 & 0 & (H)_{2 \times 1} \\
\left(H^{\prime \dagger}\right) 1 \times 2 & 0
\end{array}\right)} v_{2}^{\prime}\right.
\end{aligned}
$$

Rewriting Eq. (202) in the following forms and combining the 6x6 matrices below,

$$
\begin{aligned}
& \widetilde{\Phi}^{(1)^{\prime}}=\left(\begin{array}{ccc}
1 & & 0 \\
\pm \frac{f_{2}}{f_{1} f}\left(\begin{array}{ccc}
0 & 0 & \left(H^{\prime}\right)_{2 \times 1} \\
0 & 0 & \\
\left(H^{\dagger}\right)_{1 \times 2} & 0
\end{array}\right) & 0
\end{array}\right)\left(\begin{array}{c}
v_{1} \\
0
\end{array}\right),
\end{aligned}
$$

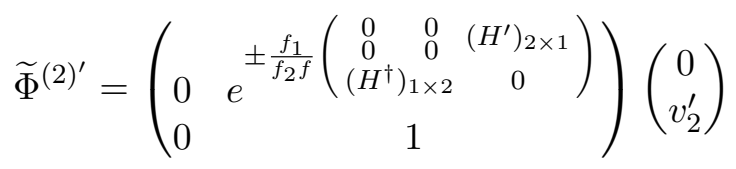

one obtains directly the LittleHiggs-like scalar as,

$$
\begin{aligned}
& \widetilde{\Phi}^{(1)^{\prime}}\left[\widetilde{\Phi}^{(2)^{\prime}}\right]
\end{aligned}
$$

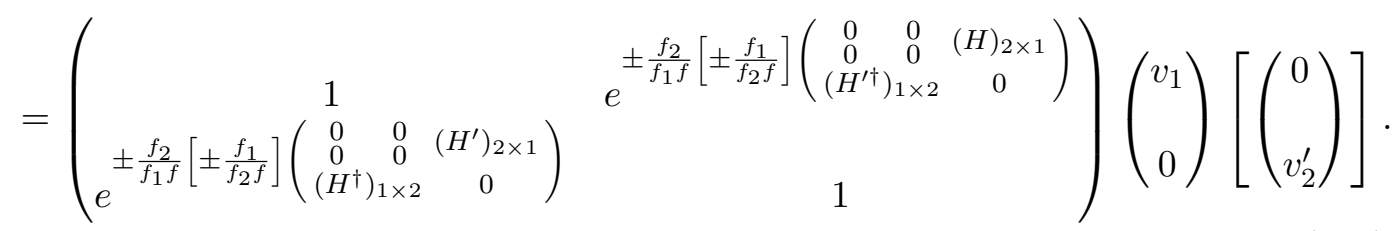

Remembering that $[H]$ in the exponent of $\widetilde{\Phi}^{(i)^{\prime \prime}}, i=1,2$ in Eq.(8) is hermitian one must consequently put factor $\mathrm{i}$ in the exponent of $\widetilde{\Phi}^{(i)^{\prime}}, i=1,2$ in Eq.(23) when it is expressed in general complex form as used in Eq.(1) where $\widetilde{\Phi}^{(i)}, i=1,2$ 
is unitarily-expressed with $h\left(h^{\prime}\right)$ of the parameter- $\theta$ is complex field as shown in Eq.(3). Therefore Eq.(23) is rewritten in unitarily-expressed form as follows,

$$
\begin{aligned}
& \widetilde{\Phi}^{(1)^{\prime}}\left[\widetilde{\Phi}^{(2)^{\prime}}\right]
\end{aligned}
$$

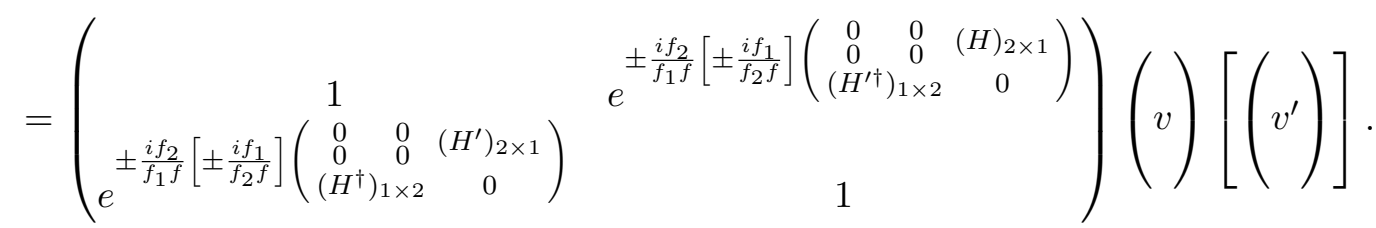

which in direct comparison to the original unitarily-expressed complex form of Eq.(1), as below

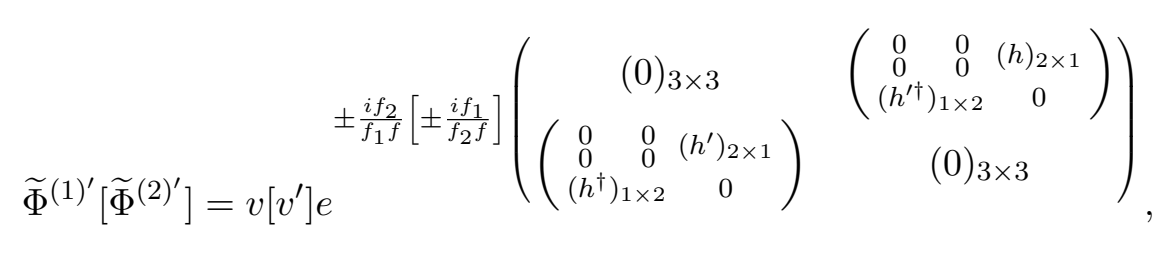

where $|h| \sim|H|$ and $\left|h^{\prime}\right| \sim\left|H^{\prime}\right|$ based on Eq.(3), gives a straightforward relation as shown below.

Here, one must recall the work of J Schwinger [17] where in the strongly-coupled realm the massless boson can not exist with the massive one because the massive boson must be unstable and broken down. Therefore the massless $\widetilde{\Phi}^{(i)}, i=1,2$ and $h$ can only exist and produce a relatively very light bosons $\widetilde{\Phi}^{(i)^{\prime}}, i=1,2$ and $H\left[H^{\prime}\right]$. Considering $\widetilde{\Phi}^{(i)} \sim \widetilde{\Phi}^{(i)^{\prime}}, i=1,2$ in mass the relation above can be expressed as,

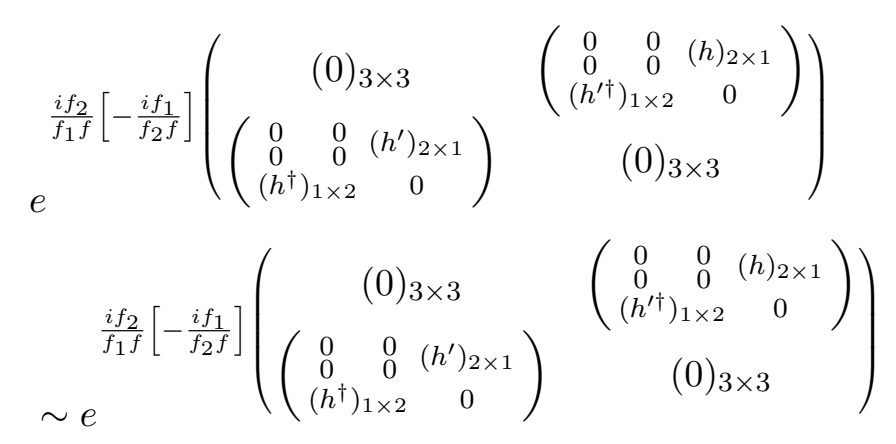

with $|h| \sim|H|$ and $\left|h^{\prime}\right| \sim\left|H^{\prime}\right|$ which is called as a strong approach [3]. This technique is to be applied in the strongly-coupled realm especially for exotic scalars. When it is used to calculate masses from a $\mathrm{SU}(6)$ generalized Coleman-Weinberg potential it gives the same result as given by Baker-Campbell-Hausdorff formula via the twocommutator multiplication which provides later the required quartic potential in $\mathrm{SU}(3)$ triplets as well.

\section{References}

[1] A.Hartanto and L.T.Handoko Phys. Rev. D 71, 095013 (2005). 
[2] A.Hartanto, C.Wijaya, and L.T.Handoko, Jurnal Fizik Malaysia 26, 253 (2005).

[3] A. Hartanto, F. P. Zen, J. S. Kosasih and L. T. Handoko, Near-Brane SU(6)-origin Higgs in Scherk-Schwarz breaking of 5-dimensional SU(6) GUT, IJMPA, Vol. 27, Iss.7, SO217751X12500352, (2012).

[4] A. Hartanto, F. P. Zen, J. S. Kosasih and L. T. Handoko, Proton decay in the 5D SU(6) symmetry breaking via little Higgs and Scherk-Schwarz mechanisms, World Scientific, M. Gell-Mann 80th Birthday Celebration Conference, Singapore, (2010) .

[5] A. Pierce, Dark matter at the LHC, in Perspective on LHC Physics, editor: G-Kane, A.Pierce, World Scientific Publishing Co., Ltd. (2008), Singapore.

[6] C. Amsler et al. (Particle Data Group), Phys. Lett. B 667, 1 (2008).

[7] Carl. E. Wulfman, Dynamical symmetry, World Scientific Publishing Co, Ltd., (2011) Singapore.

[8] C. S. Lim and N. Maru, Towards a realistic grand gauge-Higgs unification, Phys. Lett. B 653320 (2007) [arXiv:hep-ph/07061397].

[9] D. E. Kaplan \& M. Schmaltz, Little Higgs from a Simple Group, JHEP 0310 (2003) 039, [arXiv:hep-ph/0302049].

[10] F. Daniel Steffen, Dark matter candidates (axion, neutralinos, gravitinos, and axinos), (2009), European Physiscs Jounal C (2009) 59:557-558; SpringerVerlag 2008.

[11] G. Burdman and Y. Nomura, Nucl. Phys. B 6563 (2003).

G. Burdman and Y. Nomura, Phys. Rev. D 69115013 (2004).

[12] G. Burdman, Y. Nomura, Unification of Higgs and gauge fields in five dimensions, [arXiv:hep-ph/0210257].

[13] H. C. Cheng, K. T. Matchev, and M. Schmaltz, Phys. Rev. D 66036005 (2002).

[14] H. C. Cheng and I. Low, Journal of high energy physics 09051 (2003).

HC. Cheng, Ian Low, Little hierarchy, little Higgses, and little symmetry, [arXiv:hep-ph/0302049].

[15] Howard E. Haber, Ann E. Nelson, Particle physics and cosmology, TASI 2002, World Scientific Publishing Co. Pte. Ltd, Singapore, (2004).

[16] I.M. Levy-Leblond, Local Heisenberg inequality, Phys. Lett. 111A, 353 (1985).

[17] J. Schwinger, J. McDonald, Gauge singlet scalars as a Cold Dark Matter, Phys. Rev. D50 (1994), 3637 [arXiv:hep-ph/0702143], (2007).

[18] J. Scherk and J. Schwarz, B82 ,60(1979) Phys. Lett. B 8260 (1979). 
[19] J. Scherk and J.Schwarz, Nucl. Phys. B 15361 (1979).

[20] John F. Gunion, HE. Haber, G. Kane, S. Dawson, The Higgs hunter's guide, Addison-Wesley Publishing Co., (1990), USA.

[21] K.R. Dienes, New directions for new dimensions: Kaluza theory, large extra dimension and brane world, TASI 2002: Particle Physics and Cosmology, World Scientific Publishing Co., Ltd., Singapore (2004).

Abdel Perez-Lorenzana, An introduction to extra dimension, Lectures at Mexican School of Particles and Fields, Xalapa, Mexico, August 1-13, (2004).

[22] L.J. Hall, H.Murayama, and Y. Nomura, Nucl. Phys. B 64585 (2002).

L.J. Hall, H. Murayama and Y. Nomura, Wilson lines and symmetry breaking on orbifolds, [arXiv:hep-ph/0707245].

[23] L.J. Hall, Y. Nomura, Gauge coupling unification from unified theories in higher dimension, Phys. Rev D, Vol. 65, 125012, (2002).

[24] M. Kubo, C.S. Lim, H. Yamashita, The Hosotani mechanism in bulk gauge theories with an orbifold extra space $S^{1} / Z_{2}$, [arXiv:hep-ph/0111327], (2002).

[25] M. Muck, A. Pilaftsis, R. Ruek Minimal higher-dimensional extensions of the standard model and electroweak observables, (2001) [arXiv:hep-ph/0110391].

[26] M. Quiros, New ideas in symmetry breaking, TASI 2004, Physics in D $\geq 4$, World Scientific Publishing Co., Singapore, (2006).

[27] M. Schmaltz, The Simplest Little Higgs, JHEP 0408 (2004) 056, [arXiv:hep$\mathrm{ph} / 0407143]$.

[28] M. Schmaltz, Physics beyond the SM theory: Introducing the little Higgs, Nucl. Phys. Proc. Suppl. 117 (2003) 40, [arXiv:hep-ph/0210415].

[29] M. Schmaltz and D. Tucker-Smith, Annual review of nuclear and particle science $\mathbf{5 5} 229$ (2005).

M. Schmaltz and D. Tucker-Smith, Little Higgs review, [arXiv:hep$\mathrm{ph} / 0502182]$.

[30] M. Razavy, Heisenberg's Quantum Mechanics, World Scientific Publishing Co., Ltd. (2011), Singapore.

[31] N. Arkani-Hamed, A. G. Cohen and H. Georgi, Electroweak symmetry breaking from dimensional deconstruction, Phys. Lett. B513, 232 (2001), [arXiv:hep$\mathrm{ph} / 0105239]$.

[32] N. Arkani-Hamed, A. G. Cohen, E. Katz, A. E. Nelson, T. Gregoire and J. G. Wacker, JHEP 0208, 021 (2002), [arXiv:hep-ph/0206020].

[33] N. Arkani-Hamed, A. G. Cohen, E. Katz and A. E. Nelson, The little Higgs, JHEP 0207, 034 (2002), [hep-ph/0206021]. 
[34] N. Arkani-Hamed, M. Schmaltz, Phys. Rev. D 61033005 (2000).

[35] P. Ramond, Journeys beyond the standard model, Persus Books, Cambridge, Massachusetts, (1999), USA.

[36] R. Contino, Y. Nomura, and A. Pomarol, Nucl. Phys. B 671148 (2003).

[37] S. Chang and J. G. Wacker, Little Higgs and custodial SU(2), [arXiv:hep$\mathrm{ph} / 0303001]$.

[38] S. Chang, A little Higgs model with custodial SU(2), [arXiv:hep-ph/0306034].

[39] S. Oneda, Y. Koide, Asymptotic symmetry and its implication in elementary particle physics, World Scientific Publishing Co., Ltd. (1991), Singapore.

[40] S. Oneda, H. Umezawa, S. Matsuda, Phys. Lett. 25, 71 (1970).

S. Oneda, H. Umezawa, S. Matsuda, Phys. Rev. D2, 324 (1970).

[41] S.R. Coleman and E. Weinberg, Phys. Rev. D 71888 1973).

[42] T. Mori, C. S. Lim and S. N. Mukherjee, The physics of the standard model and beyond, World Scientific Publishing Co. Pte. Ltd, Singapore, (2001).

[43] W.G. Faris, I.M. Levy-Leblond, Correlation of quantum theory properties and the generalized Heisenberg inequality, Am. I. Phys. 54, 135 (1986)

[44] W.Greiner, B. Muller, Gauge theory of weak interaction, Springer-Verlag, Berlin (1993) Willi-Hans Steeb, Problems and Solution in Theoretical and Mathematical Physics, World Scientific Publishing Co. Pte. Ltd, Singapore, (2003).

[45] W. Skiba and J. Terning, A simple model of two little Higgses, Phys. Rev. D68 (2003) 075001, [arXiv:hep-ph/0305302].

[46] Y. Hosotani, Phys. Lett. B 126 309) (1983).

[47] Y. Kawamura, Triplet-doublet splitting, proton stability and an extra dimension, [arXiv:hep-ph/0012125], (2001).

\section{Received: January 15, 2014}

